$$
\text { DOEPU/NE-02-06 }
$$

PU/NE-02-06

DOE Final Report

\title{
INTERFACIAL AREA AND INTERFACIAL TRANSFER \\ IN TWO-PHASE FLOW SYSTEMS
}

by

M. Ishil, T. Hibiki, S. T. Revankar, S. Kim, and J. M. Le Corre

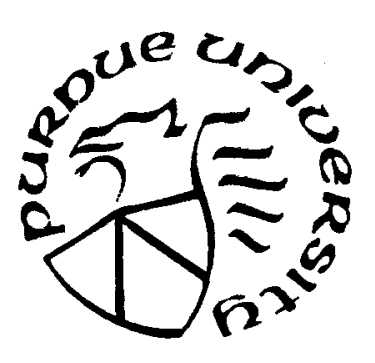

DOE Patent Clearance Granted

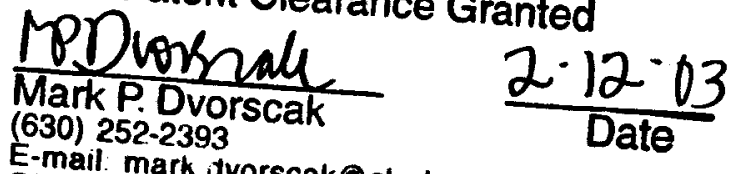

Office of Imrkellectual Property.doe.gov

DOE Chicago Operationerty Law

\section{PURDUE UNIVERSITY}

SCHOOL OF NUCLEAR ENGINEERING 


\section{DISCLAIMER}

This report was prepared as an account of work sponsored by an agency of the United States Government. Neither the United States Government nor any agency thereof, nor any of their employees, makes any warranty, express or implied, or assumes any legal liability or responsibility for the accuracy, completeness, or usefulness of any information, apparatus, product, or process disclosed, or represents that its use would not infringe privately owned rights. Reference berein to any specific commercial product, process, or service by trade name, trademark, manufacturer, or otherwise does not necessarily constitute or imply its endorsement, recommendation, or favoring by the United States Government or any agency thereof. The views and opinions of authors expressed herein do not necessarily state or reflect those of the United States Government or any agency thereof. 


\section{DISCLAIMER}

Portions of this document may be illegible in electronic image products. Images are produced from the best available original document. 
PU/NE-02-06

PURDUE UNIVERSITY

SCHOOL OF NUCLEAR ENGINEERING

INTERFACIAL AREA AND INTERFACIAL TRANSFER

IN TWO-PHASE FLOW SYSTEMS

by

M. Ishii, T. Hibiki*, S. T. Revankar, S. Kim, J. M. Le Corre

School of Nuclear Engineering, Purdue University, USA**

July 2002

Work supported by

U.S. Department of Energy, Office of Basic Energy Science

* Visiting Associate Professor of Purdue University, Present address: Research Reactor Institute, Kyoto University, Kumatori, Sennan, Osaka 590-0494, Japan

** 1290 School of Nuclear Engineering, West Lafayette, IN 47907-1290, USA 


\section{ACKNOWLEDEMENTS}

The authors would like to express their sincere appreciation to Dr. W. H. Leung, Prof. Q. Wu, Dr. Y. Mi, Dr. X. Y. Fu, Mr. A. Kashyap, Mr. S. Hogsett, Mr. Z. Xiao, Mr. F. Takada, and Mr. X. Wang for their help in performing this project. The authors would also like to express their special thanks to Professor Delhaye (CEA, Grenoble, France) for his fruitful discussions. The assistance provided by the members of the laboratory and research group is also much appreciated. The authors would also like to extend their gratitude to the staff of the School of Nuclear Engineering and the Machine Shop at the airport laboratory for their assistance.

The research project was performed under the auspices of the US Department of Energy's Office of Basic Energy Science. The authors would like to express their sincere appreciation for the encouragement, support and technical comments on this program from Drs. Manley, Goulard, and Price of DOE/BES. 


\section{TABLE OF CONTENTS}

Page

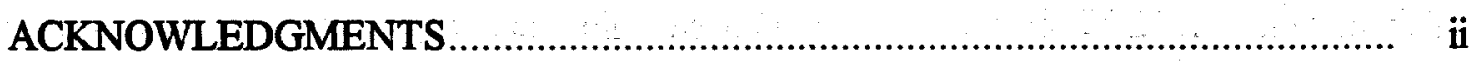

TABLE OF CONTENTS ........................................................................... iii

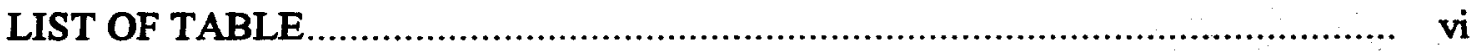

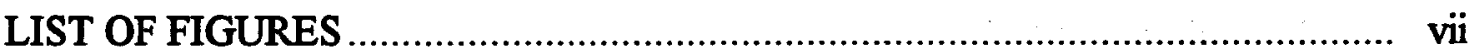

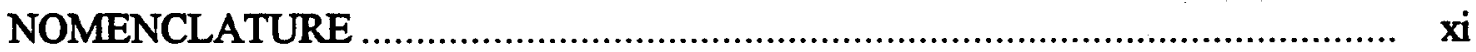

ABSTRACT

1. INTRODUCTION

2. MEASUREMENT TECHNIQUES..............................................................

2.1. Multi Sensor Probe Methodology ……................................................... 7

2.1.1. Measurement Principle .................................................................. 7

2.1.2. Development of the Miniaturized Conductivity Probe and the Signal Processing Scheme.................................................................. 9

2.1.2.1. Design of the Miniaturized Four-Sensor Probe................................ 10

2.1.2.2. Signal Processing Scheme ......................................................... 12

2.1.3. Benchmark Experiment .......................................................................... 19

2.1.4. Benchmark of the New Four-Sensor Conductivity Probe ...................... 24

2.1.4.1. Benchmark of the Double-Sensor Conductivity Probe....................... 24 
2.1.4.2. Benchmark of the Four-Sensor Conductivity Probe.

2.1.5. Conductivity and Optical Four-Sensor Probe Benchmark.

2.1.6. Numerical Evaluation and Correction Method for Multi-Sensor Probe Measurement Techniques

2.2. Hotfilm Anemometry Methodology

3. TWO-GROUP INTERFACIAL AREA TRANSPORT EQUATIONS

3.1. Fluid Particle Number Density Transport Equation .

3.2. Fluid Particle Interfacial Area Concentration Transport Equation.

4.1. Classification of Interfacial Area Transport Mechanisms Due to Bubble Coalescence and Breakup 46

4.2. Random Collision Induced Bubble Coalescence

4.3. Wake-entrainment Induced Bubble Coalescence 50

4.4. Bubble Breakup Due to Turbulent Impact.

4.5. Final Equations of Source and Sink Terms in Two-group Interfacial Area

Transport Equations

5. DESULTS AND DISCUSSION.

5.1. Data Base Used for Evaluation of the Derived Model

5.2. Predictions of Flow Parameters Used in Interfacial Area Transport Calculation. 66

5.3. Contributions of Bubble Coalescence, Breakup and Expansion to One-group Interfacial Area Transport

5.4. Contributions of Bubble Coalescence, Breakup and Expansion to Two-group Interfacial Area Transport 
LIST OF JOURNAL PUBLICATIONS RELATED TO DEVELOPMENT OF INTERFACIAL AREA TRANSPORT EQUATION PERFOMRED AT THERMAL-HYDRAULICS AND REACTOR SAFETY LABORATORY IN PURDUE UNIVERISTY. 


\section{LIST OF TABLES}

Table

Page

4.1 Sink and source interactions of bubbles for bubble number density. .............. 48

4.2 Summary of the equations to predict the interracial area concentration............ 58

4.3 Summary of the equations to predict the void fraction............................... 59

4.4 List of measured quantities and unknown quantities to be calculated.............. 59

5.1 Dimensions of flow loop used in the experiment................................... 60

5.2 Flow conditions in the experiment using a $50.8 \mathrm{~mm}$-diameter pipe............... 61

5.3 Empirical correlations used in the predictions of system pressure and

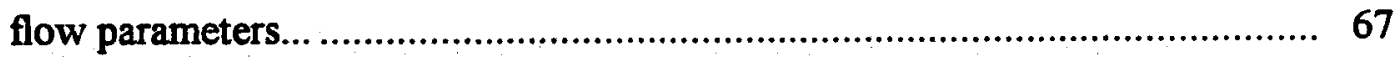

5.4 Typical initial values used in the interfacial area transport calculation. ............. 67

5.5 Summary of the adjustable valuables to predict the interfacial area

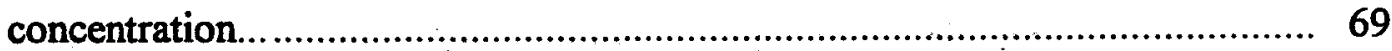




\section{LIST OF FIGURES}

Figure

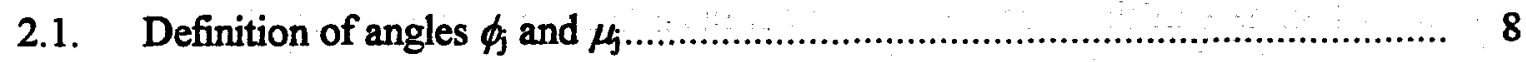

2.2. Photographic image of the probe sensors with/without dielectric coating......... 10

2.3. Schematic diagrams of the conventional and new four-sensor conductivity probes.

2.4. The signals before and after the signal conditioning processes.

2.5. Examples of defective signals.

2.6. Schematic diagram of the experimental loop designed for the benchmark experiment of the newly-designed miniaturized four-sensor conductivity probe

2.7. The characteristic experimental results obtained by the four-sensor conductivity probe at $z / D=32$ with $\left\langle j_{B}\right\rangle=0.432 \mathrm{~m} / \mathrm{s}$ and $\left\langle j_{\mathrm{P}}\right\rangle=0.321 \mathrm{~m} / \mathrm{s}$. The time-averaged local (a) void fraction and (b) interfacial area concentration of group I and group II bubbles, and (c) for Taylor bubbles

2.8. Local time-averaged void fraction and interfacial area concentration in three different flow conditions for (a) \& (b) for group I and (c) \& (d) group II bubbles; $\left\langle j_{\mathrm{P}}\right\rangle=0.321 \mathrm{~m} / \mathrm{s} \&\left\langle j_{\mathrm{g}}\right\rangle$ varied

2.9. Development of void fraction and Sauter mean diameter along the axial direction of the flow duct. Solid data points measured at $z / D=32$ and blank data points measured at $z / D=64 .\left\langle j_{\mathrm{p}}\right\rangle=0.321 \mathrm{~m} / \mathrm{s}$ and $\left.(\mathrm{a})<j_{\mathrm{g}}\right\rangle=0.052 \mathrm{~m} / \mathrm{s}$, 
(b) $\left\langle j_{\mathrm{B}}>=0.179 \mathrm{~m} / \mathrm{s}\right.$. (c) $\left\langle j_{\mathrm{g}}\right\rangle=0.432 \mathrm{~m} / \mathrm{s}$, and (d) Sauter mean diameters of group I bubbles

2.10. Development of two-phase parameters for Taylor bubbles in three different flow conditions. Solid data points at $z / D=32$ and blank data points at $z / D=64$.

2.11. Photographic image captured for image analysis in benchmarking the double -sensor conductivity probe and its coordinate system. $\left\langle j_{g}\right\rangle=0.023 \mathrm{~m} / \mathrm{s}$ and $\left\langle j_{\mathrm{p}}\right\rangle=0.315 \mathrm{~m} / \mathrm{s}$

2.12. Typical results obtained from the comparison between the interfacial area concentration measured by the double-sensor probe and that from image analysis. Here, $W=$ half-width of the total flow duct width in the $x$-direction.

2.13. Examples of Taylor bubble images captured by $\mathrm{CCD}$ camera. The frame size shown here; width $=6.35 \mathrm{~cm}$ and length $=14.55 \mathrm{~cm}$.

2.14. Comparison of the void fraction and interfacial area concentration between the experimental data and the values calculated based on the image analysis for flow conditions; $\left\langle j_{\mathrm{P}}\right\rangle$ fixed at $0.321 \mathrm{~m} / \mathrm{s}$ and $\left\langle j_{\mathrm{g}}\right\rangle$ varied at (a) $\left\langle j_{\mathrm{g}}\right\rangle=0.052 \mathrm{~m} / \mathrm{s}$, (b) $\left\langle j_{g}>=0.179 \mathrm{~m} / \mathrm{s}\right.$, and $\left.(\mathrm{c})<j_{\mathrm{g}}\right\rangle=0.432 \mathrm{~m} / \mathrm{s}$.

2.15. Comparison between the area-averaged values of measured and calculated void fraction and interfacial area. Error bars shown here $\pm 10 \% \ldots \ldots \ldots \ldots \ldots \ldots \ldots . .29$

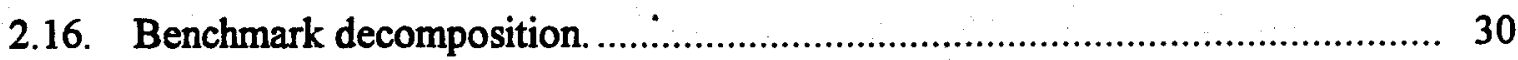

2.17. System component impacts on two-phase flow parameter measurements......... 31

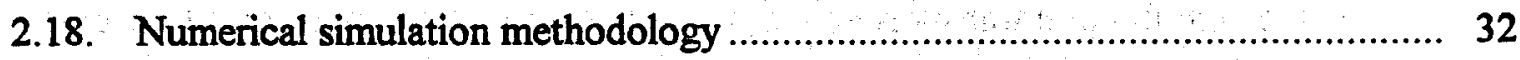

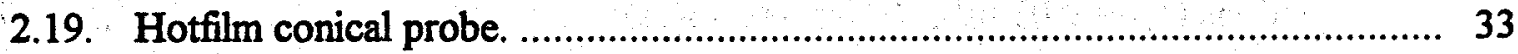

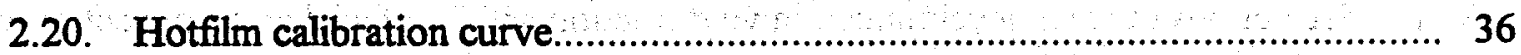

2.21. Raw and filtered hotfilm probe signal ................................................. 36

2.22. Verification of filter used in hotfilm anemometry by void fraction data. ........... 38

4.1. Classification of possible interactions of two-group bubbles........................ 47

4.2. Schematic diagram of wake entrainment model......................................... 52

5.1. Flow conditions in the experiment using a $50.8 \mathrm{~mm}$ diameter pipe................... 61

5.2. Axial development of one-dimensional void fraction, interfacial area concentration, 
and Sauter mean diameter of small and cap bubbles.

5.3. Comparison of measured interfacial area concentration change along the flow direction with one predicted by the one-group interfacial area transport equation $\left.(<j \mathrm{p}\rangle=0.491 \mathrm{~m} / \mathrm{s},\left\langle j_{\mathrm{g}, 0}\right\rangle=0.0275 \mathrm{~m} / \mathrm{s}\right)$.

5.4. Comparison of measured interfacial area concentration change along the flow direction with one predicted by the one-group interfacial area transport equation $\left(\langle j \mathrm{f}\rangle=0.491 \mathrm{~m} / \mathrm{s},\left\langle j_{\mathrm{g}, 0}\right\rangle=0.190 \mathrm{~m} / \mathrm{s}\right)$.

5.5. Examples of axial change of system pressure, void fraction, and Sauter mean diameter.

5.6. Prediction of axial change of one-dimensional interfacial area concentrations measured in a $50.8 \mathrm{~mm}$ diameter pipe by one-group (solid lines) and two-group (broken lines) interfacial area transport equations with modeled sink and source terms

5.7. Comparison between measured and predicted interfacial area concentrations... 74

5.8. Comparison between one-group interfacial area transport equation and data taken by Grossetete (1995).

5.9. Predictions of axial development in void fraction and interfacial area concentration by the void fraction transport equation and two-group interfacial area transport equations $\left(\langle j \mathrm{p}\rangle=0.986 \mathrm{~m} / \mathrm{s},\left\langle j_{g}, 0=0.321 \mathrm{~m} / \mathrm{s}\right)\right.$

5.10. Predictions of axial development in void fraction and interfacial area concentration by the void fraction transport equation and two-group interfacial area transport equations $\left(\left\langle j_{\mathrm{f}}>=2.01 \mathrm{~m} / \mathrm{s},\left\langle j_{\mathrm{g}, 0}>=0.624 \mathrm{~m} / \mathrm{s}\right)\right.\right.$.

5.11. Predictions of axial development in void fraction and interfacial area concentration by the void fraction transport equation and two-group interfacial area transport equations $\left(\langle j \mathrm{j}\rangle=5.00 \mathrm{~m} / \mathrm{s},\left\langle j_{\mathrm{g}, 0}\right\rangle=1.11 \mathrm{~m} / \mathrm{s}\right)$

5.12. Predictions of axial development in void fraction and interfacial area concentration by the void fraction transport equation and two-group interfacial area transport equations $\left(\left\langle j \mathrm{p}>=5.00 \mathrm{~m} / \mathrm{s},\left\langle j_{g, 0}>=3.90 \mathrm{~m} / \mathrm{s}\right)\right.\right.$

5.13. Comparison between measured and predicted void fractions 
5.14. Scale analysis to determine the predominant terms among the source and sink terms .

5.15. Sensitivity analysis of adjustable valuables in the two-group interfacial area transport equations 85

A.1. Order of magnitude of the frequency of bubble random collision 92 


\section{NOMENCLATURE}

A frontal area of the bubble

$A_{\mathrm{i}, \mathrm{k}} \quad$ average interfacial area of the $k$-group particles of volume $v$

$A_{\mathrm{s}} \quad$ measurement area formed by the double sensors

$A_{0} \quad$ pressure gradient

$a_{i} \quad$ interfacial area concentration

$C_{\mathrm{D}} \quad$ drag coefficient

c ratio of the minimum eddy size, which would not cause bubble breakup, to the bubble diameter

$D \quad$ pipe diameter

$D_{\mathrm{b}} \quad$ bubble diameter

$D_{\mathrm{C}} \quad$ chord length of cap bubble

$D_{\text {Cmax }} \quad$ maximum cap bubble limit

$D_{\text {dmas }} \quad$ maximum distorted bubble limit

$D_{\mathrm{ds}} \quad$ maximum spherical bubble limit

$D_{c} \quad$ eddy diameter

$D_{\text {eq.H-C }}$ equivalent bubble diameter given by Chester and Hoffman

$D_{\mathrm{Sm}} \quad$ Sauter mean diameter

Eo Eötvös number

$f \quad$ collision frequency

$f(\vec{x}, v, t)$ particle density distribution function

$j \quad$ superficial velocity

K coefficient 


\begin{tabular}{|c|c|}
\hline$K^{\circ}$ & coefficient \\
\hline$L$ & pipe length \\
\hline$L_{\mathrm{b}}$ & average distance between bubbles \\
\hline$L_{\mathrm{WE}}$ & $\begin{array}{l}\text { critical distance at which the leading bubble began to exert a noticeable influence } \\
\text { on the following one }\end{array}$ \\
\hline$l_{\mathrm{s}}$ & separation distance between the probe sensors \\
\hline$N_{\mathrm{b}}$ & number of bubbles passing the probe per unit time \\
\hline$N_{\text {WE }}$ & number of small bubbles inside the effective wake region \\
\hline$N_{\mu f}$ & liquid viscosity number \\
\hline$n$ & unit normal vector \\
\hline$n$ & number density of particles of all sizes \\
\hline$n_{\mathrm{e}}$ & number of eddies of wave number per volume of two-phase mixture \\
\hline$P$ & pressure \\
\hline$R$ & pipe radius \\
\hline$r$ & radial position \\
\hline $\operatorname{Re}$ & Reynolds number \\
\hline$S_{\mathrm{j}}$ & $\begin{array}{l}\text { net rate of change in the number density function due to the particle breakup and } \\
\text { coalescence processes integrated from possible minimum particle volume to } \\
\text { possible maximum particle volume }\end{array}$ \\
\hline$S_{\text {ph }}$ & $\begin{array}{l}\text { net rate of change in the number density function due to the phase change } \\
\text { Integrated from possible minimum particle volume to possible maximum } \\
\text { particle volume }\end{array}$ \\
\hline$s_{\mathrm{j}}$ & $\begin{array}{l}\text { net rate of change in the number density function due to the particle breakup and } \\
\text { coalescence processes }\end{array}$ \\
\hline$s_{\mathrm{ph}}$ & fluid particle sink or source rate due to the phase change \\
\hline$T$ & total time \\
\hline$t$ & time $\quad$ \\
\hline$U_{\infty}$ & relative velocity between gas and liquid phases \\
\hline$V$ & voltage \\
\hline
\end{tabular}


xiii

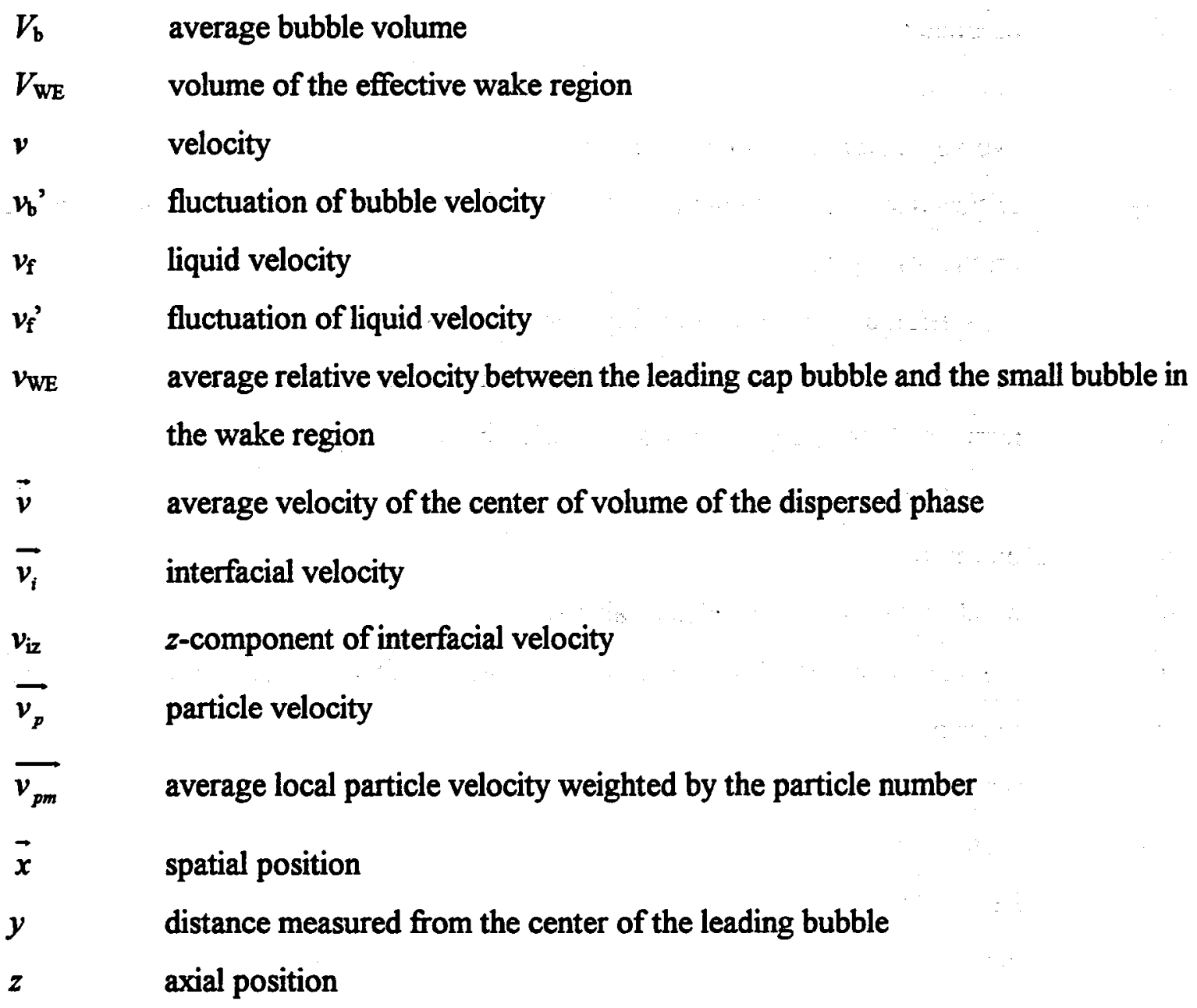

Greek letters

$\alpha \quad$ void fraction

$\alpha_{\max } \quad$ maximum allowable void fraction

$\alpha_{0} \quad$ maximum angle possible in $\mu_{\mathrm{j}}$

$\beta \quad$ ratio of the mixing length and the width of the wake

$\Gamma \quad$ adjustable parameter

$\gamma \quad$ adjustable parameter

$\Delta T$ average time interval required for collision of all bubbles in the wake region with the leading cap bubble

$\Delta \rho \quad$ density difference

$\varepsilon \quad$ energy dissipation 
$\varepsilon_{0} \quad$ energy dissipation obtained from the mechanical energy equation

$\zeta \quad$ distribution function to specify the rate of the collision between turbulent eddies and bubbles producing small daughter bubbles to total collision

$\boldsymbol{\kappa}$ coefficient

$\Lambda$

factor

$\lambda$ breakup or coalescence efficiency

$\rho$

density

$\sigma \quad$ interfacial tension

$\sigma_{\mathrm{z}} \quad$ velocity fluctuation

$v \quad$ volume of a particle

$U_{\max } \quad$ possible maximum particle volume

$v_{\min } \quad$ possible minimum particle volume

$\Phi_{\mathrm{j}}$ rate of change of interfacial area concentration due to bubble breakup or coalescence, $\left(2 a_{\mathrm{i}} / 3 \alpha\right) \phi_{\mathrm{j}}$

$\phi_{j}$ angle between the bubble interfacial velocity and the unit normal vector of the $j$-th interface

$\mu_{j} \quad$ angle between the probe sensor and the unit normal vector of the $j^{\text {th }}$ bubble interface

$\Psi \quad$ rate of change of void fraction due to bubble breakup or coalescence

$\psi \quad$ factor depending on the shape of the bubbles

$\Omega \quad$ sensitivity

\section{Subscripts}

$0 \quad$ value at the inlet of the test section

1 bubbles belonging to group I

12 interchange term

2 bubbles belonging to group II

21 interchange term

b bubble 
calc. calculated value

eff effetive

F friction

f liquid phase or front bubble interface

g gas phase

i interface

k bubbles belonging to group $\mathbf{k}$

m two-phase mixture

meas. measured value

min minimum

miss missing bubble interface

$\max$ maximum

norm normalized

RC random collision

$r$ rear bubble interface

TI turbulent impact

tot total

WE wake entrainment

Symbols

$<>\quad$ cross-sectional area averaged quantity

$<<>\quad$ void fraction weighted cross-sectional area averaged quantity

$<<>$ interfacial area concentration weighted cross-sectional area averaged quantity 


\begin{abstract}
In relation to the development of the interfacial area transport equation in two-phase flow systems, the development of local flow measurement techniques, the formulation of two-group interfacial area transport equations, the construction of database, the modeling of sink and source terms of interfacial area concentration were performed.

As for the development of local flow measurement technique for gas phase, a miniaturized four-sensor conductivity probe and its signal processing scheme were developed. The sharp and highly conductive sensor tips, and miniaturized structure of the probe configuration could effectively minimize bubble deformation and missing bubble phenomena. Moreover, the new design accommodated the capability of a double-sensor probe for small bubbles, such that the two-phase parameters for both small and large bubbles could be obtained simultaneously. This was a significant improvement in view of building a database for the development of the two-group interfacial transport equation, because the new probe could be applied in flow conditions where different types of bubbles existed simultaneously. The four-sensor conductivity probe method was benchmarked by a well-designed experiment and a numerical simulation, its validity was demonstrated analytically and experimentally. The four-sensor conductivity probe system developed in this study at Purdue University was compared with the four-sensor optical probe system developed by French Atomic Energy Commission, France. It gave us some very useful information on the capabilities of both systems as well as on the main system components influencing the measurement accuracy.
\end{abstract}

As for the development of local flow measurement technique for liquid phase, hotfilm anemometer developed basically for single-phase flow was applied to two-phase 
flow. In order to apply the hotfilm anemometer to two-phase flow, a signal processing scheme was developed to remove signals due to passing bubbles from raw signals. The hotfilm probe method was benchmarked by a well-designed experiment and its validity was demonstrated experimentally.

As for the construction of database of local flow parameters, extensive experiments were performed basically by using $25.4-\mathrm{mm}$ and $50.8-\mathrm{mm}$ round tubes. The data from the multi-sensor conductivity probe gave near complete information on the time-averaged local hydrodynamic parameters of two-phase flow to model and evaluate sink and source terms of interfacial area concentration. Thus, the data set obtained in this study will eventually be used for the development of reliable constitutive relations, which reflect the true transfer mechanisms in bubbly flow systems.

As for the formulation of two-group interfacial area transport equations, a general approach to treat bubbles in two groups was proposed. The two-group interfacial area transport equations were formulated by considering two-groups of bubbles such as spherical/distorted bubbles (Group I) and cap/slug bubbles (Group II). Possible bubble coalescence and breakup patterns were classified into eight categories including the interchange terms between groups I and II.

As for the modeling of source and sink terms in the interfacial area transport equation, four mechanisms of bubble coalescence and breakup were considered: (1) the coalescence due to random collisions driven by turbulence, (2) the coalescence due to wake entrainment, (3) the breakup upon the impact of turbulent eddies, and (4) the breakup of large cap bubbles due to flow instability on the bubble surface. Based on the four basic mechanisms, the source and sink terms in the interfacial area transport equations were modeled. The one-group interfacial area transport equation could be applied to flow conditions for which the void fractions were lower than about $20 \%$. The mechanisms of bubble coalescence and breakup were successfully modeled by considering bubble random collision and impact of turbulent eddies. The interfacial area transport calculation by derived models suggested that the bubble interactions such as coalescence and breakup might not contribute to the interfacial area transport for relatively low liquid velocity and void fraction 
because of small bubble mean free path and breakup efficiency. As a consequence, the bubble expansion due to pressure reduction along the flow direction was dominant for the interfacial area transport. On the other hand, as the void fraction was increased, the contribution of the bubble random collision came to be significant for the interfacial area transport.

The two-group interfacial area transport equations were introduced to explain the interfacial area transport phenomena at the bubbly-to-slug flow transition. The preliminary model development for the bubble coalescence and breakup was conducted by considering bubble random collision, wake entrainment and turbulent impact. It turned out that the interchange term due to the turbulent impact on cap bubbles (turbulent impact) and the sink term in the group II due to the wake entrainment of cap bubbles played a minor role in the interfacial area transport in the tested flow conditions. This was explained by considering the small number of turbulent eddies available for the collision with cap bubbles, and the average distance between cap bubbles being much larger than the wake length effective for acceleration of cap bubbles.

The one-group interfacial area transport equation could predict interfacial area concentrations measured in a relatively low void fraction region within an average relative deviation of $\pm 9.51 \%$. The two-group interfacial area transport equations could give good predictions for the interfacial area concentration measured at the bubbly-to-slug flow transition within the average relative deviation of $\pm 3.61 \%$. It was demonstrated that the two-group interfacial area transport equations were promising for the prediction of the interfacial area transport in two-phase flow systems. 


\section{INTRODUCTION}

In the past twenty-five years, significant developments in the two-phase flow formulation have been accomplished by the introduction of the drift flux model and the two-fluid model. In the present state-of-the-art, the two-fluid model can be considered the most detailed and accurate macroscopic formulation of the thermo-fluid dynamics of two-phase systems. In the two-fluid model, the field equations are expressed by the six conservation equations consisting of mass, momentum and energy equations for each phase. Since these field equations are obtained from an appropriate averaging of local instantaneous balance equations, the phasic interaction terms appear in each of the averaged balance equation. These terms represent the mass, momentum and energy transfers through the interface between the phases. The existence of the interfacial transfer terms is one of the most important characteristics of the two-fluid model formulation. These terms determine the rate of phase changes and the degree of mechanical and thermal non-equilibrium between phases, thus they are the essential closure relations which should be modeled accurately. However, the closure relations for these interfacial transfer terms are the weakest link in the two-fluid model at present. The interfacial transfer terms are strongly related to the interfacial area concentration and to the local transfer mechanisms such as the degree of turbulence near interfaces. Basically, the interfacial transport of mass, momentum and energy is proportional to the interfacial area concentration, $a_{i}$, and to a driving force. The interfacial area concentration, defined as the interfacial area per unit volume of the mixture, characterizes the kinematic effects; therefore, it must be related to the structure of the two-phase flow field. On the other hand, the driving forces for the interphase transport characterize the local transport mechanism, and they must be modeled separately (Kocamustafaogullari and Ishii, 1995). Among them, this study will focus on the development of a closure relation for the interfacial area concentration. The brief summary will be given below for several problems of the current closure relation for the interfacial area concentration and a new concept to overcome the problems.

In the present system codes like RELAP5, TRAC and CATHARE, the interfacial area concentration is given by empirical correlations. The correlations are based on two-phase flow regimes and regime transition criteria that do not dynamically represent the 
changes in interfacial structure (Uhle et al., 1998). Ishii et al. (1998) pointed out the following shortcomings caused by the static approach.

(1) The flow regime transition criteria are algebraic relations for steady-state, fully developed flows. They do not fully reflect the true dynamic nature of changes in the interfacial structure. Hence, the effects of the entrance and developing flow can not be taken into account correctly, nor the gradual transition between regimes.

(2) The method based on the flow regime transition criteria is a two-step method, which requires flow configuration transition criteria and interfacial area correlations for each flow configuration. The compound errors from the transition criteria and area correlations can be very significant.

(3) The transition criteria and flow regime dependent interfacial correlations are valid in limited parameter ranges for certain specific operational conditions and geometries. Most of them are obtained from simple experiments and phenomenological models. Often the scale effects of geometry and fluid properties are not correctly taken into account. When applied to high-to-low pressure steam-water transients, these models may cause significant discrepancies, artificial discontinuities and numerical instability.

Recently, the introduction of the interfacial area transport equation has been recommended to solve such problems (Ishii, 1975; 1997a; 1997b; Ishii et al., 1998; Uhle et al., 1998; Wu et al., 1998a). The interfacial area transport equation can be obtained by considering the fluid particle number density transport equation analogous to Boltzmann's transport equation (Kocamustafaogullari and Ishii, 1995). It can replace the traditional flow regime maps and regime transition criteria. The changes in the two-phase flow structure are predicted mechanistically by introducing the interfacial area transport equation. The effects of the boundary conditions and flow development are efficiently modeled by this transport equation. Such a capability does not exist in the current state-of-the-art. Thus a successful development of the interfacial area transport equation can make a quantum improvement in the two-fluid model formulation. The effective use of a similar approach was demonstrated for the analysis of a choked flow by Riznic and Ishii (1989). They used the bubble number density transport equation, wall nucleation source based on the nucleation site density model and bubble expansion model, which had a memory of where bubbles were generated.

The transport equation requires several closure relations to model the fluid 
particle coalescence and disintegration. One would need also closure relations for the interfacial stretching (e.g. bubble oscillations), changes in interfacial shapes due to phase changes or density changes and appearance or disappearance of nuclei. The development of the sink and source terms in the transport equation heavily depends on understanding the mechanisms of particle coalescence and disintegration as well as accurate experimental data for the changes in the interfacial area in two-phase flow. Some phenomenological models for the sink and source terms have been proposed based on the mechanism for fluid particle coalescence and breakup (Coulaloglou and Tavlarides, 1977, Prince and Blanch, 1990, Lafi and Reyes, 1991; Tsouris and Tavlarides, 1994). The general approach to treat the bubbles in two groups was recently proposed by Ishii and coworkers (Ishii et al., 1998; Wu et al., 1998a, Uhle et al., 1998). These two groups: the spherical/distorted bubble group and the cap/slug bubble group would result in two bubble number density transport equations that involve the inner and inter group interactions. Ishii et al. summarized the mechanisms of these interactions in five categories: the coalescence due to random collisions driven by turbulence, the coalescence due to wake entrainment, the breakup due to the impact of turbulent eddies, the shearing-off of small bubbles from cap bubbles, and the breakup of large cap bubbles due to flow instability on the bubble surface. They reduced the two-group transport equations to one-group for a bubbly flow with relatively low void fraction where the bubble size distribution was almost uniform, and developed the model of the sink and source terms (Ishii et al., 1997; Wu et al.,; 1997a; 1998b). The obtained source and sink terms were evaluated by some existing data of bubbly flow in a low void fraction region $(<10 \%)$, which were taken by Kashyap et al. (1994). Although sink and source terms due to bubble coalescence and breakup were appropriately modeled, the gas phase was assumed to be incompressible in their analysis (Ishii et al., 1997; Wu et al, 1997a; 1998b). In other words, the one-group interfacial area transport equation derived under the assumption of incompressible flow, namely constant void fraction along the axial direction was unfortunately utilized in the evaluation of the derived sink and source terms. It turned out from recent studies by the present authors (Hibiki and Ishii, 1997; 1999a; Hibiki et al.; 1998a; 1998b) that the assumption of incompressible flow could not be made for a vertical gas-liquid bubbly flow. In addition to this, the data set taken by Kashyap et al. (1994) was not accurate enough to be used for verification of the modeled sink and source terms. Therefore, the data analysis method (Ishii et al. 1997; Wu et al., 1997a; 1998b) needs to be improved and the contribution of sink and source terms to the interfacial area transport should be reexamined based on an accurate data set. Städtke et al. 
(1997) also modeled the sink and source terms in the interfacial area transport equation and tested the model with the JRC Ispra code, which was developed for two-dimensional inhomogeneous two-phase flow with the options of planar and axis symmetric conditions for quasi three-dimensional flow conditions.

In addition to the above works related to the interfacial area transport, the following studies should deserve much attention. Kalkach-Navarro et al. (1994) developed an extended two-fluid model that included the dynamics of bubble cluster formation and breakup. In their study, the transport equation to describe the bubble size distribution function was obtained by performing a balance of the loss and gain due to breakup and formation of the bubbles or bubble clusters. They found that an instability of the void wave associated with the bubble clusters triggered a bubbly/slug flow regime transition. Millies and coworkers (Millies and Mewes, 1995; Millies et al., 1996) extended the work by Kalkach-Navarro et al. (1994) to develop a first order relaxation model for the prediction of local interfacial area concentrations near the steady state. Recently, Millies and Mewes (1999) derived a simple equation for the interfacial area concentration from the population balance equation, taking into account the bubble coalescence and breakup. They applied the population balance equation to bubble columns and to vertical pipe flow in order to calculate the size distribution of the bubbles, the interfacial area concentration and the transition to plug flow. Drew and Passman (1998) discussed the modeling of the local geometry of individual elements in the dispersed component of multi-component fluids. They gave a short discussion of an empirical approach to the problem, followed by a derivation of a set of equations governing the evolution of Gaussian and mean curvature of an interface. They also proposed a simple model that accounts for the coalescence and breakup of bubbles. Recently, Morel et al. (1999) derived the local volumetric interfacial area transport equation from geometrical considerations. In the derivation, no assumption on the interface configuration was made so that the mathematical expression for the transport velocity was valid for any two-phase flow regimes.

Under the auspices of the U. S. Department of Energy, Office of Basic Energy Science, our research group at Thermal-hydraulics and Reactor' Safety Laboratory in Purdue University has also made continuous analytical and experimental efforts to develop the interfacial area transport equation. These efforts include (1) the improvement of flow measurement techniques using local sensors such as the multi-sensor conductivity probe (Kim et al., 2000) and the hot film probe (Hibiki et al., 1998a), (2) local measurements of bubbly flows in vertical round pipes to provide a rigorous data base of the interfacial area 
transport (Hibiki and Ishii, 1997; 1999a; Hibiki et al., 1998a; 1998b; 2001), and (3) the model development of sink and source terms in the one-group interfacial area transport equation (Hibiki and Ishii, 1999b; 2000a). It is recognized that these studies would give the basic understanding of the one-group interfacial area transport mechanism. On the other hand, very few studies have been performed for the development of the two-group interfacial area transport equations. The theoretical frame work of the two-group model for the interfacial area transport was proposed by Ishii and coworkers (Ishii et al., 1998; Wu et al., 1998a; Uhle et al., 1998). In their studies, although the basic mechanisms of bubble coalescence and breakup were demonstrated clearly, concrete models for the sink and source terms in the two-group transport equations were unfortunately not proposed. Recently, Hibiki and Ishii (2000b) developed two-group interfacial area transport equations and applied them to bubbly-to-slug flow successfully. This study performed (1) the formulation of the two-group interfacial area transport equations, (2) the classification of bubble interactions between spherical/distorted bubble and cap/slug bubble, (3) the categorization of the basic mechanisms of bubble coalescence and breakup, (4) the preliminary modeling of sink and source terms for the interfacial area transport equations, (5) the construction of several data sets at bubbly-to-slug flow transition by assuming the shape of a cap bubble, and (6) the validation of the two-group interfacial area transport equations by means of the data sets at the bubbly-to-slug flow transition. This study served to show the capability of the two-group interfacial area transport equations as well as to understand the basic behavior of the interfacial area transport at the transition from bubbly to slug flow.

As for the experimental study, a theoretical study carried out at Argonne National Laboratory (Kataoka et al., 1986) showed that the local interfacial area concentration could be uniquely related to the harmonic mean of the interfacial velocity. Using the theoretically supported method, the four-sensor resistivity probe method was developed to measure the interfacial area of cap/slug bubbles (Kataoka and Serizawa, 1990; Revankar and Ishii, 1993). Recently, the four-sensor probe method has been greatly improved at Purdue University (Kim et al., 1998; 1999) and will be used to provide a rigorous data base.

This final report will describe our analytical and experimental efforts on the development of the interfacial area transport equation in two-phase flow performed under the auspices of the U. S. Department of Energy, Office of Basic Energy Science. First, local measurement techniques such as multi-sensor conductivity probe and hotfilm probe methods will be explained in detail. This will include the theoretical foundation, the 
fabrication method, the signal processing method for the multi-sensor conductivity probe method, and the improved signal processing method for the hotfilm probe method. The benchmark experiment to validate these methods will also be described in detail, and the validity of these measurement methods will be demonstrated. Then, the theory of the interfacial area transport equation will be detailed. This will include the rigorous formulation of two-group interfacial area transport equations, the classification of possible bubble interaction, and the modeling of sink and source terms of interfacial area concentration. The results of the application of the interfacial area transport equation to bubbly flow and bubbly-to-slug transition flow will be explained, and the validity of the developed interfacial area transport equation will be demonstrated experimentally. 


\section{MEASUREMENT TECHNIQUES}

\subsection{Multi Sensor Probe Methodology}

In this chapter, the multi-sensor probe methodology developed in the Thermal-hydraulics and Reactor Safety Laboratory at Purdue University (Kim et al., 1999) will be explained in detail.

\subsubsection{Measurement Principle}

The measurement principle of the multi-sensor conductivity probe in obtaining local time-averaged interfacial area concentration, $a_{\mathrm{i}}$, is based on the definition given by Ishii[1], where the local time-averaged $\bar{a}_{i}^{t}$ is defined by

$\bar{a}_{i}^{t}=\frac{1}{\Delta T} \sum_{j}\left(\frac{1}{\left|v_{i} \cdot n_{i}\right|}\right)_{j}$

where $j$ denotes the $j$-th interface which passes a local point during the time interval, $\Delta T$. Here, $v_{\mathrm{i}}$ and $\boldsymbol{n}_{\mathrm{i}}$ are the bubble interfacial velocity and unit surface normal vector of the $j^{\text {th }}$ interface, respectively. Equation (2.1) implies that the local interfacial area concentration can be obtained from the bubble interfacial velocity. In view of Eq.(2.1), Kataoka et al. (1986) formulated a mathematical method to determine the local time-averaged interfacial area concentration for both double-sensor and four-sensor probes. In the application of the double-sensor probe, it was suggested that

$\bar{a}_{i}^{t}\left(x_{0}, y_{0}, z_{0}\right)=2 N_{t} \frac{1}{\left|v_{i}\right| \cos \phi}$,

with

$\frac{\bar{l}}{\left|v_{i}\right|}=\sum_{j} \frac{1}{\left|v_{i j}\right|} / \sum_{j}$ and $\frac{T}{\cos \phi}=\sum_{j} \frac{1}{\cos \phi_{j}} / \sum_{j}$,

where $N_{t}$ is the number of bubbles which pass the point $\left(x_{0}, y_{0}, z_{0}\right)$ per unit time, and $\phi_{j}=$ angle between the unit normal of the $j$-th interface and the interfacial velocity as shown in Fig.2.1. In formulating Eq.(2.2), however, it was assumed that the bubbles are spherical, and every part of the bubble has equal probability of being intersected by the probe. Also assumed was that the angle between the bubble interfacial velocity and the axial direction $z$, was random with an equal probability within some maximum angle, $\alpha_{0}$. 


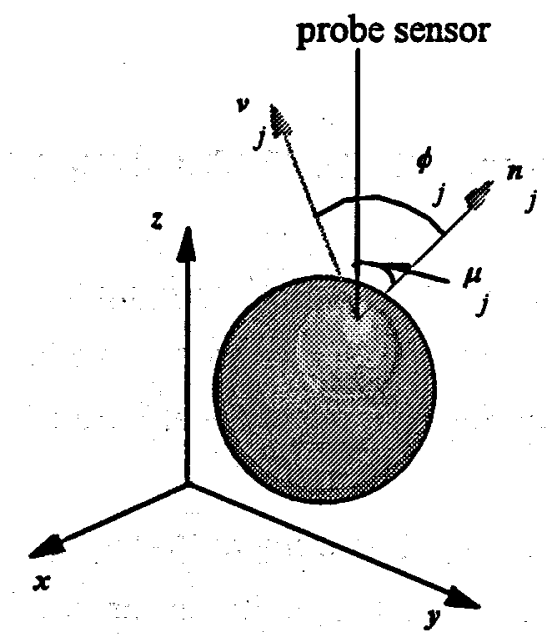

Figure 2.1. Definition of angles $\phi_{j}$ and $\mu_{j}$.

The final expression for the local time-averaged interfacial area concentration by the double-sensor probe technique was then given by (Kataoka et al., 1996)

$\bar{a}_{i}^{t}\left(x_{0}, y_{0}, z_{0}\right)=\frac{4 N_{t}\left\{\sum_{j} \frac{1}{\left|v_{s j}\right|} / \sum_{j}\right\}}{1-\cot \frac{1}{2} \alpha_{0} \ln \left(\cos \frac{1}{2} \alpha_{0}\right)-\tan \frac{1}{2} \alpha_{0} \ln \left(\sin \frac{1}{2} \alpha_{0}\right)}$,

where $\alpha_{0}$ was obtained by

$$
\frac{\sin 2 \alpha_{0}}{2 \alpha_{0}}=\frac{1-\left(\sigma_{2}^{2} /\left|v_{i z}\right|^{2}\right)}{1+3\left(\sigma_{2}^{2} /\left|\overrightarrow{v i z}^{2}\right|\right)}
$$

Here, $v_{\mathrm{szj}}, \overline{\left|\nu_{i z}\right|}$ and $\sigma_{\mathrm{z}}$ are the $z$-component of the $j$-th interfacial velocity, mean value of the $z$-component of the velocity, and the velocity fluctuations, respectively. Also, $v_{s z j}$ was defined by

$\left|v_{s j j}\right| \cos \mu_{j}=\left|v_{i j}\right| \cos \phi_{j}$,

where the angle $\mu_{j}$ was defined as the angle between the probe sensor and the normal surface vector as shown in Fig.2.1. The reciprocal harmonic mean of $\left|v_{s f}\right|$ and the square mean of fluctuation $\sigma_{z}^{2}$ were further correlated with the probability density function. For 
detailed derivation, one should refer to the study done by Kataoka et al. (1986).

In many two-phase flow systems, however, bubbles whose shapes vary significantly from spherical are frequently encountered and the application of the double-sensor probe becomes erroneous. This is where the application of a four-sensor conductivity probe becomes important. In the four-sensor conductivity probe, three pairs of double-sensor probes can be formed with one common sensor in the upstream and three independent sensors in the downstream. Therefore, three components of interfacial velocities can be obtained at a local point by measuring the time delay between the signals from three pairs of double-sensors. For example, when the directions of the three independent probes are chosen as the $x, y$, and $z$ axes, the equation for the time-averaged $a_{i}$ can be simplified as (Kataoka et al., 1986)

$\bar{a}_{i}^{t}=\frac{1}{\Delta T} \sum_{j}\left[\left(\frac{1}{v_{s 1 j}}\right)^{2}+\left(\frac{1}{v_{s 2 j}}\right)^{2}+\left(\frac{1}{v_{s 3 j}}\right)^{2}\right]^{1 / 2}$

Therefore, unlike the double-sensor probe technique, no hypothesis for the bubble shape is necessary in the mathematical formulation to calculate the local interfacial area concentration. This allows the four-sensor probe to be applied in two-phase flow regimes where bubbles are no longer spherical in shape.

Some difficulties in applying the four-sensor conductivity probe, however, have been reported by previous studies (Revankar and Ishii, 1993; Kataoka et al., 1986). The limitations were mainly caused by the size of the probe, such that the large measurement area or the spacing between the sensors caused a significant number of bubbles to miss some of the sensors. It was also reported that the deformation of the bubble interface could be significant as the bubble penetrates through the sensors. These shortcomings may lead to significant errors in estimating the local interfacial velocity. Due to such limitations, the four-sensor probe has been yet to be applicable to general experiments. Moreover, application of the four-sensor probe is limited to larger bubbles, which prevents it from being employed in general two-phase flow conditions where various sizes of bubbles exist.

\subsubsection{Development of the Miniaturized Conductivity Probe and} the Signal Processing Scheme

To minimize the reported limitations discussed above, new designs are developed for the conductivity probe. These include both modified structures of the probe and new fabrication materials, which greatly enhance the capability of the probe. The new design 
and the selection of the fabrication material are based on known shortcomings, such as deformation of bubbles, oxidation of probe sensors, and missing bubble phenomenon.

\subsubsection{Design of the Miniaturized Four-Sensor Probe}

In fabricating the probe, gold acupuncture needles with its maximum OD of 0.13-mm are employed as sensors of the probe as shown in Fig.2.2. High electrical conductivity of the gold and sharply tapered tips make the acupuncture needle ideal as sensors of the conductivity probe in reducing deformation of penetrating bubbles and minimizing the oxidation problem. For the dielectric coating of the probes, the conformal coating is used. Its low viscous ( $200 \mathrm{cps}$ at $25^{\circ} \mathrm{C}$ ), yet highly adhesive characteristics allow a very thin and uniform coating around the probe sensors with coating thickness of less than $0.05 \mathrm{~mm}$ as can be seen in Fig.2.2. It also has a high electrical resistivity of $4 \times 10^{4}$ $\mathrm{ohm} / \mathrm{cm}$, and strong water/chemical-proof characteristics, which are essential for two-phase experimental conditions. The final coating is completed using commercial copper bond epoxy so as to form a firm bond between the junction of the probe sensors and the casing. After the final coating, the conductive ink is painted at the junctions to bond and to ensure electrical connections. A gauge $11(0.318-\mathrm{cm}$ OD) stainless steel tube is used for the probe casing and it is reduced to a gauge 14 stainless steel tube to hold the sensor assembly. The casing is bent exactly at $90^{\circ}$ without curvature.

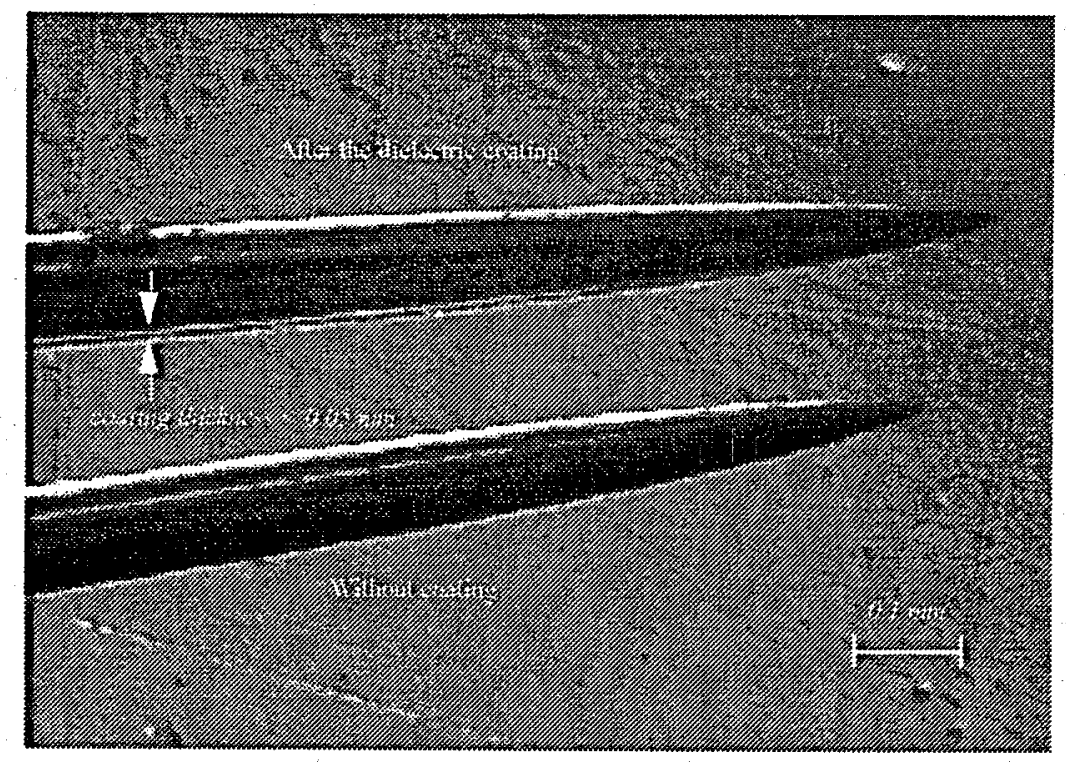

Figure 2.2. Photographic image of the probe sensors with/without dielectric coating. 
Both the coaventional and new designs of the probes are shown in Fig.2.3. The significant reduction in the cross-sectional measurement area of the newly designed probe and its sharply tapered tips of the sensors can effectively minimize both the number of missing bubbles and the deformation of passing bubble interfaces. In fact, in the present experiments using the newly designed four-sensor conductivity probe, very few missing bubbles are found.

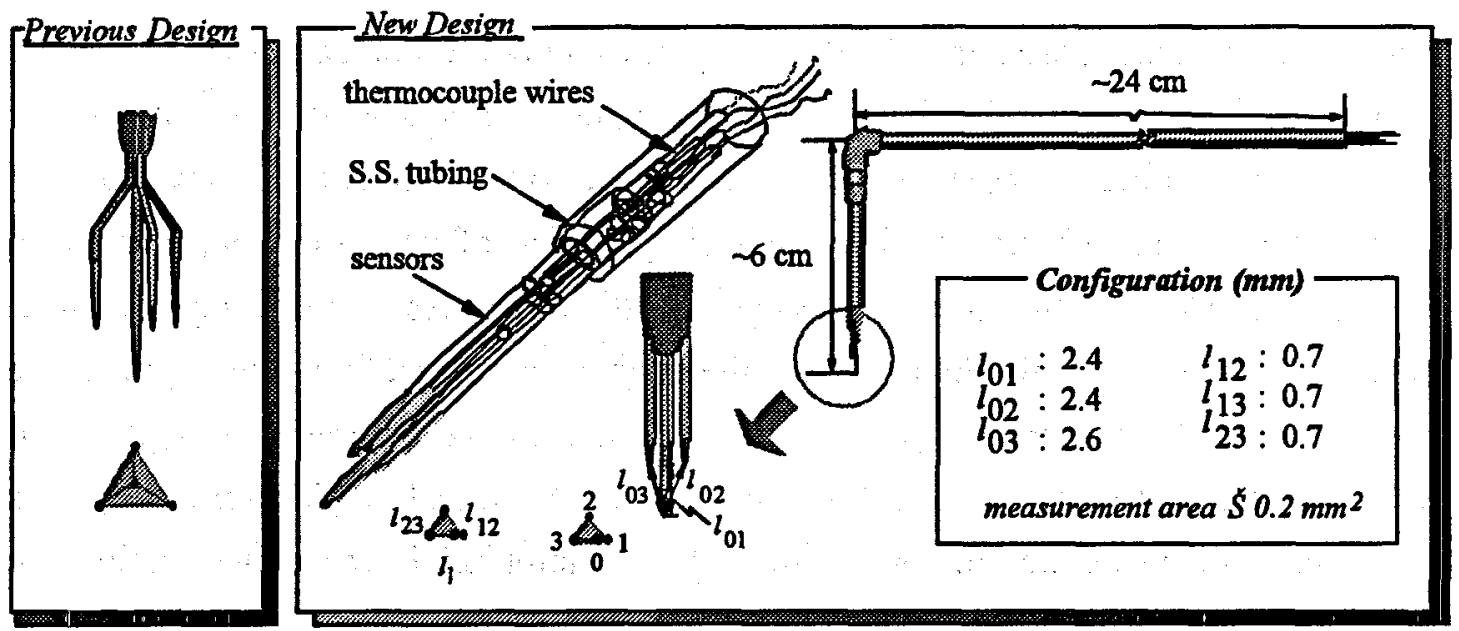

Figure 2.3. Schematic diagrams of the conventional and new four-sensor conductivity probes.

Another significant feature of the newly design probe is that it accommodates a built-in double-sensor probe in the four-sensor configuration. Therefore, the newly designed four-sensor probe can be applied in a wide range of two-phase flow regimes spanning over bubbly, cap, slug, and churn-turbulent flows. This is a significant improvement in view of the interfacial area transport, because it allows one to observe how the local two-phase flow parameters develop along the flow field as the bubble size changes. The measurement area of the four-sensor probe used in the experiments is less than $0.2 \mathrm{~mm}^{2}$ and the distance between the two tips of the double-sensor probe (sensors 0 and 1 in Fig.2.3) is $2.4 \mathrm{~mm}$. Here, the tip distance in the double-sensor part of the probe is based on the study by Wu and Ishii (1999). Hence, with the present probe configuration, the probe's range of measurable bubble diameter is from $\sim 1 \mathrm{~mm}$ to Taylor bubbles.

In acquiring the signals from the sensors, a simple DC circuit is employed for 
simplicity of the device and for faster rise/fall time in the signals. It has been verified through experiments that the newly designed probe would last more than 1 year provided that the sensors are dried in the air after the experiments. The sampling frequency is varied between 6 to $10 \mathrm{kHz}$ depending on the flow conditions, and an average of more than 2,000 small bubble and 200 large bubble signals are acquired in slug flow conditions.

\subsubsection{Signal Processing Scheme}

The signal processing scheme developed for the newly designed conductivity probe is structured in two main parts; namely, the first part for conditioning acquired signals and the second part for processing the signals to obtain the desired two-phase flow parameters. The signal conditioning process includes filtration, normalization, and conversion of signals into step signals, whereas the data processing consists of categorization, calculation, and correction processes. In processing the signals, the principles of obtaining interfacial area concentration with signals obtained from the double-sensor and four-sensor probes are different due to the fundamental difference in bubble shapes, measurement principles, and the geometry of the probes. In the following sections, the signal conditioning processes and the calculation principles of local time-averaged interfacial area concentration are discussed.

\section{(A) Signal Conditioning}

To obtain accurate local two-phase flow parameters, it is critical to identify the signals from the bubbles accurately. In order to accomplish this successfully, the signal processing scheme is constructed in several steps. They include; moving median filtering process of the raw signals, normalization of the filtered signals, filtering noises through noise threshold, and generation of step signals.

It is likely that the signals obtained from the probe sensors contain some characteristic high frequency noises from other electronic devices. In such a case, the high frequency noises should be effectively removed to prevent significant error in estimating the interfacial area concentration. In order to filter these high frequency noises, the moving median filter scheme is employed. The algorithm of the moving median filter can be explained as follows; First, the initial data point of interest is selected. A consecutive odd number of data points ( 3 or bigger) surrounding the point of interest are then selected as a group. In the given group of data points, the median value is found. This median value is then registered as the data point of interest in the filtered set of data. The next group is selected by advancing one data point from the previous data point of interest and grouping 
odd numbers of data points surrounding the new data of interest. This process is repeated until the final data point is reached. In the present experiments, the five-point moving median filter is employed. However, any odd number of data points equal or bigger than 3 can be chosen as a group for this filtering process.

Due to the finite rise/fall time of the signal and the different experimental conditions, neither the absolute value of the base voltage, nor the voltage drop is fixed. Therefore, all the signals obtained by the probe sensor should be normalized. This normalization can be calculated using

$$
V_{\text {nom }, i}=\frac{V_{i}-V_{\text {mix }}}{V_{\text {max. }}-V_{\text {min }}}
$$

where $V_{\text {norm, }}$ is the normalized voltage of the $i$-th signal, $V_{\mathrm{i}}$ is the $i$-th signal, $V_{\max }$ is the maximum voltage, and $V_{\min }$ is the minimum voltage. Here, the minimum voltage is determined by the average voltage signal in the liquid phase. A simple way to determine the minimum voltage is to divide the total voltage range into four quarters, and set the most probable voltage in the lowest quarter as $V_{\min }$.

After the normalization, the remaining noises are removed by setting a threshold level. In principle, this level can be determined by finding the standard deviation of the fluctuations due to noise. In practice, however, the threshold level determined by an experimental observation can be acceptable if the fluctuations are not severe. In the present experiment, the threshold level of $\pm 0.05 \mathrm{~V}$ determined by experimental observation was an adequate level to remove noises. Therefore, any voltage fluctuations within \pm 0.05 $\mathrm{V}$ are considered to be noise and are removed from the raw signals.

If the signals rise or fall instantaneously without any time delay when the bubbles pass through the probe sensors, the registering signals would be in step function form and the interfaces can be identified definitely. However, due to the finite rise/fall time in the signals, ambiguities in identifying the bubble interfaces can arise. Therefore, it is necessary that the raw signals obtained by the probe sensors be converted into step signals. In the present signal processing scheme, the criterion for converting the normalized signals into the square signals is constructed by determining the initiation of the bubble interface. When the acquired signal keeps rising (falling) more than 5 data points continuously above (below) the noise level, it is considered to be due to the bubble interface. After the bubble interface is detected, the signal is converted into a step function at the initial points. An example of signals converted from the normalized signals into the step signals is shown in Fig.2.4. 


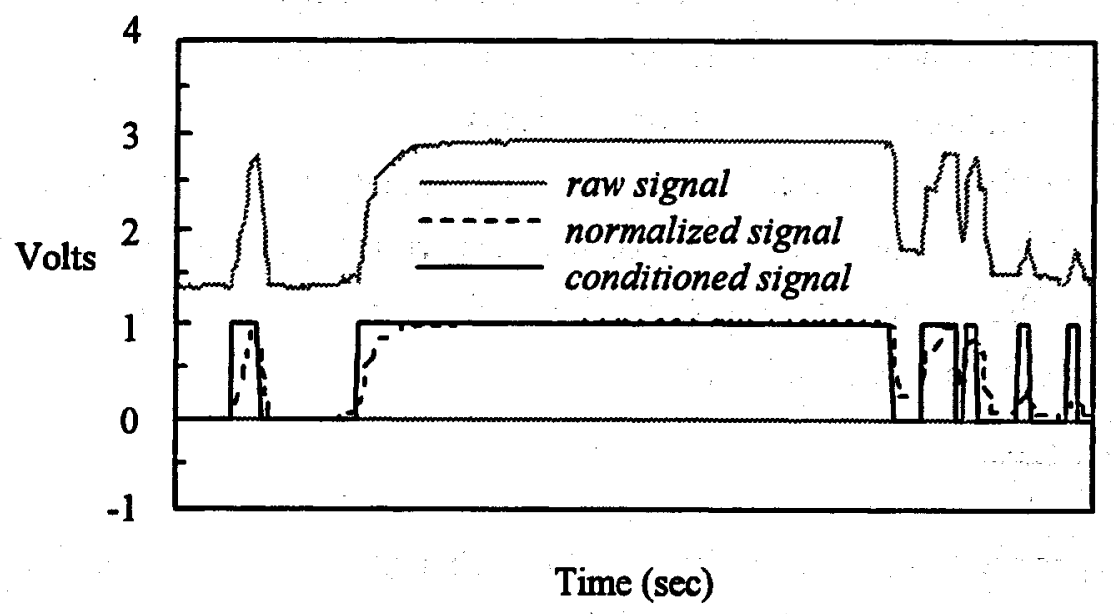

Figure 2.4. The signals before and after the signal conditioning processes.

\section{(B) Categorization of Bubble Signals}

After the completion of the signal conditioning process, the signals are separated into signals of spherical, distorted, cap, and Taylor bubbles depending on the bubble chord length information. This categorization process is possible due to the improved design of the new four-sensor probe, which accommodates both the double-sensor and four-sensor probes. This categorization process is important in view of interfacial transport, because it allows one to study the transport phenomena of different types of bubbles independently.

In the present experiments, spherical and distorted bubbles are categorized as group I, and the cap and Taylor bubbles are categorized as group II. In identifying the bubble types, the maximum distorted bubble limit and the spherical bubble limit given by Ishii (1977), and Ishii and Zuber (1979) are used as criteria, such that

$D_{d s}=4 \sqrt{\frac{2 \sigma}{g \Delta \rho}} N_{\mu_{f}}^{1 / 3} ;$ spherical bubble limit

and

$D_{d \max }=4 \sqrt{\frac{\sigma}{g \Delta \rho}} ;$ maximum distorted bubble limit

where 
$N_{\mu f}=\frac{\mu_{f}}{\left(\rho_{f} \sigma \sqrt{\frac{\sigma}{g \Delta \rho}}\right)^{1 / 2}}$.

Therefore, the bubbles whose chord lengths are smaller than $D_{\mathrm{ds}}$ are categorized as spherical bubbles, and those bigger than $D_{\mathrm{ds}}$ but smaller than $D_{\mathrm{dmax}}$ are categorized as distorted bubbles. In the present experiment, the Taylor bubble is defined as the bubble whose diameter is the same as the tube diameter. Hence, when the chord length of the bubble at the center of the tube is the same or bigger than the tube radius, it is categorized as a Taylor bubble. The cap bubbles are then categorized as those whose chord lengths are bigger than $D_{\text {dmax }}$ and smaller than the Taylor bubble chord length.

In obtaining the void fraction and the bubble chord length, the common sensor (sensor 0 in Fig.2.3) is used. For the interfacial area concentration, the signals from the double-sensor probe (sensors 0 and 1 in Fig.2.3) are used for the spherical bubbles, whereas the signals from three pairs of double-sensor (sensors $0 \& 1,0 \& 2$, and $0 \& 3$ in Fig.2.3) are used otherwise.

\section{(C) Calculation of the Time-Averaged Interfacial Area Concentration}

The measurement principle of the multi-sensor conductivity probe in obtaining local time-averaged interfacial area concentration, $a_{i}$, is based on the definition given by Ishii (1975) as shown in Eq.(2.1). Based on this definition, Kataoka et al. (1986) formulated a mathematical method to determine the local time-averaged $a_{\mathrm{i}}$ for both double-sensor probe and four-sensor probes by measuring the local interfacial velocity as shown in Eqs.(2.4) and (2.7), respectively. Since then, the equations have been improved (Kataoka et al., 1994; Wu and Ishii, 1999) to account for the errors associated with the measurement errors due to the limitations of the probe(Revankar and Ishii, 1993; Kataoka et al., 1994; Wu and Ishii, 1999). Such efforts have been made particularly on improving the double-sensor conductivity probe measurement. This is due to the fact that the measurement technique employing the double-sensor probe requires assumptions such as; The bubbles are spherical in shape; Every part of the bubble has an equal probability of being intersected by the probe; The angle between the bubble interfacial velocity and the axial direction, $z$, is random with an equal probability within some maximum angle, $\alpha_{0}$; and There is no fluctuation in the path of the penetrating bubble, and it passes through both sensors of the probe.

For the measurement employing the four-sensor probe, on the other hand, other phenomena should be carefully examined due to the characteristic shape of the large 
bubbles. When the bubble size is large compared to the separation distances of the sensors ( $l_{1 \mathrm{i}}$ where $\mathrm{i}=1,2$ and 3 in Figure 4 ) and when the bubble interface is not significantly distorted, the bubble penetrates all four sensors. Such an event registers four consecutive signals from front and rear interfaces of passing bubbles, and the three components of the local interfacial velocity from front and rear interfaces can be calculated by the time delay information obtained from the signals. However, the interfaces of large bubbles are susceptible to significant distortions. Furthermore, in slug flow conditions, one of the probe sensors may miss the interface near the flow duct wall due to the characteristic liquid film formed near the wall.

In view of these, three kinds of bubble signals can be identified as signals that cannot be used in obtaining the local instantaneous interfacial velocity. The first kind occurs when a small spherical bubble misses one sensor of a double-sensor probe. In this case, only a single signal is registered, as illustrated in Fig.2.5(a). The term 'missed' or 'missing' bubble signal refers to this case. The second kind is referred to as a 'non-effective' bubble signal. When the interfaces of the bubbles are highly distorted, the signals due to the rear interfaces of the bubbles obtained by the common sensor may not precede the ones obtained by other rear sensors of the probe. When such signals are acquired, it is not possible to estimate the interfacial velocity, and those 'non-effective' signals cannot be used in calculating the local $a_{\mathrm{i}}$. An example of such signals is illustrated in Fig.2.5(b). The third kind occurs due to the characteristic shape of Taylor bubbles. In slug flow conditions, a thin layer of liquid film exists between the Taylor bubble and the flow duct wall, by which a very steep interface is formed. When such a steep interface is encountered, the interface and the probe sensors become parallel to each other, and one of the probe sensors may miss the interface. An example of missing signals due to the steep Taylor bubble interface is shown in Fig.2.5(c). This steep Taylor bubble interface is of great importance, because the major contribution in the interfacial area concentration of the Taylor bubbles is attributed to this interface. Therefore, proper estimation of such missing signals should be made. This missing phenomenon near the flow duct wall due to the steep interface of Taylor bubbles was also reported by Ishii and Revankar (1993).

Recently, Wu and Ishii (1999) suggested a correction method accounting for the missed interfaces of spherical bubbles in the application of the double-sensor conductivity probe. In this study, they considered the effects of the lateral movement of the bubbles and the probe tip spacing $\left(l_{01}\right.$ in Fig.2.3). They divided the measured bubbles in two categories, one for bubbles whose interface is moving normal to the probe and passing through both 
sensors, and another for those missing one of the sensors of the probe. In their correction scheme, the mean value of the experimentally measured bubble interfacial velocity was rigorously related to the actual interfacial velocity of the bubbles by defining theoretical calibration factors. These calibration factors were employed to account for the bubbles whose interfaces move normal to the probe, and those missing one of the sensors. By determining the calibration factors, they modified the formula given by Kataoka et al. (1986) as

$\bar{a}_{i}^{t}=f_{\text {total }}\left(\frac{2 N_{b}}{\Delta s \Delta T}\right)\left(\frac{\sum_{j}\left(\Delta t_{j}\right)}{N_{b}-N_{\text {miss }}}\right)$, for $\Delta s=0.36 D_{\mathrm{b}} \sim 0.86 D_{\mathrm{b}}$

with

$f_{\text {total }}=2+\left(\frac{v_{b}^{\prime}}{v_{b}}\right)^{225}$, for $\Delta s=0.36 D_{\mathrm{b}} \sim 0.86 D_{\mathrm{b}}$

where $N_{b}$ is the number of total bubbles obtained, $v_{b}^{\prime}$ is the fluctuation of bubble velocity, $\bar{v}_{b}$ is the average bubble velocity obtained by effective signals, and $\Delta t_{j}, \Delta T, \Delta s$ are the time delay obtained by effective signals for the $j$-th bubble interface, total sampling time at a local point, and distance between two tips of the sensors, respectively. Equation (2.12) was found to be valid as long as the output signals from the probe were valid for bubble identification and the sample size was sufficiently large. For bubble sizes varying from 0.6 to 1.4 times the mean bubble size, it was found that the interfacial area concentration calculated by Eq.(2.12) would result in a statistical error of $\pm 7 \%$ for a sample size of $\sim 1,000$ bubbles (Wu and Ishii, 1999).

For bubbles whose shapes are not spherical, such as distorted, cap, or Taylor bubbles, the local time-averaged interfacial area concentration is obtained by the signals acquired from four sensors. Due to the irregular interfaces of larger bubbles, the contribution to $a_{\mathrm{i}}$ from the front and rear interfaces can be significantly different. Therefore, each interface should be considered separately. Hence, unlike the double-sensor method, each signal pair from the front and rear interface is considered independently in the calculation of interfacial area concentration. In the process of estimating the local interfacial velocity with the defective signals from distorted and cap bubbles, the corrections are made in two steps for both the non-effective signals and missing signals. First, when the non-effective rear signals are encountered, the corrections 
are made according to the average $a_{\mathrm{i}}$ acquired from effective rear signals as well as the total number of non-missing bubbles. Next, the missing bubble signals are corrected by the average contribution from both the effective and the total number of bubbles. This two-step correction method yields the equation for the total time-averaged $a_{i}$ at a local point as

$\bar{a}_{i, \text { tot }}=\bar{a}_{i f, \text { eff }}\left(\frac{N_{\text {tot. }}}{N_{f, \text { eff }}}\right)+\bar{a}_{i r, \text { eff }}\left(\frac{N_{\text {tot }}}{N_{r, \text { eff }}}\right):$ for distorted and cap bubbles

where $\bar{a}_{i, \text { eff }}$ is the average $a_{i}$ calculated with effective bubble signals, $N_{\text {eff }}$ is the number of effective bubble signals, $N_{\text {tot. }}$ is the total number of bubbles counted by the common sensor, and $f \& r$ denote front and rare bubble interfaces, respectively.

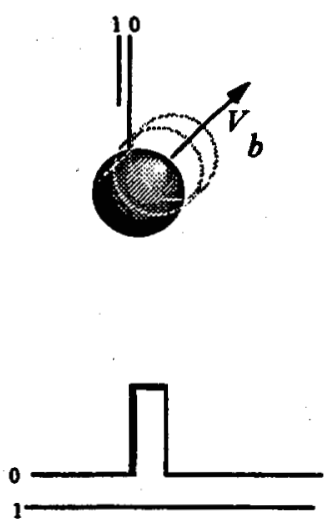

Missing Signal (Spherical Bubbles)

(a)

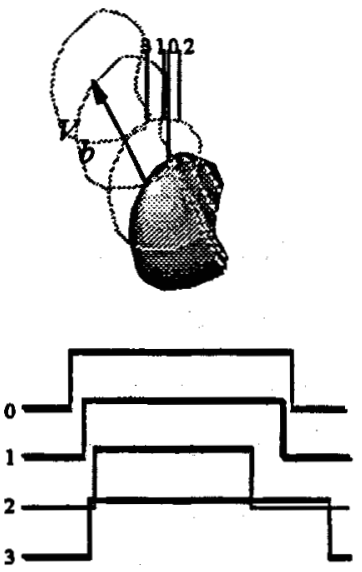

Non-effective Signal (Non-Spherical Bubbles)

(b)
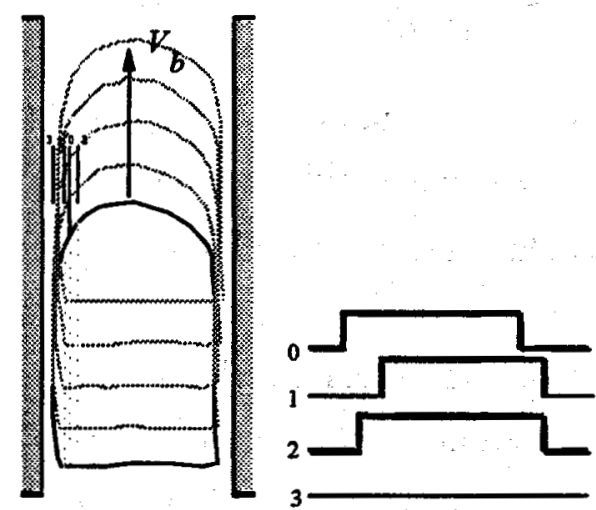

Missing Signal Due to Steep Interface (Slug Bubbles)

(c)

Figure 2.5. Examples of defective signals.

In order to account for the missing signals due to the steep interface of Taylor bubbles near the flow duct wall, the correction method employed by Ishii and Revankar(1993) can be applied. In their study, the interfacial area concentration was treated as the ratio of the surface area of the bubble interface to the volume formed by the measurement area of the four-sensor probe. The equation for the calculation of $a_{i}$ of the 
missing Taylor bubble interfaces was then given by

$\bar{a}_{i, \text { miss }}=N_{\text {miss }} \cdot \frac{t_{b}}{\Delta T} \cdot \frac{\bar{l}_{s}}{A_{s}}$

where $N_{\text {miss }}$ is the number of missing (steep) Taylor bubble interface, $t_{\mathrm{b}}$ is the residence time of the missing bubble signals, $\Delta T$ is the total sampling time, $\bar{l}_{s}$ is the average distance between three independent sensors (i.e., $l_{12}, l_{13}$, and $l_{23}$ in Fig.2.3), and $A_{\mathrm{s}}$ is the measurement area of the probe. Therefore, the total time-averaged $a_{\mathrm{i}}$ for the Taylor bubbles is obtained by

$\bar{a}_{i, \text { slug,tot }}=\bar{a}_{i, \text { off }}\left(\frac{N_{\text {tot }}}{N_{f, \text { eff }}}\right)+\bar{a}_{i r, \text { eff }}\left(\frac{N_{\text {tot }}}{N_{r, \text { eff }}}\right)+\bar{a}_{i, \text { miss }}:$ for Taylor bubbles

In Eq.(2.16), it should be noted that $N_{\text {tot. }}$ is the sum of the effective and non-effective interfaces without including the missing Taylor bubble interfaces.

In summary, followings are the equations used in calculating the local time-averaged $a_{\mathrm{i}}$ in the present experimental study: Equation (2.12) is used in the calculation of the total $a_{\mathrm{i}}$ for the spherical bubbles using the signals obtained by the double-sensor probe. The equation given by Kataoka et al. (1986) for the four-sensor conductivity probe is used in calculating $a_{i}$ for distorted, cap, and Taylor bubbles with effective signals. Equations (2.14) and (2.16) are used in the calculation of the total $a_{\mathrm{i}}$ for distorted \& cap bubbles, and the total $a_{\mathrm{i}}$ for the Taylor bubbles, respectively. The local time-averaged two-phase parameters obtained after the completion of the signal processing are: number of bubbles, interfacial velocity, void fraction, interfacial area concentration, and Sauter mean diameter of each type of bubbles. The two-group parameters are obtained by summing the corresponding parameters, namely; group I by summing the acquired parameters from the spherical and distorted bubbles, and group II by summing those from the cap and Taylor bubbles.

\subsubsection{Benchmark Experiment}

A schematic diagram of the vertical co-current two-phase flow experimental loop is shown in Fig.2.6. The test section is made of a Lucite pipe of $5.08 \mathrm{~cm} \mathrm{ID}$ with $375 \mathrm{~cm}$ in height. The de-mineralized water and air are supplied by a centrifugal pump and an air-compressor, respectively. Bubbles are generated through stainless steel hypodermic tubes of ID $0.12 \mathrm{~mm}$, which are arranged in a $20 \times 20$ square matrix. After the two-phase mixing chamber, a Tee is employed to create larger bubbles. Bubbles escaping from the air 
chamber in the Tee assembly are then introduced into the test section through a horizontal tube followed by two $45^{\circ}$ elbows. The liquid and gas flow rates are controlled by rotor meters. The probe is mounted on a support, which is designed such that the probe can be traversed by a micrometer. To capture images of bubbles, a SONY CCD VX-3, Hi-8 video camera is used at a frame rate of 30 frames $/ \mathrm{sec}$.

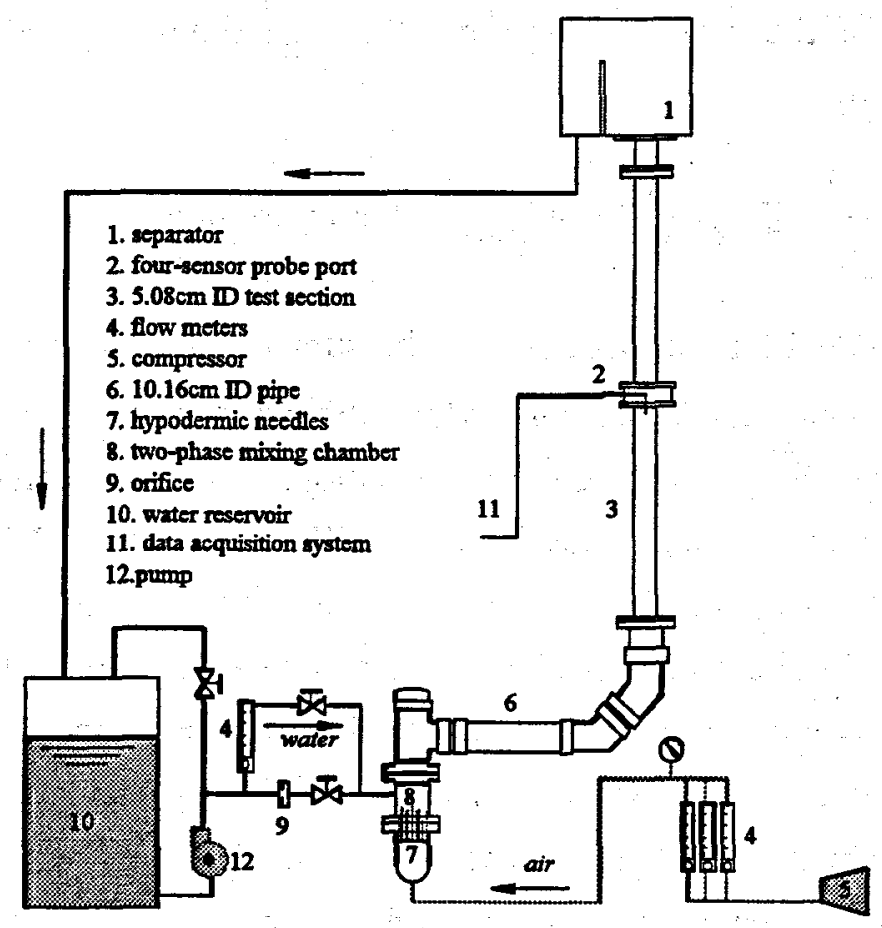

Figure 2.6. Schematic diagram of the experimental loop designed for the benchmark experiment of the newly-designed miniaturized four-sensor conductivity probe.

The gas flow rates are varied by $\left\langle j_{B}\right\rangle=0.052,0.179$, and $0.432 \mathrm{~m} / \mathrm{s}$ while the liquid flow rate is fixed at $\left\langle j_{\mathrm{p}}\right\rangle=0.321-\mathrm{m} / \mathrm{s}$. The flow conditions are chosen such that the Taylor bubbles start to form at the lowest gas flow condition at $z D=32$. A simple $D C$ circuit is employed to obtain the signals from the probe. The signal from the probe is imported to the personal computer by a data acquisition system. The imported signal is then processed by a personal computer. The sampling frequency is varied between 6 to $8 \mathrm{kHz}$ depending on the flow conditions. More than 2,000 of group I bubbles and more than 200 group II bubbles are acquired at a local measurement point for the given sampling time. The probe 
is traversed at a fraction of $1.27 \mathrm{~mm}$ from the center to the wall of the test tube. The local time-averaged interfacial area concentrations for spherical bubbles are acquired from the signals obtained by the built-in double-sensor unit, and that of other types of bubbles is acquired by the signals from four sensors of the probe.

The characteristic experimental results obtained by the four-sensor conductivity probe are shown in Fig.2.7(a) through (c). The data shown in these figures are obtained at $z / D=32$ with $\left\langle j_{\mathrm{g}}\right\rangle=0.432-\mathrm{m} / \mathrm{s}$ and $\langle j \mathrm{p}\rangle=0.321-\mathrm{m} / \mathrm{s}$. At this flow condition, the average chord length of the Taylor bubbles measured at the center of the flow duct by the common sensor is $17.2 \mathrm{~cm}$. In Fig. 2.7(a), the profiles of the local time-averaged void fraction along the radial direction of the tube are plotted. Here, the void fractions are categorized into two groups, where group I includes the spherical and distorted bubbles and group II includes cap and Taylor bubbles. Also plotted in this figure is the total void fraction, which is the sum of the void fraction of two groups. The void fraction of the group II bubbles peak at the center of the tube, while that of the group I bubbles remains nearly uniform across the flow duct. Under this flow condition, the contribution to the void fraction from group I bubbles is about $20 \%$ of the total void fraction, and the total void fraction is determined mainly by the group II bubbles. The profiles of the local time-averaged interfacial area concentration are shown in Fig.2.7(b). In the interfacial area concentration profiles of the group II bubbles, a local peak near the pipe wall is observed. Unlike the void fraction, the major contribution to total interfacial area concentration is due to the group I bubbles. To highlight the contribution of the Taylor bubbles, the local time-averaged void fraction and

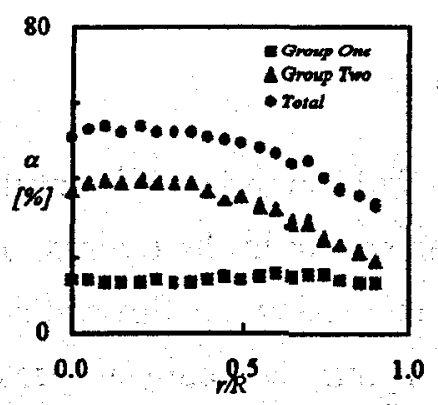

(a)

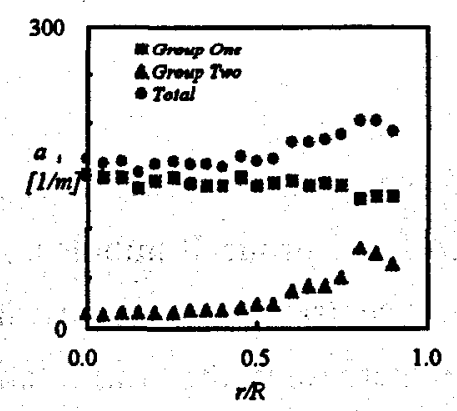

(b)

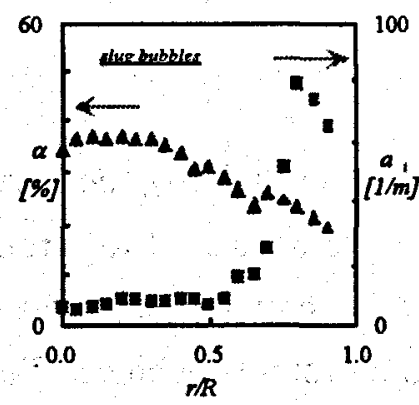

(o)

Figure 2.7. The characteristic experimental results obtained by the four-sensor conductivity probe at $z / D=32$ with $\left\langle j_{\bar{B}}\right\rangle=0.432 \mathrm{~m} / \mathrm{s}$ and $\left\langle j_{\mathrm{p}}\right\rangle=0.321 \mathrm{~m} / \mathrm{s}$. The time-averaged local (a) void fraction and (b) interfacial area concentration of group I and group II bubbles, and (c) for Taylor bubbles. 
the interfacial area concentration of the Taylor bubbles are plotted in Fig.2.7(c). The center peak in the void profile and the sharp wall peak in the interfacial area concentration profile clearly demonstrate the characteristic signature of the Taylor bubbles. Comparing Fig.2.7(c) with (b), it can be seen that the void fraction of the group II bubbles is determined mainly by the Taylor bubble.

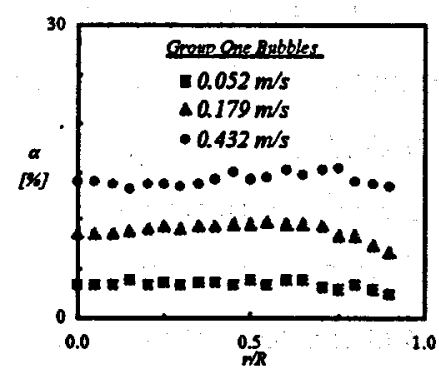

(8)

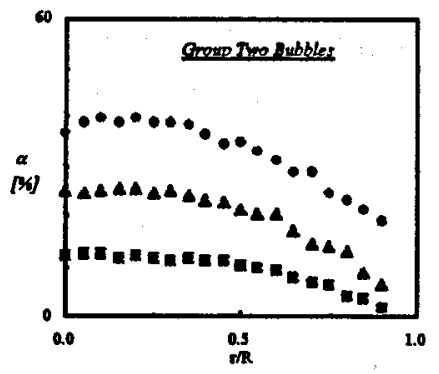

(c)

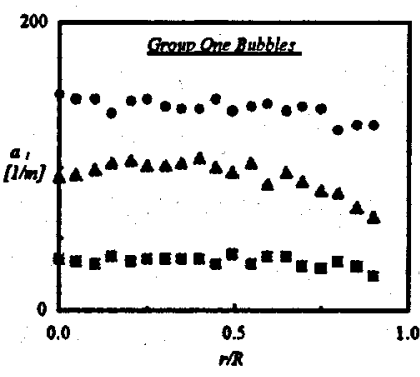

(b)

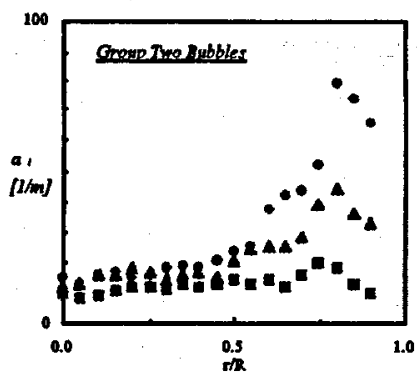

(d)

Figure 2.8. Local time-averaged void fraction and interfacial area concentration in three different flow conditions for (a) \& (b) for group I and (c) \& (d) group II bubbles; $\left\langle j_{\mathrm{p}}>=0.321 \mathrm{~m} / \mathrm{s} \&\left\langle j_{\mathrm{g}}>\right.\right.$ varied.

In Fig.2.8(a) through (d), the local two-phase parameters at $z / D=32$ in three different flow conditions are compared for group I and group II bubbles. As shown in the figures, both void fraction and interfacial area concentration increase with increasing gas flow rates for group I and group II bubbles. The increase in the void fraction and the local wall peak in the interfacial area concentration profile of group II bubbles indicate that the length of the Taylor bubble becomes longer, and the contribution from the side interface of the Taylor bubbles in local $a_{\mathrm{i}}$ becomes more significant with increasing gas flow rates. Moreover, the local $a_{\mathrm{i}}$ of the group II bubbles remains unchanged between the tube center and $r / R \bumpeq 0.5$.

This implies that the shapes of the nose of Taylor bubbles do not change 


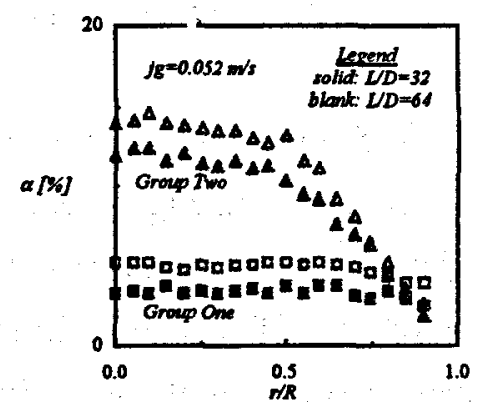

(a)

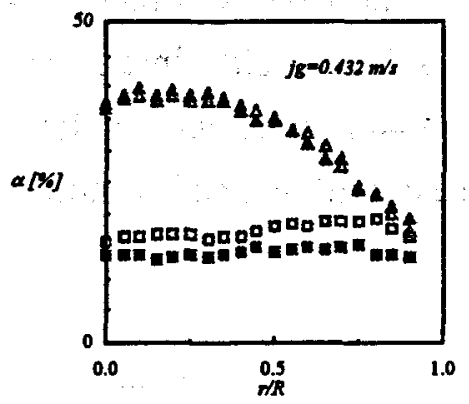

(c)

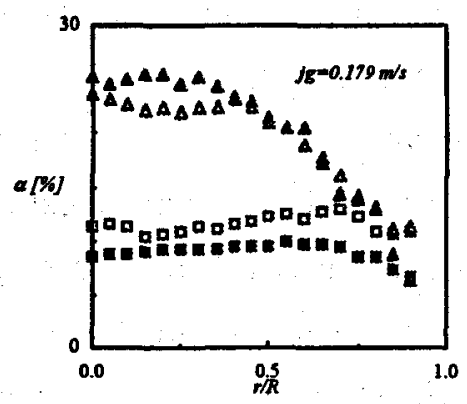

(b)

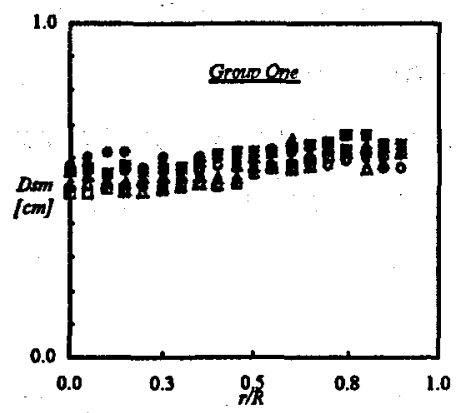

(d)

Figure 2.9. Development of void fraction and Sauter mean diameter along the axial direction of the flow duct. Solid data points measured at $L D=32$ and blank data points measured at $z / D=64$. $\left\langle j_{\mathrm{f}}\right\rangle=0.321 \mathrm{~m} / \mathrm{s}$ and (a) $\left\langle j_{\mathrm{g}}\right\rangle=0.052 \mathrm{~m} / \mathrm{s}$, (b) $\left\langle j_{\mathrm{g}}\right\rangle=0.179 \mathrm{~m} / \mathrm{s}$, (c) $\left\langle j_{\mathrm{B}}>=0.432 \mathrm{~m} / \mathrm{s}\right.$, and (d) Sauter mean diameters of group I bubbles.

significantly regardless of the bubble chord length.

In view of axial development of the two-phase parameters, the local void fraction and the interfacial area concentration measured at $z D=32$ and 64 are plotted in Figures 2.9 and 2.10, respectively. In Figs.2.9(a) through (c), the local time-averaged void fraction of group I and group II bubbles measured at $z / D=32$ and 64 are compared for three different flow conditions. In Fig.2.9(d), profiles of the time-averaged local Sauter mean diameter of group I bubbles for all cases are plotted. Here, the Sauter mean diameter is calculated by $D_{s m}=\frac{6 \alpha}{a_{i}}$

As shown in Fig.2.9(a) through (c), the void fraction of group I bubbles increases along the axial direction for all flow conditions. The Sauter mean diameters of group I bubbles, however, remain nearly uniform regardless of both flow conditions or axial locations. This implies that both the interfacial area concentration and void fraction of the group I bubbles 
increase. Hence, the number of group I bubbles increases with increasing gas flow rates and along the downstream of the flow duct.

In Fig.2.10, the void fraction and interfacial area concentration for the Taylor bubbles are plotted for two different axial locations. As shown in Fig.2.10(a), the void fraction of Taylor bubbles increases with increasing axial location, whereas in (b) and (c) it remains nearly the same. This is due to the coalescence of Taylor bubbles in the downstream, which results in the decrease in the number of Taylor bubbles and increase in the Taylor bubble length. Also, the local peak in the $a_{\mathrm{i}}$ near the wall increases along the axial direction in all flow conditions, which reflects longer side interfaces of Taylor bubbles. The $a_{\mathrm{i}}$ near the center of the tube, however, remains quite uniform for all the flow conditions suggesting that the contour of the slug nose doesn't depend significantly on the length of the Taylor bubble.

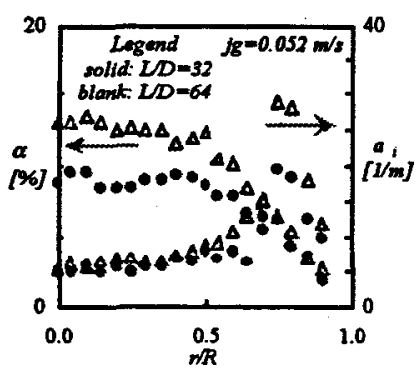

(a)

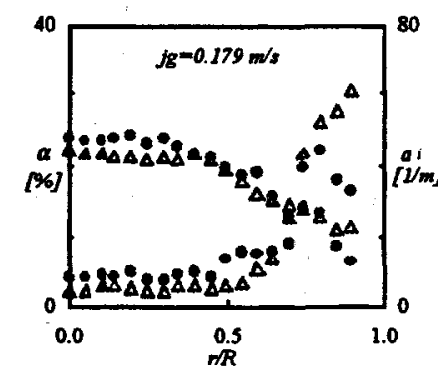

(b)

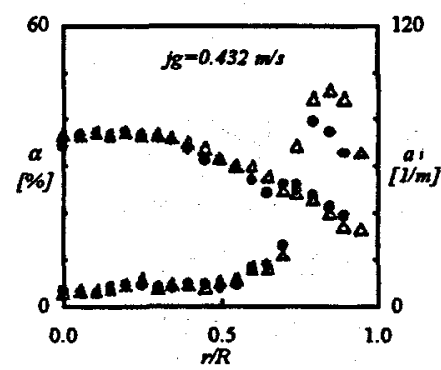

(c)

Figure 2.10. Development of two-phase parameters for Taylor bubbles in three different flow conditions. Solid data points at $z / D=32$ and blank data points at $z / D=64$.

\subsubsection{Benchmark of the New Four-Sensor Conductivity Probe}

In order to benchmark the newly developed conductivity probes and its signal processing scheme, an image analysis method is employed. In benchmarking the double sensor conductivity probe, the images captured in a bubbly flow is analyzed, whereas the Taylor bubble images are used in benchmarking the four-sensor conductivity probe.

\subsubsection{Benchmark of the Double-Sensor Conductivity Probe}

The benchmark experiments for the double-sensor probe is performed in an acrylic vertical rectangular flow duct in air-water two-phase mixture (Wu et al., 1997b). The rectangular flow duct is ideal in view of reducing image distortion. A rectangular 
window of $100 \times 62-\mathrm{mm}$ in size is prepared at the same location where the measurements are made by the double-sensor conductivity probe. The images of the bubbly flow are captured by a SONY CCD VX-3, Hi-8 video camera at a frame rate of 30 frames $/ \mathrm{sec}$. The void fraction of the two-phase mixture is controlled, so that it is below $5 \%$ in order to prevent the bubble images from overlapping. A computer code developed by $Z$ hang and Ishii (1995) is used to process the captured images to obtain the location and the diameter of each bubble in a given frame. An example of the photographic images used in image analysis and the coordinate system employed in the image analysis is shown in Fig.2.11. Here the image is captured at the flow condition of $\left\langle j_{g}\right\rangle=0.023-\mathrm{m} / \mathrm{s}$ and $\left\langle j_{p}\right\rangle=0.315-\mathrm{m} / \mathrm{s}$. The $y-z$ plane, or (flow duct depth) $\times$ (window height), area-averaged interfacial area concentration at any $x$ position is then calculated by

$\left\langle a_{i}(x)\right\rangle_{y z}=\frac{\pi \sum_{j}\left(D_{b}(x)\right)_{j}}{A_{y z}}$

where $D_{\mathrm{b}}(x)$ is the bubble diameter at location $x$, and $A_{\mathrm{yz}}$ is the product of image height and flow duct depth. In order for the data obtained by the double-sensor conductivity probe to be compared effectively, the local time averaged interfacial area concentration acquired

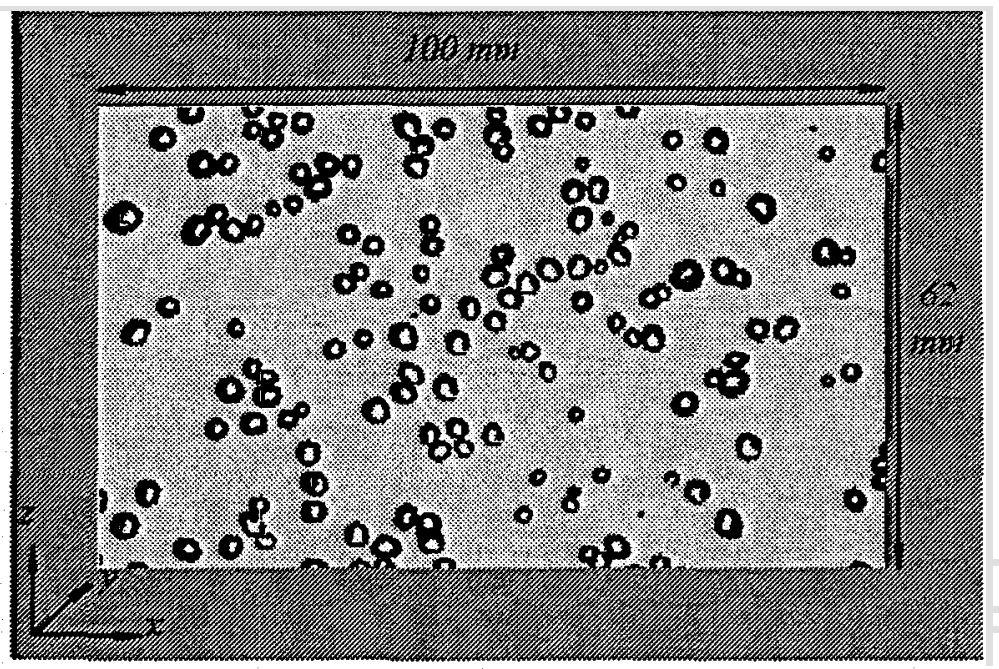

Figure 2.11. Photographic image captured for image analysis in benchmarking the double-sensor conductivity probe and its coordinate system. $\left\langle j_{B}>=0.023 \mathrm{~m} / \mathrm{s}\right.$ and $\left\langle j_{p}>=0.315 \mathrm{~m} / \mathrm{s}\right.$. 
along the $x$-direction should be line-averaged along the $y$-direction. Therefore, in obtaining the experimental values, the probe is traversed in half-width of the flow duct gap from the center to the wall by a fraction of 1-mm assuming that the flow is symmetric along the $y$-direction. The equivalent values of the interfacial area concentration measured by the probe to the values from image analysis method is then given by

$\left\langle a_{i}(x)\right\rangle_{y, \text { probe }}=\frac{I}{L_{y}} \int_{0}^{L_{y}} \bar{a}_{i}^{t} d y$,

where $L_{\mathrm{y}}$ is the total length of the flow duct gap width.

The typical result from a number of experiments is shown in Fig.2.12. The relative percent difference between the two-methods is within $\pm 10 \%$. Considering the limitation of the image method near the edge of the viewing window (as shown in Fig.2.11), the agreement is acceptable.

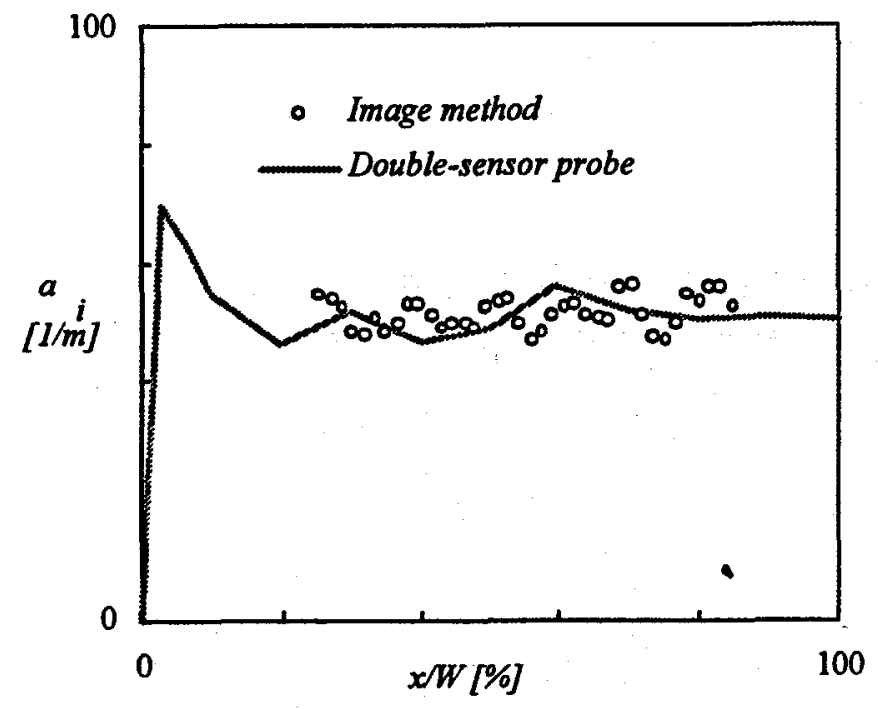

Figure 2.12. Typical results obtained from the comparison between the interfacial area concentration measured by the double-sensor probe and that from image analysis. Here, $W=$ half-width of the total flow duct width in the $x$-direction.

\subsubsection{Benchmark of the Four-Sensor Conductivity Probe}

The four-sensor conductivity probe is benchmarked by a number of images of 
Taylor bubbles with different lengths captured by video camera. The stable Taylor bubbles are generated in the stagnant liquid test column. Three typical images of Taylor bubbles of different lengths are shown in Fig.2.13. From the captured images, the contour of the Taylor bubble is calculated with respect to the slug length assuming symmetric front and flat rear interfaces. According to the images analyzed, the $6^{\text {th }}$ order polynomial is found to best fit all of the interfacial profiles of the captured images.

When the contour function $f(r)$ is determined, the local void fraction and the interfacial area concentration are calculated by (Revankar and Ishii, 1993)

$\alpha(r)=\left(1-\frac{f(r)}{L_{s}}\right) N_{s} \frac{L_{s}}{v_{s}}$,

and

$a_{i}(r)=\left(1+\sqrt{(d f / d r)^{2}+1}\right) \frac{N_{s}}{v_{s}}$,

where $L_{\mathrm{s}}$ is the Taylor bubble chord length, $N_{\mathrm{s}}$ is the number of Taylor bubbles counted by the probe per second, and $v_{\mathrm{s}}$ is the Taylor bubble rise velocity.
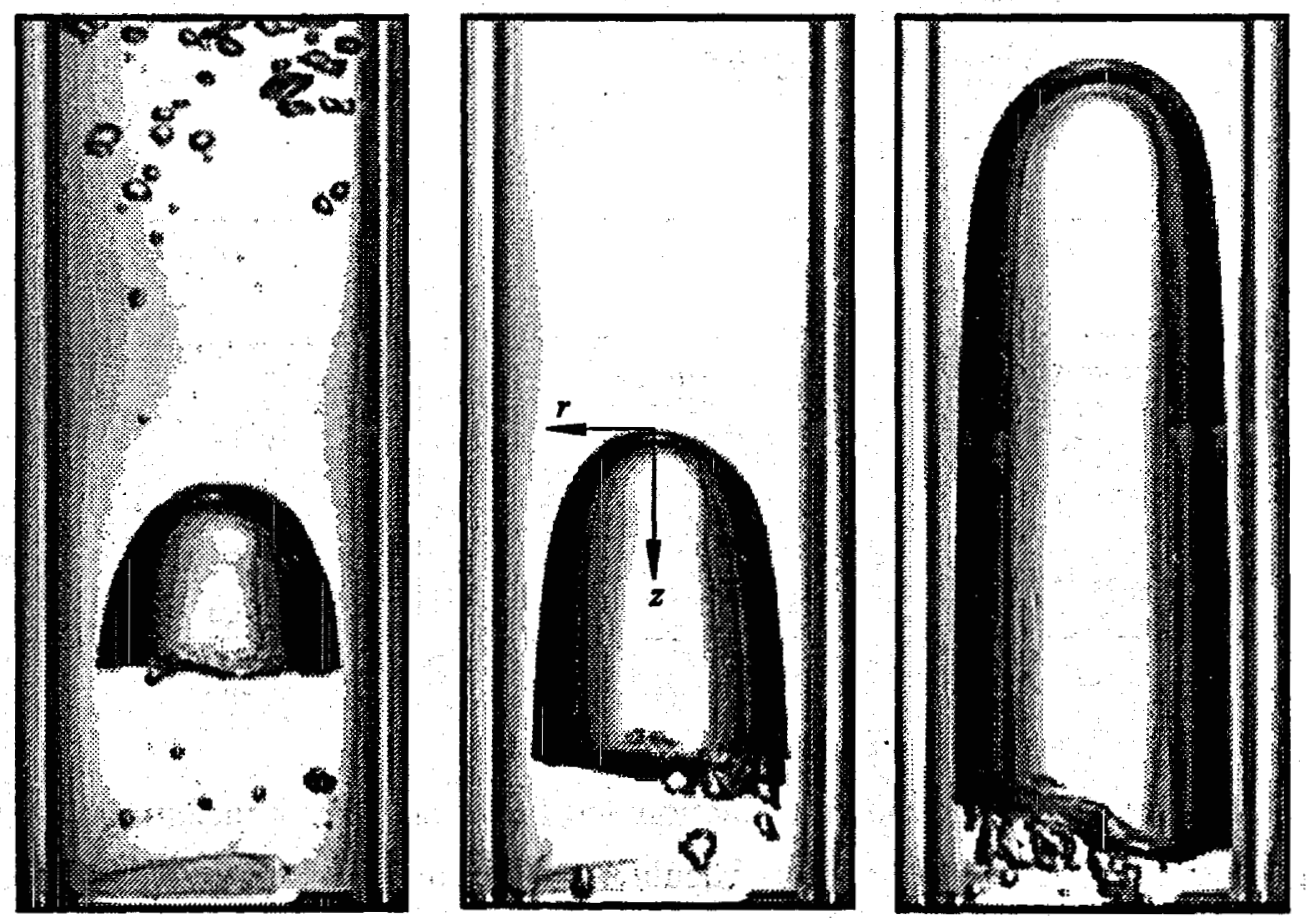

Figure 2.13. Examples of Taylor bubble images captured by CCD camera. The frame size shown here; width $=6.35 \mathrm{~cm}$ and length $=14.55 \mathrm{~cm}$. 
The comparisons between the values calculated based on the image analysis and the ones obtained by the four-sensor probe are shown in Fig.2.14. In the figures, solid line represents the calculated value, and the squares represent the data acquired by the probe. The agreement between the calculated values and the experimental data is quite acceptable in both void fraction and interfacial area concentration comparisons. Here, some deviations between the calculated values and the experimental data can be mainly attributed to the error in estimating the Taylor bubble chord-length. Since there can be variations in Taylor bubble length at given flow conditions, the calculated values based on the average Taylor bubble length can cause significant error. Another reason for deviation can be found from the fact that the image analysis is based on the smooth front and flat rear interfaces of stable Taylor bubbles, while the actual contour of the Taylor bubbles can be highly distorted. Also noted is that the Taylor bubble is assumed to be symmetric when the contour of the Taylor bubble is calculated, whereas the actual Taylor bubble nose deviates from the center.
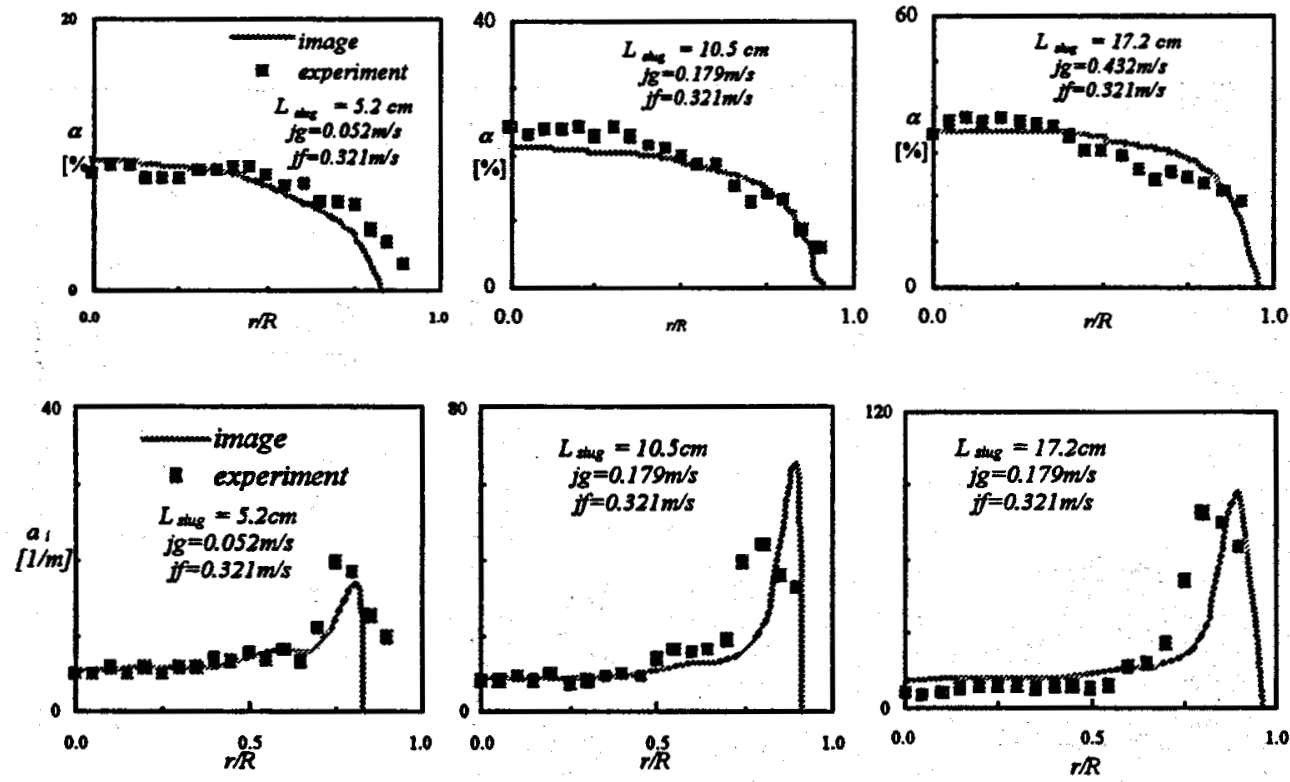

Figure 2.14. Comparison of the void fraction and interfacial area concentration between the experimental data and the values calculated based on the image analysis for flow conditions; $\left\langle j_{p}\right\rangle$ fixed at $0.321 \mathrm{~m} / \mathrm{s}$ and $\left\langle j_{g}\right\rangle$ varied at $(a)\left\langle j_{g}\right\rangle=0.052 \mathrm{~m} / \mathrm{s}$, (b) $\left\langle j_{g}\right\rangle=0.179$ $\mathrm{m} / \mathrm{s}$, and $(\mathrm{c})\left\langle j_{g}>=0.432 \mathrm{~m} / \mathrm{s}\right.$. 
To further check the reliability of the four-sensor conductivity probe measurement, the measured and calculated void fraction and interfacial area concentration are area-averaged and compared with each other. The results are shown in Fig.2.15(a) and (b). In most cases, the agreement is good within $\pm 10 \%$ difference. Thus, accounting for the average effect of the image calculation and the effect of distorted interfaces of the actual Taylor bubbles, it can be concluded that the agreement between the calculated values and the experimental data is good.
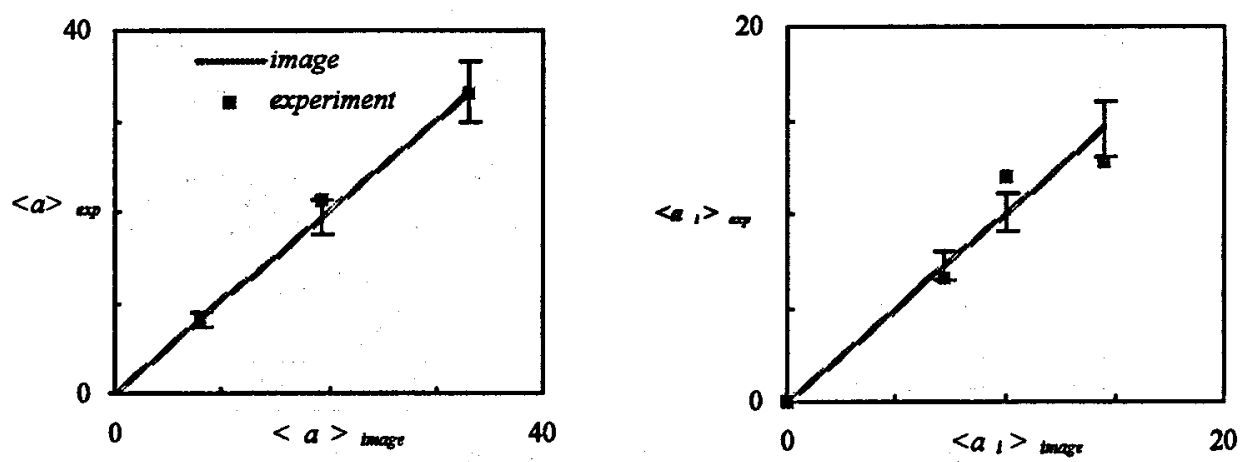

Figure 2.15. Comparison between the area-averaged values of measured and calculated void fraction and interfacial area. Error bars shown here $\pm 10 \%$

\subsubsection{Conductivity and Optical Four-Sensor Probe Benchmark}

The benchmark test between the four-sensor conductivity probe system developed Purdue University and the four-sensor optical probe system developed by French Atomic Energy Commission, France was performed at the Thermal-hydraulics and Reactor Safety Laboratory at Purdue University. Some very useful information on the capabilities of both systems as well as on the main system components influencing the measurement accuracy was obtained. In this chapter, the results of the benchmark test (Le Corre et al., 2000) will be described briefly.

A global comparison between the two independent systems has been first performed in order to check the agreement between the two system measurements. Then, a benchmark coupling methodology has been performed in order to extract and compare the information between each system component (i.e., probe, acquisition, thresholding and processing). Figure 2.16 shows the schematic diagram of the benchmark decomposition. 


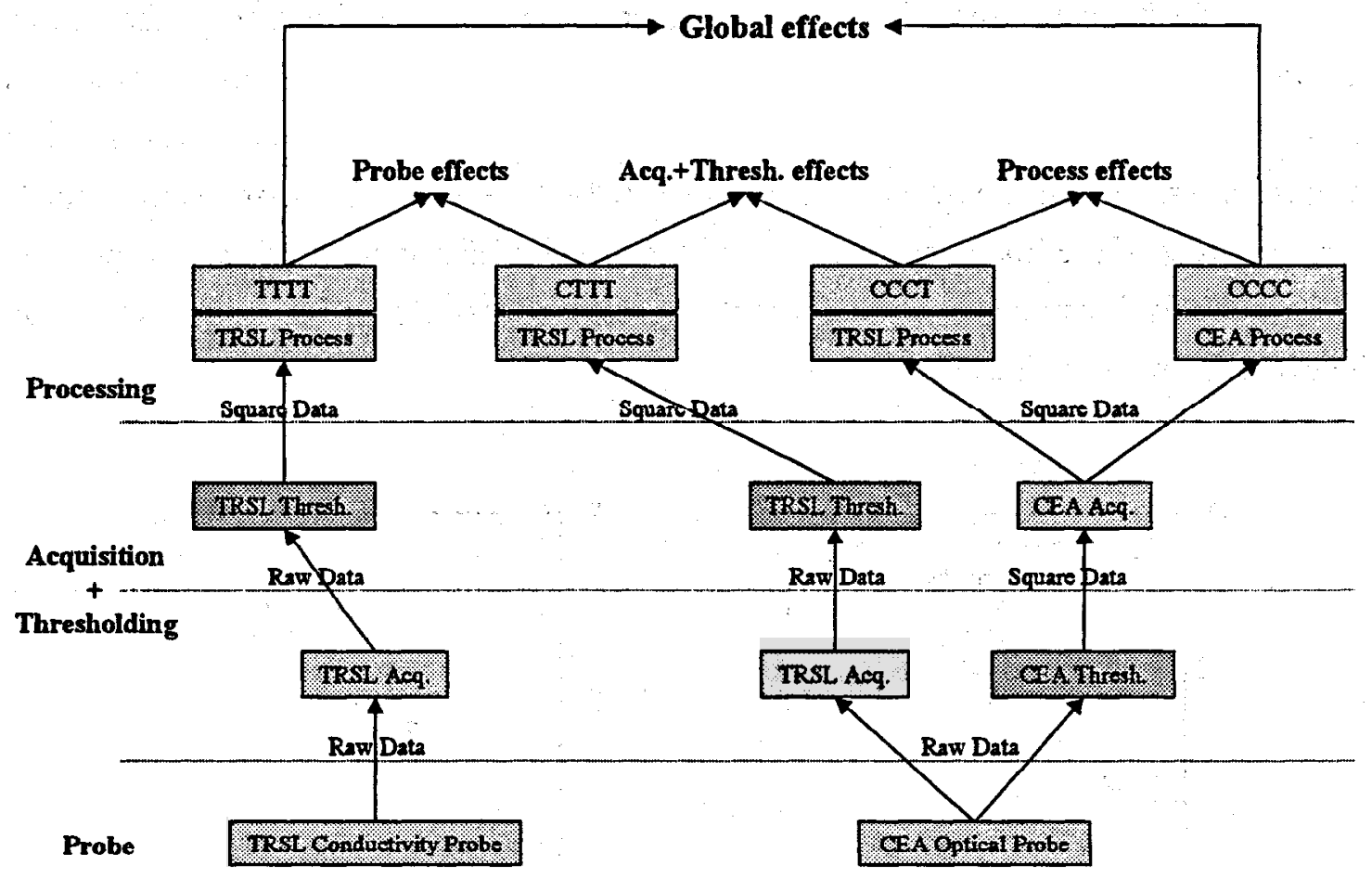

Figure 2.16. Benchmark decomposition.

This methodology allows one to quantify the effect of each component on the two-phase flow parameter measurements. Figure 2.17 shows the system component impacts on two-phase flow parameter measurements. It has been found that the probe effects have the most important impacts on the measurements: therefore it is essential to give a high consideration to the probe design and probe fabrication before using it for two-phase flow parameter measurements. It has also been shown that the acquisition and thresholding effects did not affect the measurements if they were set up properly. Several rules have been suggested in order to minimizing the measurement errors. At last, the interfacial area concentration processing comparisons were not satisfactory; a processing software evaluation using numerical simulations would be useful in order to interpret the phenomena involved in the calculation differences. The overall results of this four-sensor benchmark for the measurements of local time-averaged two-phase flow parameters were encouraging and promising about the high accuracy of the measurement using intrusive multi-sensor probe. However, a benchmark with a non-intrusive method such as image analysis would greatly be useful to confirm the probe measurements. The detailed discussion can be found in the paper (Le Corre et al., 2000). 


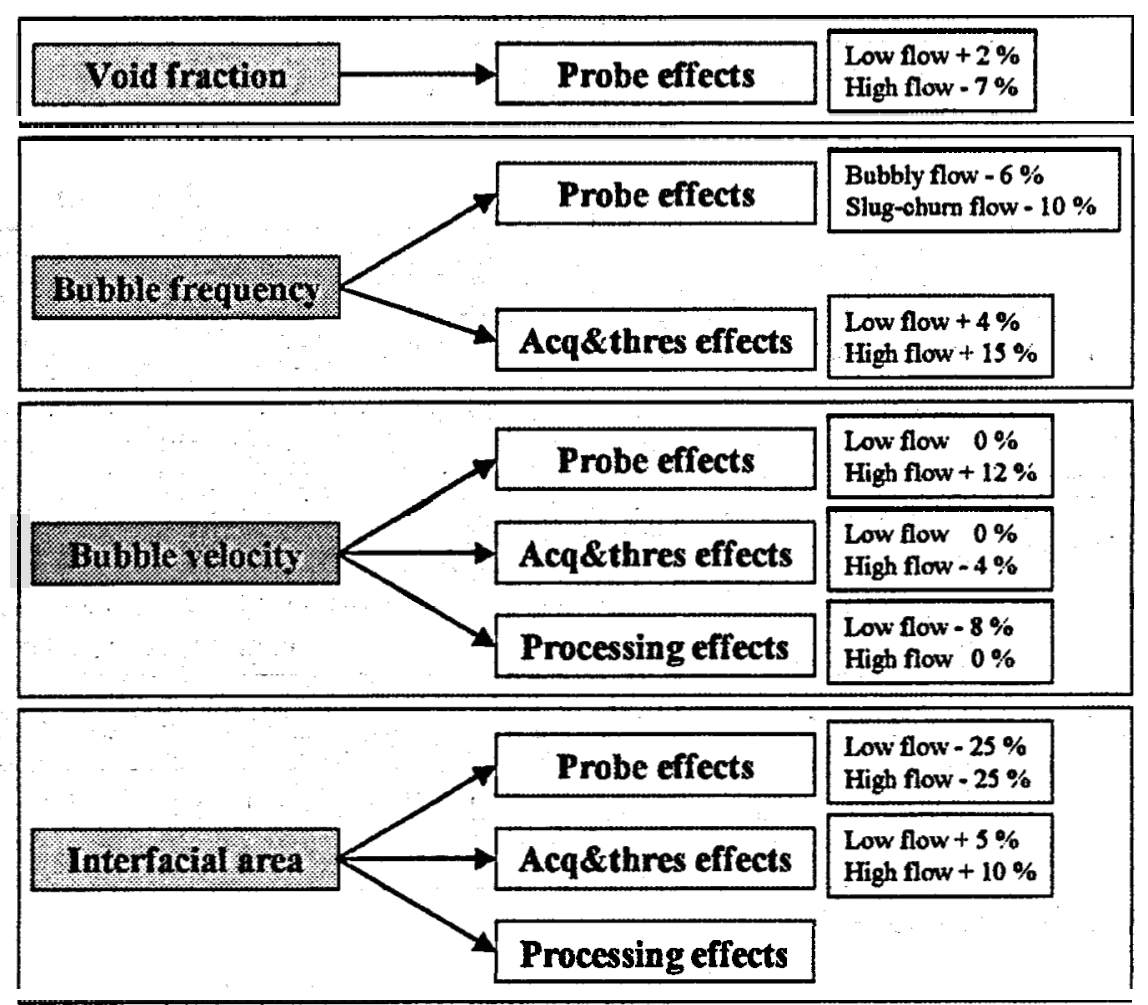

Figure 2.17. System component impacts on two-phase flow parameter measurements.

\subsubsection{Numerical Evaluation and Correction Method for Multi-Sensor Probe Measurement Techniques}

Accurate measurement of local time-averaged two-phase flow parameters (void fraction, bubble velocity and interfacial area concentration) is important for the evaluation of two-phase flow models as well as for the development of closure relations used in the two-fluid model. In this chapter, a numerical simulation of the measurement of these parameters using a multi-sensor probe performed for bubbly flow (Le Corre and Ishii, 2002) will be described briefly.

Figure 2.18 shows the schematic diagram of numerical simulation methodology used in this study. In this study, the double and four-sensor probe instrumentation techniques for the measurement of local axial bubble velocity and local interfacial area concentration were evaluated numerically. In order to emphasize the effect of the probe geometry on the measurement accuracy, a flow with constant bubble velocity was first considered. Then a more general flow with isotropic bubble velocity fluctuations was considered. 


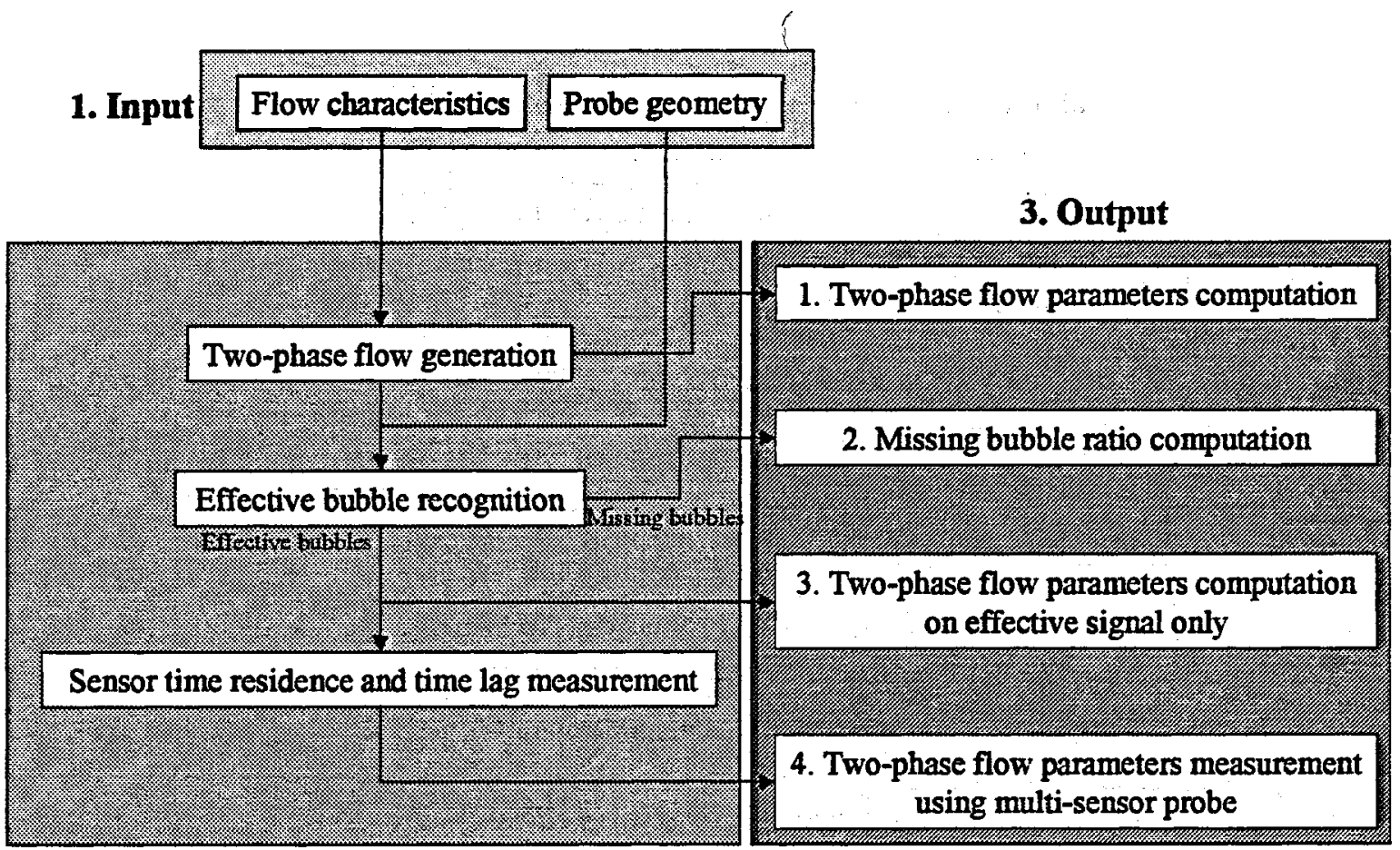

2. Monte-Carlo simulation

Figure 2.18. Numerical simulation methodology.

In the case of axial bubble velocity measurement with a double-sensor probe method (using a statistical average), it was found that a probe design criterion was needed in order to limit the over-estimation of the actual velocity. However, in the case of a flow with high bubble velocity fluctuations, the over-estimation can still go up to $30 \%$ which constitutes a poor accuracy.

In the case of interfacial area concentration measurement using double or four-sensor probe, it was shown that the probe design criterion needed to get a fair accuracy is much less limiting than for the bubble velocity measurement. The accuracy of the double-sensor probe method is fairly good for any flow conditions with spherical bubbles, whereas the four-sensor probe method under-estimates the total interfacial area up to $30 \%$ (after correction), this behavior is due to a higher rate of missing bubbles and also because, in average, a missing bubble transports more interfacial area than an effective bubble. In order to improve the measurement method, a new correction procedure taking accurately into account the interfacial area from the missing bubbles as well as the probe geometrical effects was suggested. The correlations are based on the simulations and it was shown that they do not depend on the probe geometry characteristics nor on the bubble velocity field 
but on the only missing bubble ratio as parameter. The detailed discussion can be found in the paper (Le Corre and Ishii, 2002).

\subsection{Hotfilm Anemometry Methodology}

In this chapter, the hotfilm anemometry probe methodology developed in the Thermal-hydraulics and Reactor Safety Laboratory at Purdue University (Hibiki et al., 1998b) will be explained in detail.

Liquid velocity and turbulent intensity measurements were made using a hotfilm anemometer system developed by TSI Incorporated. The FLOWPOINT system is a fully-integrated, thermal anemometer-based system that measures the local fluid velocity and local fluid temperature. Each system consists of a hotfilm probe, a sample/hold box, a thermocouple sensor, and a FLOWPONT velocity transducer which interfaces with an IBM/PC-XT computer. The system is simple enough to measure a one-dimensional flow, yet it has the ability to measure full three-dimensional flow fields.

A hotfilm probe is shown in Fig.2.19. Each probe is fully manufactured and ready to use out of the box. The probes used in these experiments were the rugged TSI Model $1231 \mathrm{~W}$ designed with a conical tip. The tip had a sensor diameter of 50 microns and a sensor length of $1 \mathrm{~mm}$. Such a small sensor area is able to give nearly a point velocity

\section{Standard 90 Conical Probe - Model 1231W}

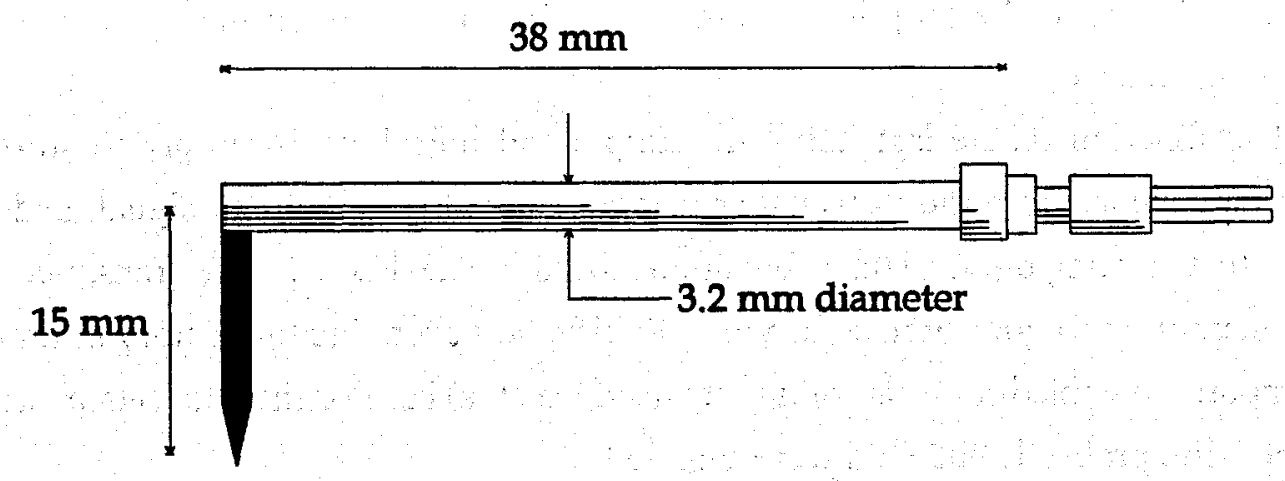

Figure 2.19. Hotfilm conical probe. 
measurement. In the following experiments, the probes were mounted to measure the local liquid velocity in the axial direction. This probe model was used because of its structural integrity in liquid flow and the conical tip's ability to effectively pierce an oncoming bubble, unlike a wedge tip or thin wire. For a conical hot film probe for gas phase, typical upper frequency response in air at $100 \mathrm{~m} / \mathrm{s}$ is $150 \mathrm{kHz}$. For a conical hot film probe for liquid phase, the frequency response of sensor in water is equal to or greater than in air. Actual response levels may be lower due to thermal boundary layer effects.

The main processing unit of the FLOWPOINT system is the velocity transducer. The hotfilm sensor is basically a resistor on a Wheatstone bridge with the resistor's exposed sensor being maintained at a constant temperature. When there is a liquid flow over the sensor, a characteristic amount of heat is convected away from the sensor depending on the temperature difference between the fluid-sensor interface and the local convection coefficient. Thus, the body temperature is lowered. This temperature change in the sensor subsequently changes the sensor's electrical resistance seen by the Wheatstone bridge. When the amplifier senses the off-balance of the bridge, it adjusts the voltage to the top of bridge to keep it in balance. This rapid and changing bridge voltage signal is then characteristic of the local velocity. When the voltage signal is calibrated to the fluid velocity, it represents the time history of the local fluctuating fluid velocity as will be shown later. Since the local fluid temperature also affects the heat transfer from the hotfilm sensor, a thermocouple is placed in the same plane as the hotfilm sensor to measure the local fluid temperature. The assumption is made that the temperature is spatially uniform on each measuring plane and the FLOWPOINT software adjusts the voltage signal accordingly. Typically, the loop is allowed to run for an hour or so until a steady-state temperature is reached.

The function of the last, item, the sample and hold box, is simply to store the analog voltage signal from the Wheatstone bridge, convert it to a digital signal, and then transfer it to the computer's data acquisition board. The FLOWPONT transducer can provide a maximum frequency response of $100 \mathrm{kHz}$, however, due to memory limitations of the computer, the maximum sampling rate used was $5 \mathrm{kHz}$. For the data sets measured with the hot film probe, 15000 data were sampled.

A calibration curve for the hotfilm velocity probe is shown in Fig.2.20. The hotfilm probe was calibrated with a Pitot-static tube, each positioned along the test section centerline. The minimum distance between the hotfilm probe and the Pitot-static tube was limited to thirty pipe diameters. This was due to the inability to mount multiple probes on 
the same flange at the same radial location. Thus, there may have been some flow development considerations. However, the probe was calibrated in a single phase flow, and turbulent flow typically develops between ten to thirty pipe diameters. For the calibration of the hot film probe, radial profiles of the liquid velocity and the void fraction were obtained and then the local superficial liquid velocities were integrated over the area of the pipe to determine the superficial liquid flow velocities. The correction for measured liquid velocities could be corrected by comparing the superficial liquid flow velocity with one measured by a rotameter. The manometer reading from the Pitot-static tube was converted to a velocity measurement and then plotted against the corresponding bridge voltage from the hotfilm. Although, it was mentioned that the hotfilm anemometer does have the advantage of high sensitivity to low fluid velocities, unfortunately the probe cannot be used throughout the low velocity range. This is due to the limitation as to how well a low velocity may be determined experimentally. Unfortunately, the Pitot-static tube does not provide very good accuracy in this region, however, it is the only option for a local velocity measurement.

The typical time history of a hotfilm voltage signal is shown in Fig.2.21. As would be expected for turbulent flow, the voltage is seen to fluctuate around some average value until the probe encounters an air bubble. When a bubble is encountered, the voltage begins to drop rapidly. This is the process which eventually causes the probe burn out mentioned earlier. Since the hotfilm on the probe has encountered slowly circulating air, the convection coefficient for the film decreases by magnitudes causing the temperature of the probe to rise dramatically and quickly. Accordingly, the system compensates by lowering the voltage at the top of the Wheatstone bridge. When the bubble passes completely and the probe is re-wetted, the opposite process occurs. In some instances for the latter case, it is possible to see the overcompensation of the bridge voltage from the upward voltage spike at the end of each passing bubble.

In order to obtain a liquid velocity representative of the flow, it is necessary to filter out the voltage depressions and spikes due to the bubbles hitting and passing the probe, respectively. The voltage spikes are removed from the signal using a threshold scheme. The minimum voltage threshold is typically set near the zero liquid velocity voltage. This is usually the case because the hotfilm anemometer circuitry tends to over compensate the voltage reduction when a bubble suddenly envelopes the tip of the probe. Although the actual voltage change due to the probe encountering the bubble is not important or accurate, the time that the probe is exposed to the bubble can be used to 


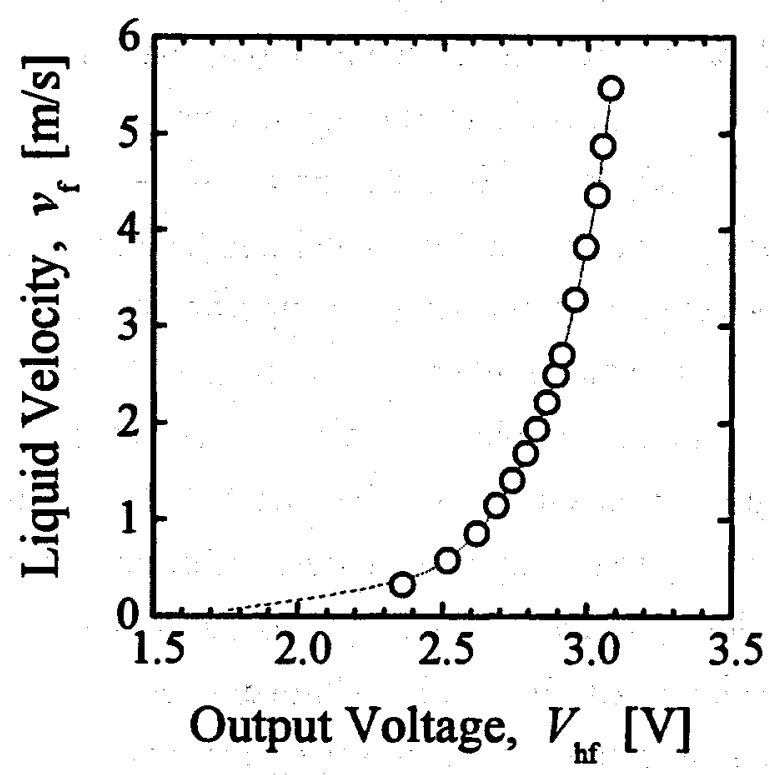

Figure 2.20. Hotfilm calibration curve.

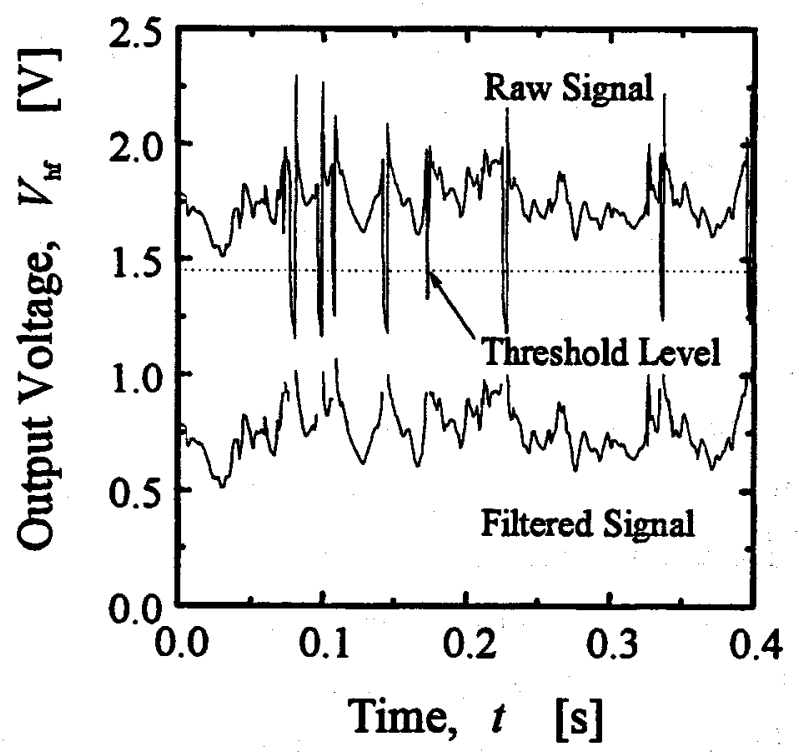

Figure 2.21. Raw and filtered hotfilm probe signal. 
determine the local time-averaged void fraction. Consequently, this information is valuable in that it serves as a cross reference with the void fraction from the conductivity probe, providing more reassurance that the data is reliable.

A bubble-filtered signal is shown in Fig 2.21. It is offset by -1.0 volt from the raw signal above it. To validate the filter used in hotfilm anemometry, void fraction measured by hotfilm anemometry was compared with that measured by the double sensor probe method as shown in Fig.2.22. The void fraction measured by hotfilm anemometry is a little larger than that by the double sensor probe method. The hotfilm anemometer circuitry tends to over compensate the voltage reduction when a bubble suddenly envelopes or passes though the tip of the probe as shown in Fig.2.21. Since the voltage spikes after the passing bubble are also removed by the filter, hotfilm anemometry may overestimate the void fraction. For example, when the sampling frequency is $5 \mathrm{kHz}$ and the velocity of the bubble with a diameter of $3 \mathrm{~mm}$ is $1 \mathrm{~m} / \mathrm{s}, 15$ data points are acquired as the bubble passes through. If one or two data points after the passing bubble are removed by this filter, the hotfilm anemometry overestimates the void fraction on the order of $10-20 \%$. Therefore, the result shown in Fig. 2.22 means the filter used in this study removes the voltage spike due to the passing bubble appropriately. After the bubbles have been removed from the signal, the voltages can be converted to velocities using the calibration curve and the statistical parameters identifying the turbulent flow can be calculated. The parameters used in these experiments are the average liquid velocity defined as

$v_{f}=\frac{\sum_{i=1}^{N} v_{f, i}}{N}$,

where $N$ is the number of velocity samples, and the liquid turbulent fluctuation defined as $v_{f}^{\prime}=\sqrt{\frac{\sum_{i=1}^{N}\left(v_{f, i}-v_{f}\right)^{2}}{N}}$.

Rather than using the turbulent fluctuation, typically the turbulent intensity is reported in the literature as

T.I. $\equiv \frac{v_{f}^{\prime}}{v_{f, \max }}$,

where $v_{f, \max }^{\prime}$ is the maximum average liquid velocity in the radial velocity profile. 


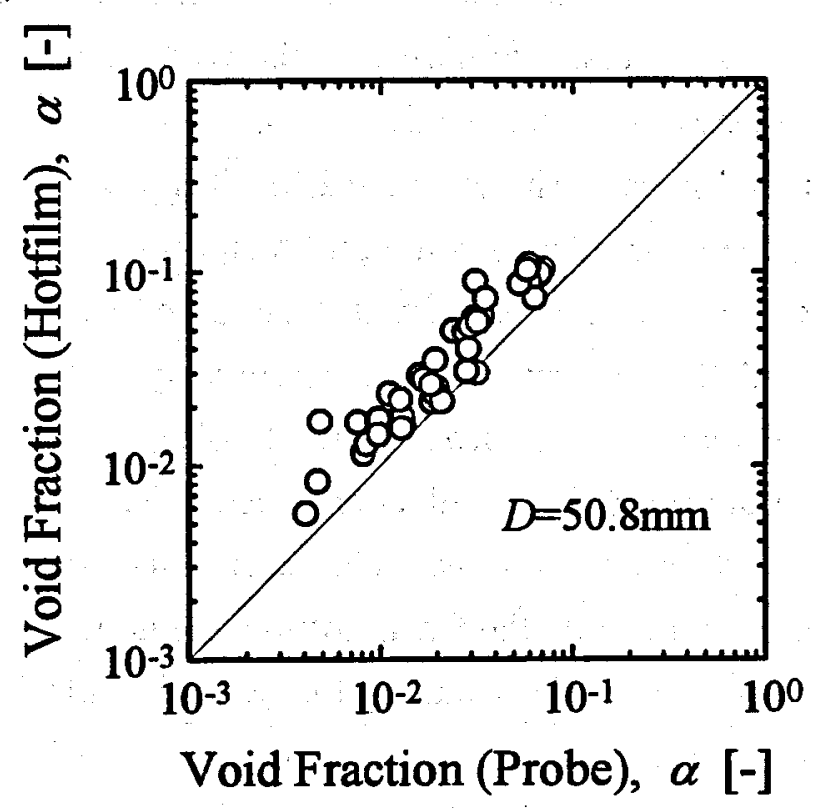

Figure 2.22 Verification of filter used in hotfilm anemometry by void fraction data. 


\section{TWO-GROUP INTERFACIAL AREA TRANSPORT EQUATIONS}

\subsection{Fluid Particle Number Density Transport Equation}

The transport equations for the interfacial area concentration and void fraction can be developed from the fluid particle number density transport equation analogous to Boltzmann's transport equation (Reyes, 1989; Kocamustafaogullari and Ishii, 1995). The fluid particle number density transport equation of particles having volume, $v$, is deduced from a simple procedure accounting for the fluid particles entering and leaving a control volume through different mechanisms (Kocamustafaogullari and Ishii, 1995):

$$
\frac{\partial f}{\partial t}+\nabla \cdot\left(f \overrightarrow{v_{p}}\right)=\sum_{j=1}^{4} s_{j}+s_{p h} \text {, }
$$

where $f(x, v, t)$ is the particle density distribution function, which is assumed to be continuous and specifies the probable number density of fluid particles at a given time, $t$, whose centers lie in the spatial range, $d \vec{x}$, about a position, $\vec{x}$, with particle volumes between $v$ and $v+d v ; \overrightarrow{v_{p}}(\vec{x}, v, t)$ is the particle velocity of volumes between $v$ and $v+d v$ at time, $t$, in the spatial range, $d x$, about a position, $x . s_{\mathrm{ph}}$ is the fluid particle sink or source rate due to the phase change. For example, for one component bubbly flow, $s_{\mathrm{ph}}$ represents the bulk liquid bubble nucleation rate due to the homogeneous and heterogeneous nucleation, and the collapse rate due to condensation for the subcooled boiling flow. The significance and methods evaluating $s_{\mathrm{ph}}$ are discussed in great detail by Kocamustafaogullari and Ishii (1983). The wall nucleation rate which is not included in $S_{\mathrm{ph}}$ must be specified as a boundary condition. The interaction term, $\sum_{j=1}^{4} s_{j}$, represents the net rate of change in the number density distribution function, $f$, due to the particle breakup and coalescence processes. In essence, it serves as source and/or sink terms for fluid particles for two-component dispersed two-phase flow. $s_{1}$ and $s_{2}$, respectively, represent the formation and loss rates of particles of volume, $v$, per unit volume of mixture due to breakup. $s_{3}$ and $s_{4}$ represent the rate of formation and loss of particles of size, $v$, due to coalescence. These terms are detailed in the paper presented by Kocamustafaogullari and 
Ishii (1995). It should be noted here that the sink and source terms due to the interfacial stretching and changes in interfacial shapes are not taken into account in this study.

Since the primary focus for most flow studies is on the average fluid particle behavior, the fluid particle number density transport equation of particles averaged over all particle sizes from the minimum particle volume to the maximum possible particle volume. Thus, the one-group fluid particle number density transport equation is obtained as (Kocamustafaogullari and Ishii, 1995):

$\frac{\partial n}{\partial t}+\nabla \cdot n \overline{v_{p m}}=\sum_{j} \int_{v_{\min }}^{v_{\max }} s_{j} d v+\int_{v_{\min }}^{v_{\max }} s_{p h} d v=\sum_{j} S_{j}+S_{p h}$

where $n(\vec{x}, t)$ and $\vec{v}_{p m}$ denote the number density of particles of all sizes and the average local particle velocity weighted by the particle number as defined by Eq.(3.3) and Eq.(3.4), respectively.

$$
\begin{aligned}
& n(\vec{x}, t) \equiv \int_{v_{\min }}^{v_{\max }} f(\vec{x}, v, t) d v, \\
& \overrightarrow{v_{p m}}(\vec{x}, t) \equiv \frac{\int_{v_{\min }}^{v_{\max }} f(\vec{x}, v, t) \overrightarrow{v_{p}}(\vec{x}, v, t) d v}{\int_{v_{\min }}^{v_{\max }} f(\vec{x}, v, t) d v} .
\end{aligned}
$$

The one-group interfacial area transport equation would be sufficient to describe the interfacial area transport mechanism for the case of uniform bubble size distribution such as a bubbly flow system with low void fraction where no cap bubbles appear. However, as coalescence dominates the system behavior and cap bubbles are formed, another group must be considered (Uhle et al., 1998). The mechanism of bubble breakup and coalescence in cap bubbly flow or slug flow can be quite different from that in bubbly flow. The mechanism for coalescence in which the cap bubbles overtake the smaller diameter bubbles or entrain them in their wakes represents the dominating phenomena by which slug flow is produced. In order to model the integral sink and source terms in Eq.(3.2) caused by particle coalescence and breakup, a general approach treats the bubbles in two groups, which are the spherical/distorted bubble group (Group 1) and the cap/slug bubble group (Group II) (Ishii and Kojasoy, 1993; Wu et al., 1998a). These two groups would result in two fluid particle number density transport equations. The one-group fluid particle number density transport equation can be divided into the two-group fluid particle number density transport equations as: 


$$
\begin{aligned}
& \frac{\partial n_{1}}{\partial t}+\nabla \cdot n_{1} \overrightarrow{v_{p m, 1}}=\sum_{j}\left(S_{j, 1}+S_{j, 12}\right)+\left(S_{p h, 1}+S_{p h, 12}\right), \\
& \frac{\partial n_{2}}{\partial t}+\nabla \cdot n_{2} \overline{v_{p m, 2}}=\sum_{j}\left(S_{j, 2}+S_{j, 21}\right)+\left(S_{p h, 2}+S_{p h, 21}\right),
\end{aligned}
$$

where $n_{\mathrm{k}}(\vec{x}, t)$ and $\overrightarrow{v_{p m, k}}$ denote the number density of particles in the $k$-group of all sizes in a given group and the average local particle velocity weighted by the particle number in the $k$-group as defined by Eq.(3.7) and Eq.(3.8), respectively.

$$
\begin{aligned}
& n_{k}(\vec{x}, t) \equiv \int_{v_{\min , k}}^{v_{\max , k}} f(\vec{x}, v, t) d v, \\
& \overrightarrow{v_{p m, k}}(\vec{x}, t) \equiv \frac{\int_{v_{\max k}}^{v_{\max }} f(\vec{x}, v, t) \overrightarrow{v_{p}}(\vec{x}, v, t) d v}{\int_{v_{\min , k}}^{v_{\max , k}} f(\vec{x}, v, t) d v} .
\end{aligned}
$$

In the two-group transport equations, there are some inter-group particle exchange terms, $S_{\mathrm{j}, 12}$ and $S_{\mathrm{j}, 21}$, which need to be modeled explicitly.

Uhle et al (1998) suggested that a two-group model would be sufficient to characterize the transition from the bubbly through annular to the dispersed droplet flow regimes. For example, in the annular flow regime, the annular flow can be treated as "slug flow" where the "slug" is very long such that the tail and the head have no contribution. To reach this stage, coalescence rate is extremely high in the region of annular flow transition.

\subsection{Fluid Particle Interfacial Area Concentration Transport Equation}

In the two-fluid model, the parameter of interest is the interfacial area, $a_{i}$, rather than the bubble number density. To obtain the transport equations for interfacial area concentration, Eqs.(3.5) and (3.6) can be modified with the following geometric relation (Wu et al., 1998b):

$n_{k}=\frac{\alpha_{k}}{V_{b, k}}=\psi_{k}\left(\frac{a_{i, k}^{3}}{\alpha_{k}^{2}}\right)$

where $\alpha, V_{b}$, and $\psi$ denote the void fraction, the average bubble volume, and the factor depending on the shape of the bubbles, respectively. The subscript, $k$, specifies the bubble group. For spherical bubbles, $\psi_{1}$ equals $1 /(36 \pi)$, whereas for cap bubbles with the shape of semi-sphere, $\psi_{2}$ is $4 /(243 \pi)$. The void fraction and the interfacial area concentration are defined by 
$\alpha_{k}(\bar{x}, t) \equiv \int_{v_{\min , x}}^{v_{\max , t}} f(\vec{x}, v, t) v d v$,

$a_{i, k}(\vec{x}, t) \equiv \int_{v_{\min , k}}^{v_{\max , k}} f(\vec{x}, v, t) A_{i, k}(v) d v$,

where $A_{\mathrm{i}, \mathrm{k}}(v)$ is the average interfacial area of the $k$-group particles of volume $v$, which is independent of the spatial coordinate system. Substituting Eq.(3.9) into Eqs.(3.5) and (3.6) yields:

$\frac{\partial a_{i, 1}}{\partial t}+\nabla \cdot a_{i, 1} \overline{v_{i, 1}}=\frac{1}{3 \psi_{1}}\left(\frac{\alpha_{1}}{a_{i, 1}}\right)^{2}\left[\sum_{j}\left(S_{j, 1}+S_{j, 12}\right)+\left(S_{p h, 1}+S_{p h, 12}\right)\right]+\left(\frac{2 a_{i, 1}}{3 \alpha_{1}}\right)\left[\frac{\partial \alpha_{1}}{\partial t}+\nabla \cdot \alpha_{1} \overrightarrow{v_{i, 1}}\right]$

$\frac{\partial a_{i, 2}}{\partial t}+\nabla \cdot a_{i, 2} \overline{v_{i, 2}}=\frac{1}{3 \psi_{2}}\left(\frac{\alpha_{2}}{a_{i, 2}}\right)^{2}\left[\sum_{j}\left(S_{j, 2}+S_{j, 21}\right)+\left(S_{p h, 2}+S_{p h, 21}\right)\right]+\left(\frac{2 a_{i, 2}}{3 \alpha_{2}}\right)\left[\frac{\partial \alpha_{2}}{\partial t}+\nabla \cdot \alpha_{2} \overrightarrow{v_{i, 2}}\right]$.

where $\overrightarrow{v_{i, k}}$ denotes the interfacial velocity in the $k$-group as defined by Eq.(3.14).

$\overrightarrow{v_{i, k}}(\vec{x}, t) \equiv \frac{\int_{v_{\min , k}}^{v_{\max , k}} f(\vec{x}, v, t) A_{i, k}(v) \overrightarrow{v_{p}}(\vec{x}, v, t) d v}{\int_{v_{\min , k}}^{v_{\max , k}} f(\vec{x}, v, t) A_{i, k}(v) d v}$

The simplest form of the two-group interfacial area transport equations is the one-dimensional formulation obtained by averaging Eqs.(3.12) and (3.13) over cross-sectional area as:

$$
\begin{aligned}
& \frac{\partial\left\langle a_{i, 1}\right\rangle}{\partial t}+\frac{d}{d z}\left(\left\langle a_{i, 1}\right\rangle\left\langle\left\langle v_{i, 1}\right\rangle\right\rangle_{a}\right)=\frac{1}{3 \psi_{1}}\left(\frac{\left\langle\alpha_{1}\right\rangle}{\left\langle a_{i, 1}\right\rangle}\right)^{2}\left[\sum_{j}\left(\left\langle S_{J, 1}\right\rangle+\left\langle S_{j, 12}\right\rangle\right)+\left(\left\langle S_{p h, 1}\right\rangle+\left\langle S_{p h, 12}\right\rangle\right)\right] \\
& +\left(\frac{2\left\langle a_{i, 1}\right\rangle}{3\left\langle\alpha_{1}\right\rangle}\right)\left[\frac{\partial\left\langle\alpha_{1}\right\rangle}{\partial t}+\frac{d}{d z}\left(\left\langle\alpha_{1}\right\rangle\left\langle\left\langle v_{t, 1}\right\rangle\right\rangle\right)\right]
\end{aligned}
$$

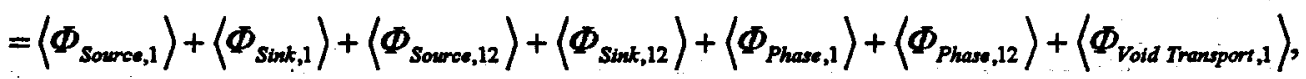

$$
\begin{aligned}
& \left\langle\Phi_{\text {Sorroos, } 1}\right\rangle \equiv \frac{1}{3 \psi_{1}}\left(\frac{\left\langle\alpha_{1}\right\rangle}{\left\langle a_{1,1}\right\rangle}\right)^{2}\left[\left\langle S_{1,1}\right\rangle+\left\langle S_{3,1}\right\rangle\right],\left\langle\Phi_{\text {Sint }, 1}\right\rangle \equiv \frac{1}{3 \psi_{1}}\left(\frac{\left\langle a_{1}\right\rangle}{\left\langle a_{i, 1}\right\rangle}\right)^{2}\left[\left\langle S_{2,1}\right\rangle+\left\langle S_{4,1}\right\rangle\right],
\end{aligned}
$$




$$
\begin{aligned}
& \left\langle\Phi_{\text {Sowrea, }, 2}\right\rangle \equiv \frac{1}{3 \psi_{1}}\left(\frac{\left\langle\alpha_{1}\right\rangle}{\left\langle a_{i, 1}\right\rangle}\right)^{2}\left[\left\langle S_{1,12}\right\rangle+\left\langle S_{3,12}\right\rangle\right], \quad\left\langle\Phi_{\text {Sint, }, 2}\right\rangle \equiv \frac{1}{3 \psi_{1}}\left(\frac{\left\langle\alpha_{1}\right\rangle}{\left\langle a_{i, 1}\right\rangle}\right)^{2}\left[\left\langle S_{2,12}\right\rangle+\left\langle S_{4,12}\right\rangle\right] \\
& \left\langle\Phi_{\text {Phase, } 1}\right\rangle=\frac{1}{3 \psi_{1}}\left(\frac{\left\langle\alpha_{1}\right\rangle}{\left\langle a_{i, 1}\right\rangle}\right)^{2}\left\langle S_{p h, 1}\right\rangle,\left\langle\Phi_{\text {Phass, 12 }}\right\rangle=\frac{1}{3 \psi_{1}}\left(\frac{\left\langle\alpha_{1}\right\rangle}{\left\langle a_{i, 1}\right\rangle}\right)^{2}\left\langle S_{p h, 12}\right\rangle \text {, } \\
& \left\langle\Phi_{\text {Votd Transporr, } 1}\right\rangle \equiv\left(\frac{2\left\langle a_{i, 1}\right\rangle}{3\left\langle\alpha_{1}\right\rangle}\right)\left[\frac{\partial\left\langle\alpha_{1}\right\rangle}{\partial t}+\frac{d}{d z}\left(\left\langle\alpha_{1}\right\rangle\left\langle\left\langle v_{t z, 1}\right\rangle\right)\right)\right] \\
& \frac{\partial\left\langle a_{i, 2}\right\rangle}{\partial t}+\frac{d}{d z}\left(\left\langle a_{i, 2}\right\rangle\left\langle\left(v_{i, 2}\right\rangle\right\rangle_{a}\right)=\frac{1}{3 \psi_{2}}\left(\frac{\left\langle a_{2}\right\rangle}{\left\langle a_{i, 2}\right\rangle}\right)^{2}\left[\sum_{j}\left(\left\langle S_{j, 2}\right\rangle+\left\langle S_{j, 21}\right\rangle\right)+\left(\left\langle S_{p h, 2}\right\rangle+\left\langle S_{p h, 21}\right\rangle\right)\right] \\
& +\left(\frac{2\left\langle a_{i, 2}\right\rangle}{3\left\langle\alpha_{2}\right\rangle}\right)\left[\frac{\partial\left\langle\alpha_{2}\right\rangle}{\partial t}+\frac{d}{d z}\left(\left\langle\alpha_{2}\right\rangle\left\langle\left\langle v_{i s, 2}\right\rangle\right)\right)\right] \\
& =\left\langle\Phi_{\text {Source, } 2}\right\rangle+\left\langle\Phi_{\text {Sink ,2 }}\right\rangle+\left\langle\Phi_{\text {Source, } 21}\right\rangle+\left\langle\Phi_{\text {Sink ,21 }}\right\rangle+\left\langle\Phi_{\text {Phase, } 2}\right\rangle+\left\langle\Phi_{\text {Phase , 21 }}\right\rangle+\left\langle\Phi_{\text {Votd Irenspor , 2 }}\right\rangle \text {, } \\
& \left\langle\Phi_{\text {Source }, 2}\right\rangle \equiv \frac{1}{3 \psi_{2}}\left(\frac{\left\langle\alpha_{2}\right\rangle}{\left\langle a_{i, 2}\right\rangle}\right)^{2}\left[\left\langle S_{1,2}\right\rangle+\left\langle S_{3,2}\right\rangle\right], \quad\left\langle\Phi_{\text {Sink }, 2}\right\rangle \equiv \frac{1}{3 \psi_{2}}\left(\frac{\left\langle\alpha_{2}\right\rangle}{\left\langle a_{i, 2}\right\rangle}\right)^{2}\left[\left\langle S_{2,2}\right\rangle+\left\langle S_{4,2}\right\rangle\right] \\
& \left\langle\Phi_{\text {Source, }, 21}\right\rangle \equiv \frac{1}{3 \psi_{2}}\left(\frac{\left\langle a_{2}\right\rangle}{\left\langle a_{i, 2}\right\rangle}\right)^{2}\left[\left\langle S_{1,21}\right\rangle+\left\langle S_{3,21}\right\rangle\right],\left\langle\Phi_{\text {Sink }, 21}\right\rangle=\frac{1}{3 \psi_{2}}\left(\frac{\left\langle a_{2}\right\rangle}{\left\langle a_{i, 2}\right\rangle}\right)^{2}\left[\left\langle S_{2,21}\right\rangle+\left\langle S_{4,21}\right\rangle\right], \\
& \left\langle\Phi_{\text {Phase }, 2}\right\rangle \equiv \frac{1}{3 \psi_{2}}\left(\frac{\left\langle\alpha_{2}\right\rangle}{\left\langle a_{i, 2}\right\rangle}\right)^{2}\left\langle S_{p h, 2}\right\rangle, \quad\left\langle\Phi_{\text {Phase }, 21}\right\rangle \equiv \frac{1}{3 \psi_{2}}\left(\frac{\left\langle\alpha_{2}\right\rangle}{\left\langle a_{i, 2}\right\rangle}\right)^{2}\left\langle S_{p h, 21}\right\rangle \text {, } \\
& \left\langle\Phi_{\text {Void Transpor, }, 2}\right\rangle \equiv\left(\frac{2\left\langle a_{i, 2}\right\rangle}{3\left\langle\alpha_{2}\right\rangle}\right)\left[\frac{\partial\left\langle\alpha_{2}\right\rangle}{\partial t}+\frac{d}{d z}\left(\left\langle\alpha_{2}\right\rangle\left\langle\left\langle v_{t z, 2}\right\rangle\right\rangle\right)\right] \text {, }
\end{aligned}
$$

where the brackets of $\left\langle>,<<>\right.$, and $\langle<>\rangle_{\text {a }}$ mean the cross-sectional area averaged quantity, the void fraction weighted cross-sectional area averaged quantity, and the interfacial area concentration weighted cross-sectional area averaged quantity, respectively. $z$-direction is taken in the axial flow direction. In Eqs.(3.15) and (3.16), $\left\langle\Phi_{\text {Void }}\right.$ Transport, $k$ includes the interfacial area concentration change due to void fraction transport along the flow direction by the pressure reduction as well as the interchange of void fraction between groups I and II.

The exact mathematical expressions for the area-averaged source and sink terms would involve many covariances that may further complicate the one-dimensional problem. Wu et al. (1998b) discussed the one-dimensional problem due to the covariances as follows. Since these local terms were originally obtained from a finite volume element of the mixture, the functional dependence of the area-averaged sink and source terms on the 
averaged parametirs should be approximately the same if the hydraulic diameter of the flow path is considered as the length scale of the finite element. Therefore, local sink and source terms to be derived in the next section with the parameters averaged within the cross-sectional area are still applicable for the area-averaged source and sink terms in Eqs.(3.15) and (3.16).

Under adiabatic and steady conditions, there are no effects of phase change; thus:

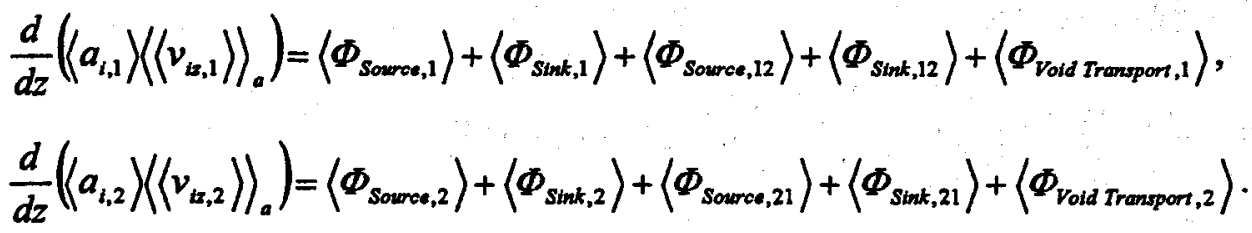

With this approach, the two interfacial area transport equations introduce four additional area-averaged variables such as $\left\langle a_{\mathrm{i}, 1}\right\rangle,\left\langle a_{\mathrm{i}, 2}\right\rangle,\left\langle\alpha_{1}\right\rangle$, and $\left\langle\alpha_{2}\right\rangle$ to the two-fluid model (Ishii et al., 1998; Wu et al., 1998a; Uhle et al., 1998). To close the two-fluid model, Eqs.(3.15) and (3.16) need four more closure equations as (a) overall gas-phase mass conservation, Eq.(3.19), (b) mass balance of the group-I, Eq.(3.20), (c) overall gas-phase momentum conservation, Eq.(3.21), and (d) the velocity difference between the group-I and II bubbles, Eq.(3.22). The velocity difference will be specified by a correlation, $F$. This is similar to the drift flux approach (Uhle et al., 1998).

$\left\langle\alpha_{1}\right\rangle+\left\langle\alpha_{2}\right\rangle=\langle\alpha\rangle$,

$\frac{\partial \rho_{g}\left\langle\alpha_{1}\right\rangle}{\partial t}+\frac{d}{d z}\left(\rho_{g}\left\langle\alpha_{1}\right\rangle\left\langle\left\langle v_{z, 1}\right\rangle\right)\right)=\frac{\rho_{g}}{\psi_{1}}\left(\frac{\left\langle\alpha_{1}\right\rangle}{\left\langle a_{i, 1}\right\rangle}\right)^{3}\left[\sum_{j}\left(\left\langle S_{j, 12}\right\rangle\right)+\left\langle S_{p h, 1}\right\rangle\right]$,

$\left\langle\alpha_{1}\right\rangle\left\langle\left\langle v_{z, 1}\right\rangle\right\rangle+\left\langle\alpha_{2}\right\rangle\left\langle\left\langle v_{z, 2}\right\rangle\right\rangle=\langle\alpha\rangle\left\langle\left\langle v_{z}\right\rangle\right\rangle$,

$\left\langle\left\langle v_{2,2}\right\rangle\right\rangle-\left\langle\left\langle v_{2,1}\right\rangle\right\rangle=F\left(\alpha_{1}, \alpha_{2}, a_{i, 1}, a_{i, 2}\right.$, Properties $)$.

where $v_{z, k}$ is the $z$ component of $\overrightarrow{v_{k}}$, which denotes the average velocity of the center of volume of the dispersed phase in the $k$-group as defined by Eq.(3.23).

$\overrightarrow{v_{k}}(\vec{x}, t) \equiv \frac{\int_{v_{\max }}^{v_{\max }} f(\hat{x}, v, t) v \overrightarrow{v_{p}}(\hat{x}, v, t) d v}{\int_{v_{\min k}}^{v_{\max , k}} f(\vec{x}, v, t) v d v}$ 
45

Under the adiabatic and steady conditions, the transient and phase change terms in Eq.(3.20) are dropped, resulting in:

$$
\begin{aligned}
& \frac{d}{d z}\left(\left\langle\alpha_{1}\right\rangle\left\langle\left\langle v_{z, 1}\right\rangle\right)\right)=\frac{1}{\psi_{1}}\left(\frac{\left\langle\alpha_{1}\right\rangle}{\left\langle a_{i, 1}\right\rangle}\right)^{3} \sum_{j}\left(\left\langle S_{J, 12}\right\rangle\right)-\frac{\left\langle\alpha_{1}\right\rangle\left\langle\left\langle v_{z, 1}\right\rangle\right\rangle}{\rho_{g}} \frac{d \rho_{g}}{d z} \\
& =\left\langle\Psi_{\text {Source, 12 }}\right\rangle+\left\langle\Psi_{\text {Sink ,12 }}\right\rangle+\left\langle\Psi_{\text {fold Transport, , }}\right\rangle \text {, } \\
& \left\langle\Psi_{\text {source, }, 12}\right\rangle \equiv \frac{1}{\psi_{1}}\left(\frac{\left\langle\alpha_{1}\right\rangle}{\left\langle a_{i, 1}\right\rangle}\right)^{3}\left(\left\langle S_{1,12}\right\rangle+\left\langle S_{3,12}\right\rangle\right),\left\langle\Psi_{\operatorname{sink}, 12}\right\rangle \equiv \frac{1}{\psi_{1}}\left(\frac{\left\langle\alpha_{1}\right\rangle}{\left\langle a_{i, 1}\right\rangle}\right)^{3}\left(\left\langle S_{2,12}\right\rangle+\left\langle S_{4,12}\right\rangle\right) \\
& \left\langle\Psi_{\text {Void Transport, }, 1}\right\rangle \equiv-\frac{\left\langle\alpha_{1}\right\rangle\left\langle\left\langle v_{z, 1}\right\rangle\right\rangle}{\rho_{g}} \frac{d \rho_{g}}{d z} \text {. }
\end{aligned}
$$




\section{MODEL DEVELOPMENT OF BUBBLE COALESCENCE AND BREAKUP}

\subsection{Classification of Interfacial Area Transport Mechanisms \\ Due to Bubble Coalescence and Breakup}

To model the integral source and sink terms in Eqs.(3.15) and (3.16) caused by bubble coalescence and breakup, the possible combinations of bubble interactions can be classified into eight categories in terms of the belonging bubble group (see Fig.4.1): (1) the coalescence of bubbles (Group I) into a bubble (Group I), (2) the breakup of a bubble (Group I) into bubbles (Group I), (3) the coalescence of bubbles (Group I and II) into a bubble (Group II), (4) the breakup of a bubble (Group II) into bubbles (Group I and II), (5) the coalescence of bubbles (Group I) into a bubble (Group II), (6) the breakup of a bubble (Group II) into bubbles (Group I), (7) the coalescence of bubbles (Group II) into a bubble (Group II), and (8) the breakup of a bubble (Group II) into bubbles (Group II) (Hibiki and Ishii, 2000b). As summarized in Table 4.1, the mechanisms of bubble coalescence and breakup would be mainly divided into four categories: (1) the coalescence due to random collisions driven by turbulence, (2) the coalescence due to wake entrainment, (3) the breakup upon the impact of turbulent eddies, and (4) the breakup of large cap bubbles due to flow instability on the bubble surface.

In the first stage of a theoretical study on the interfacial area transport phenomena, bubbly flow was the focus (Ishii et al., 1997; Wu et al., 1997a; 1998b; Hibiki and Ishii, 1999b; 2000a). For the flow region where no cap and slug bubbles appear, the two-group interfacial area transport equations could be reduced to one group. The sink and source terms in the one-group interfacial area transport equation should be properly modeled based on probable mechanisms of bubble coalescence and breakup. Collisions between bubbles may occur due to (a) the turbulent motion of the liquid, (b) differences in bubble rise velocities and (c) the effect of liquid shear. However, Prince and Branch (1990) found that the main influence on bubble coalescence was the velocity fluctuations of the liquid. In particular, the higher the liquid phase turbulence level, the more likely it is that the liquid eddies will break up individual bubbles (Millies et al., 1996). In the previous study of the present authors (Hibiki and Ishii, 1999b; 2000a), the main mechanisms of bubble coalescence and breakup were successfully modeled as the coalescence due to random 


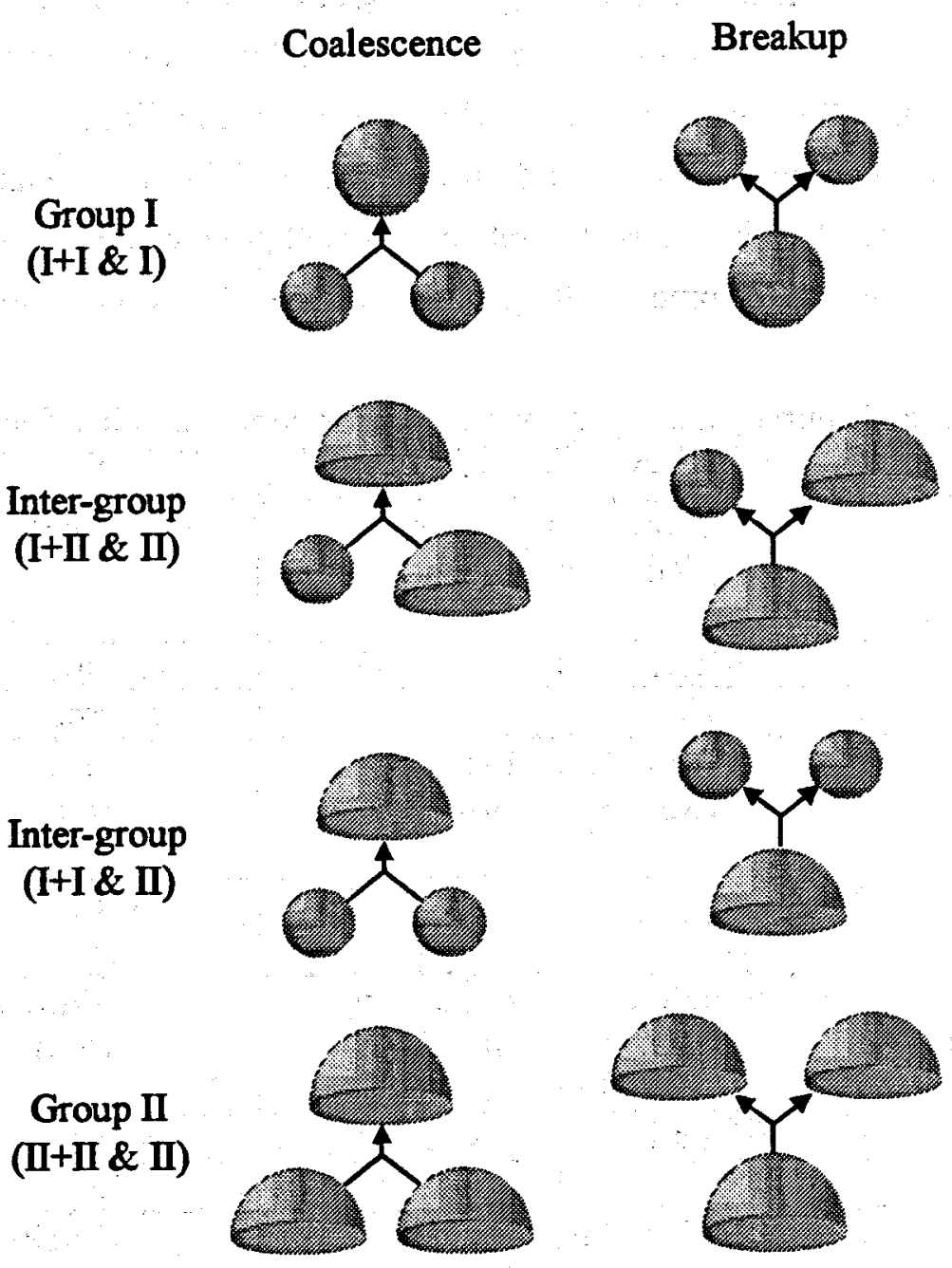

Figure 4.1. Classification of possible interactions of two-group bubbles.

collisions driven by turbulence and the breakup upon the impact of turbulent eddies. In the study, the interactions between the two groups as well as sink and source terms in the group II were omitted. The modeled interfacial area transport equation could fit interfacial area concentrations of bubbly flows measured in round pipes within the average relative deviation of $\pm 11.6 \%$ (Hibiki and Ishii, 1999b; 2000a).

As the next stage, bubbly-to-slug flow transition will be the focus in this study. The two-group model will be developed with the necessary inter-group coupling terms due to turbulent impact and wake entrainment as well as sink and source terms due to wake 
entrainment and turbulent impact in the group II (see Table 4.1). In the present analysis, some other mechanisms, such as coalescence due to random collision between cap bubbles and breakage due to flow instability, are excluded because only limited data sets taken in a round pipe with the inner diameter of $50.8 \mathrm{~mm}$ are available to validate the two-group model (Hibiki et al., 1998b). In such a relatively small pipe, cap and slug bubbles would rise around the center of the pipe resulting in a minor role of random collision between cap

Table 4.1 Sink and source interactions of bubbles for bubble number density.

\begin{tabular}{|c|c|c|c|c|}
\hline Bubble Group & \multirow{2}{*}{\multicolumn{2}{|c|}{$\begin{array}{l}\text { Coalescence } \\
\text { Random Collision } \\
\text { (Wake Entrainment) }\end{array}$}} & Brea & ap : \\
\hline $\begin{array}{c}\text { Group I } \\
\text { (I+I \& I) }\end{array}$ & & & \multicolumn{2}{|c|}{ Turbulent Impact } \\
\hline & Group I & Group II & Group I & Group II \\
\hline & Sink & - & Source & - \\
\hline \multirow[t]{3}{*}{$\begin{array}{c}\text { Inter-group } \\
(\text { I }+ \text { II \& II) }\end{array}$} & \multicolumn{2}{|c|}{$\begin{array}{l}\text { Wake Entrainment } \\
\text { (Random Collision) }\end{array}$} & \multicolumn{2}{|c|}{$\begin{array}{c}\text { Turbulent Impact } \\
\text { (Surface Instability) } \\
\text { (Shearing-off) }\end{array}$} \\
\hline & Group I & Group II & Group I & Group II \\
\hline & Sink & -1 & Source & -2 \\
\hline \multirow[t]{3}{*}{$\begin{array}{l}\text { Inter-group } \\
\text { (I+I \& II) }\end{array}$} & \multicolumn{2}{|c|}{$\begin{array}{l}\text { (Bubble Cluster Formation) } \\
\text { (Random Collision) } \\
\text { (Wake Entrainment) }\end{array}$} & \multicolumn{2}{|c|}{$\begin{array}{l}\text { (Turbulent Impact) } \\
\text { (Surface Instability) }\end{array}$} \\
\hline & Group I & Group III & Group I & Group II \\
\hline & Sink & Source & Source & Sink \\
\hline \multirow[t]{3}{*}{$\begin{array}{c}\text { Group II } \\
(\text { III+I \& II) }\end{array}$} & \multicolumn{2}{|c|}{$\begin{array}{l}\text { Wake Entrainment } \\
\text { (Random Collision) }\end{array}$} & \multicolumn{2}{|c|}{$\begin{array}{l}\text { Turbulent Impact } \\
\text { (Surface Instability) }\end{array}$} \\
\hline & Group I & Group II & Group I & Group II \\
\hline & - & Sink & - & Source \\
\hline
\end{tabular}

- The mechanisms indicated by bold letters are considered in the present work.

- The mechanisms indicated by brackets should be considered in the future work. $\leq$ Footnotes $>$

*1) This interaction is certainly a source term for the group $I$ in terms of the interfacial area concentration. In this case, although the bubble number density in the group II is not changed by this bubble interaction, the void fraction transport from the group I to II increases total volume of bubbles in the group II, resulting in the increase in the interfacial area concentration in the group II.

*2) This interaction is a sink term for the group II in terms of the interfacial area concentration, because of the void transport from the group II to $I$. 
bubbles. The breakup of cap bubbles would not occur because the maximum cap bubble size approximately given by $40 \sqrt{\sigma / g \Delta \rho}(\approx 10 \mathrm{~cm}$ for air-water flow $)$ (Kocamustafaogullari and Ishii, 1985) is larger than the pipe diameter. Two more mechanisms besides the exclusion of the above two mechanisms are also omitted in this analysis. They are interchange terms due to the complete breakup of a cap bubble (Group II) into small bubbles (Group I) and the coalescence of small bubbles (Group I) into a cap bubble (Group II). As will be shown later, since the ratio in diameter of cap bubbles to small bubbles is about 10 to 20 in the present experimental conditions, these interchanges of bubbles are unlikely to occur. Eventually, six mechanisms listed in Table 4.1 will be considered as sink and source terms in the two-group interfacial area transport equations to be applied at the bubbly-to-slug flow transition in this study.

\subsection{Random Collision Induced Bubble Coalescence}

The coalescence due to random collision of bubbles (Group I) was modeled in the previous paper (Hibiki and Ishii, 1999b; 2000a). The essential part of the model is summarized here. The bubble coalescence is considered to occur due to the bubble random collision induced by turbulence in a liquid phase. For the estimation of bubble-bubble collision frequency, it is assumed that bubbles behave like ideal gas molecules in an isotropic turbulence system. Following the kinetic theory of gases (Loeb, 1927), the frequency of the collision between a single bubble belonging to group I and surrounding bubbles belonging to group I, $f_{\mathrm{RC}, 1}$, can be expressed by assuming the identical spherical bubbles as a function of surface available to the collision and volume available to the collision. Taking account of the excluded volume for bubbles and the overlap of the excluded volume for high void fraction region, the final form of the collision frequency is deduced as:

$$
f_{R C, 1}=\frac{\gamma_{R C, 1} \alpha_{1} \varepsilon^{1 / 3}}{D_{b, 1}^{2 / 3}\left(\alpha_{R C, \text { max }}-\alpha\right)}
$$

where $\gamma_{R C, 1}, \varepsilon$, and $D_{b, \mathrm{k}}$ define an adjustable parameter, the energy dissipation, and the diameter of the bubble belonging to the $k$-group, respectively. $\alpha_{\mathrm{k}}$ and $\alpha$ are the void fraction of bubbles in the $k$-group, and the total void fraction of all bubbles in a finite volume element of the mixture, respectively. The maximum allowable void fraction, $\alpha_{\mathrm{RC}, \max }$, was set at 0.741 , which corresponds to the void fraction for close-packed bubbles 
(Moore, 1972). A short discussion on an order of magnitude of the collision frequency is given in Appendix A.

In order to obtain the bubble coalescence rate, it is necessary to determine a coalescence efficiency, $\lambda_{\mathrm{RC}, 1}$. The coalescence efficiency can be derived from the liquid film thinning model as (Oolman and Blanch, 1986a; 1986b; Prince and Blanch, 1990):

$$
\lambda_{R C, 1}=\exp \left(-K_{R C, 1} \sqrt[6]{\frac{D_{b, 1}^{5} \rho_{f}^{3} \varepsilon^{2}}{\sigma^{3}}}\right),
$$

where $K_{\mathrm{RC}, 1}, \rho_{\mathrm{f}}$ and $\sigma$ are an experimental coefficient, the liquid density and the surface tension, respectively.

Finally, the bubble coalescence rate, $S_{\mathrm{RC}, 1}$, and the decrease rate of the interfacial area concentration, $\Phi_{\mathrm{RC}, 1}$, are expressed as:

$$
\begin{aligned}
& S_{R C, 1}=-f_{R C, 1} \cdot n_{b, 1} \cdot \lambda_{R C, 1}=-\frac{\gamma_{R C, 1}^{\prime} \alpha_{1}^{2} \varepsilon^{1 / 3}}{D_{b, 1}^{1 / 3}\left(\alpha_{R C, \max }-\alpha\right)} \exp \left(-K_{R C, 1} \sqrt{\frac{D_{b, 1}^{5} \rho_{f}^{3} \varepsilon^{2}}{\sigma^{3}}}\right), \\
& \Phi_{R C, 1}=-\frac{1}{3 \psi_{1}}\left(\frac{\alpha_{1}}{a_{i, 1}}\right)^{2} \cdot f_{R C, 1} \cdot n_{b, 1} \cdot \lambda_{R C, 1}=-\left(\frac{\alpha_{1}}{a_{i, 1}}\right)^{2} \frac{\Gamma_{R C, 1} \alpha_{1}^{2} \varepsilon^{1 / 3}}{D_{b, 1}^{1 / 3}\left(\alpha_{R C, \max }-\alpha\right)} \exp \left(-K_{R C, 1} \sqrt{\frac{D_{b, 1}^{5} \rho_{f}^{3} \varepsilon^{2}}{\sigma^{3}}}\right),
\end{aligned}
$$

where $n_{\mathrm{b}, \mathrm{k}}$ denotes the number density of bubbles belonging to the $k$-group given by Eq.(4.5). $\gamma^{\prime}{ }_{\mathrm{RC}, 1}$ and $\Gamma_{\mathrm{RC}, 1}$ are an adjustable parameter, and an experimental coefficient, respectively.

$$
n_{b, k}=\frac{6 \alpha_{k}}{\pi D_{b, k}^{3}} \text {. }
$$

\subsection{Wake-entrainment Induced Bubble Coalescence}

The model of wake-entrainment induced bubble coalescence was proposed for bubbles with uniform size distribution (Wu et al., 1998b). Here, the model is modified for the collision between spherical/distorted bubble and cap/slug bubble. In general, when bubbles enter the wake region of a leading cap bubble, they will accelerate and may collide with the leading one (Otake et al., 1977; Bilicki and Kestin, 1987; Tsuchiya et al., 1989; Stewart, 1995). According to Otake et al. (1977), there was the critical distance, $L_{\mathrm{WE}}$, at which the leading bubble began to exert noticeable influence on the following one. In their experiment, the critical distance was found to be about 3-to 4-fold diameter of the leading 
bubble. In the present model, all bubbles in the wake region are assumed to collide with the leading cap bubble which is approximated to be large spherical bubble with the equivalent volume of the cap bubble to obtain the analytical form of the velocity distribution in the wake region (see Fig.4.2). The distortion due to this assumption will be adjusted by a tuning parameter in the final equation. The number of small bubbles (Group I), $N_{\mathrm{wE}}$, inside the effective wake region, $V_{\mathrm{WE}}$ is given by:

$N_{W E}=V_{W E} \cdot n_{b, 1}=\frac{\pi}{4} D_{b, 2}^{2}\left(L_{W E}-\frac{D_{b, 2}}{2}\right) \cdot\left(\frac{6 a_{1}}{\pi D_{b, 1}^{3}}\right)$.

The collision rate per unit mixture volume, $f_{\mathrm{WE}, 12}$, can be obtained by assuming that all bubbles in the wake region collide with the leading cap bubble within the average time interval, $\Delta T$ as:

$f_{W E, 12}=\gamma_{W E, 12} \frac{N_{W E}}{\Delta T} \cong \frac{\gamma_{W E, 12}^{\prime} D_{b, 2}^{2} \alpha_{1} \overline{v_{W E}}}{D_{b, 1}^{3}}$

where $\gamma_{W E, 12}$ and $\gamma$ 'WE,12 are adjustable parameters. $\overline{v_{W E}}$ is the average relative velocity between the leading cap bubble and the small bubble in the wake region, respectively. Schlichting (1979) gave the analytical expression of the non-dimensionalized relative velocity as:

$\frac{v_{W E}}{U_{\infty}} \approx\left(\frac{C_{D} A}{\beta^{2} y^{2}}\right)^{1 / 3}$,

where $A, \beta$, and $y$ are the frontal area of the bubble, the ratio of the mixing length and the width of the wake, and the distance measured from the center of the leading bubble, respectively. The drag coefficient, $C_{D}$, can be approximated to be $8 / 3$ for $E o\left(\equiv g \Delta \rho D_{b}{ }^{2} / \sigma\right)>40$ (Ishii and Chawla, 1979; Tomiyama et al., 1995). It is clear that in thismodel $U_{\infty}$ represents the relative velocity between the cap bubble and the liquid phase (Bilicki and Kestin, 1987),

$U_{\infty}=v_{z, 2}-v_{f}$

where $v_{\mathrm{z}, 2}$ and $v_{\mathrm{f}}$ define the cap bubble velocity and liquid velocity as defined by Eq.(3.23) and Eq.(3.34), respectively. 


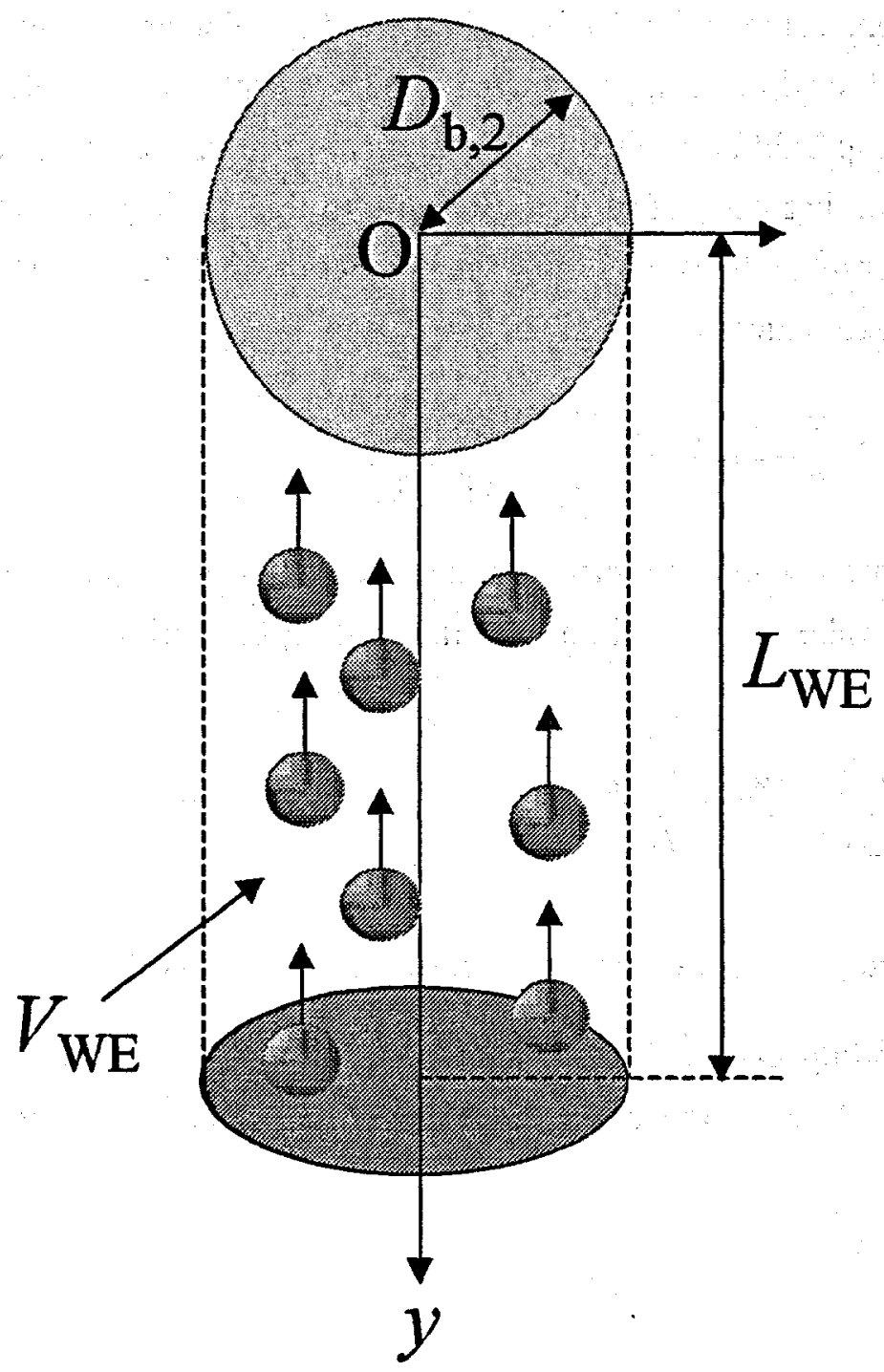

Figure 4.2. Schematic diagram of wake entrainment model.

$$
v_{f}=\frac{j_{f}}{1-\alpha},
$$

where $j_{\mathrm{f}}$ is the superficial liquid velocity. The average relative velocity in the wake region can be obtained by integrating Eq.(3.32) over the effective wake length as:

$$
\overline{v_{W E}}=\frac{\gamma_{W E, 12}^{\prime \prime}\left(v_{z, 2}-v_{f}\right)}{L_{W} / D_{b, 2}-1 / 2}\left\{\left(\frac{L_{W}}{D_{b, 2} / 2}\right)^{1 / 3}-1\right\} \text {. }
$$

where $\gamma$ "WE,12 is an adjustable parameter. The bubble collision rate per unit mixture 
volume due to the wake entrainment mechanism is given by the following equation, providing that $L_{\mathrm{W}} / D_{\mathrm{b}, 2}$ is treated as a constant depending on the fluid properties (Wu et al., 1998b).

$f_{W E, 12}=\frac{\gamma_{W E, 12}^{m} D_{b, 2}^{2} \alpha_{1}}{D_{b, 1}^{3}}\left(v_{z, 2}-v_{f}\right)$.

where $\gamma$ " "WE,12 is an adjustable parameter. The coalescence efficiency of two bubbles with different sizes, $\lambda_{\mathrm{WE}, 12}$, would be expressed by using the equivalent diameter, $D_{\text {eq.H-C, }}$ given by Chesters and Hoffman (1982) (see Eq.(4.13)) as Eq.(4.14).

$$
\begin{aligned}
& \frac{1}{D_{\text {aq,H-C }}}=\frac{1}{2}\left(\frac{1}{D_{b, 1}}+\frac{1}{D_{b, 2}}\right), \\
& \lambda_{W E, 12}=\exp \left\{-K_{W E, 12} \sqrt{\frac{\rho_{f}^{3} \varepsilon^{2}}{\sigma^{3}}\left(\frac{D_{b, 1} D_{b, 2}}{D_{b, 1}+D_{b, 2}}\right)^{5}}\right\},
\end{aligned}
$$

where $K_{\mathrm{WE}, 12}$ is an experimental coefficient, which is related to $K_{\mathrm{RC}, 1}$ as $2^{5 / 6} \times K_{\mathrm{RC}, 1}\left(=1.78 K_{\mathrm{RC}, 1}\right)$.

Finally, the bubble coalescence rate, $S_{\mathrm{WE}, 12}$, and the decrease rate of the interfacial area concentration, $\Phi_{\mathrm{WE}, 12}$, are expressed as:

$$
\begin{aligned}
& S_{W E, 12}=-f_{W E, 12} \cdot n_{b, 2} \cdot \lambda_{W E, 12}=-\frac{\gamma_{W E, 12}^{m n} \alpha_{1} \alpha_{2}}{D_{b, 1}^{3} D_{b, 2}}\left(v_{z, 2}-v_{f}\right) \exp \left\{-K_{W E, 12} \sqrt{\frac{\rho_{f}^{3} \varepsilon^{2}}{\sigma^{3}}\left(\frac{D_{b, 1} D_{b, 2}}{D_{b, 1}+D_{b, 2}}\right)^{5}}\right\} \\
& \Phi_{W E, 12}=-\frac{1}{3 \psi_{1}}\left(\frac{\alpha_{1}}{a_{i, 1}}\right)^{2} \cdot f_{W E, 12} \cdot n_{b, 2} \cdot \lambda_{W E, 12} \\
& =-\left(\frac{\alpha_{1}}{a_{i, 1}}\right)^{2} \frac{\Gamma_{W E, 12} \alpha_{1} \alpha_{2}}{D_{b, 1}^{3} D_{b, 2}}\left(v_{s, 2}-v_{f}\right) \exp \left\{-K_{W E, 12} \sqrt{\frac{\rho_{f}^{3} \varepsilon^{2}}{\sigma^{3}}\left(\frac{D_{b, 1} D_{b, 2}}{D_{b, 1}+D_{b, 2}}\right)^{5}}\right\}
\end{aligned}
$$

where $\gamma$ " "WE,12 and $\Gamma_{\mathrm{WE}, 12}$ are adjustable parameters.

In a similar way, the frequency of the collision between cap bubbles (Group II) due to wake entrainment, $f_{\mathrm{WE}, 2}$, can be derived by replacing $D_{\mathrm{b}, 1}$ with $D_{\mathrm{b}, 2}$ and $\alpha_{1}$ with $\alpha_{2}$ in Eq.(4.7) as: 
$f_{W E, 2}=\frac{\gamma_{W E, 2} \alpha_{2} \overline{v_{W E}}}{D_{b, 2}}$

where $\gamma_{\mathrm{WE}, 2}$ is an adjustable parameter. The coalescence efficiency, $\lambda_{\mathrm{WE}, 2}$, is given by:

$\lambda_{W E, 2}=\exp \left(-K_{W E, 2} \sqrt[6]{\frac{D_{b, 2}^{5} \rho_{f}^{3} \varepsilon^{2}}{\sigma^{3}}}\right)$,

where $K_{\mathrm{WE}, 2}$ is an experimental constant, which is equal to $K_{\mathrm{RC}, 1}$.

Finally, the bubble coalescence rate, $S_{\mathrm{WE}, 2}$, and the decrease rate of the interfacial area concentration, $\Phi_{\mathrm{WE}, 2 \text {, are expressed as: }}$

$$
\begin{aligned}
& S_{W E, 2}=-f_{W E, 2} \cdot n_{b, 2} \cdot \lambda_{W E, 2}=-\frac{\gamma_{W E, 2}^{\prime} \alpha_{2}^{2}}{D_{b, 2}^{4}}\left(v_{z, 2}-v_{f}\right) \exp \left\{-K_{W E, 2} \sqrt{\frac{D_{b, 2}^{5} \rho_{f}^{3} \varepsilon^{2}}{\sigma^{3}}}\right\}, \\
& \Phi_{W E, 2}=-\frac{1}{3 \psi_{2}}\left(\frac{\alpha_{2}}{a_{i, 2}}\right)^{2} \cdot f_{W E, 2} \cdot n_{b, 2} \cdot \lambda_{W E, 2} \\
& =-\left(\frac{\alpha_{2}}{a_{i, 2}}\right)^{2} \frac{\Gamma_{W E, 2} \alpha_{2}^{2}}{D_{b, 2}^{4}}\left(v_{z, 2}-v_{f}\right) \exp \left\{-K_{W E, 2} \sqrt[6]{\frac{D_{b, 2}^{5} \rho_{f}^{3} \varepsilon^{2}}{\sigma^{3}}}\right\} .
\end{aligned}
$$

where $\gamma^{\prime}{ }^{\mathrm{WE}, 2}$ and $\Gamma_{\mathrm{WE}, 2}$ are adjustable parameters.

\subsection{Bubble Breakup Due to Turbulent Impact}

The breakup of bubbles (Group I) due to turbulent impact was modeled in the previous paper (Hibiki and Ishii, 1999b; 2000a). The essential part of the model is summarized here. The bubble breakup is considered to occur due to the collision of the turbulent eddy with the bubble. For the estimation of bubble-eddy collision frequency, it is assumed that the eddies and bubbles behave like ideal gas molecules. Furthermore, the following assumptions are made for the modeling of the bubble-eddy collision rate: (i) the turbulence is isotropic; (ii) the eddy size, $D_{\mathfrak{e}}$, of interest lies in the inertial subrange; (iii) the eddy with the size from c $D_{\mathrm{b}}$ to $D_{\mathrm{b}}$ can break up the bubble with the size of $D_{\mathrm{b}}$, since larger eddies have the tendency to transport the bubble rather than to break it and smaller eddies do not have enough energy to break it. Following the kinetic theory of gases (Loeb, 1927), the frequency of the collision between a single turbulent eddy and surrounding bubbles belonging to group $I, f_{\mathrm{T}, 1}$, can be expressed by assuming the identical spherical bubbles and spherical eddies as a function of the surface available to the collision and the volume 
available to the collision. Taking account of the excluded volume for bubbles and eddies, and the overlap of the excluded volume for high void fraction region, the final form of the collision frequency is deduced as:

$$
f_{T I, 1}=\frac{\gamma_{T, 1} \alpha_{1} \varepsilon^{1 / 3}}{D_{b, 1}^{2 / 3}\left(\alpha_{T I, \max }-\alpha\right)}
$$

where $\gamma_{\mathrm{TL}, 1}$ is an adjustable parameter. Although this parameter is basically a function of $c\left(=D_{\mathrm{d}} / D_{\mathrm{b}}\right)$ and the degree of the overlap of the excluded volume, it is treated as a constant for simplicity. The model with this assumption still worked practically (Hibiki and Ishii, 1999b; 2000a). The maximum allowable void fraction, $\alpha_{\mathrm{Tl}, \max }(\approx 0.741)$ in Eq.(4.21) is approximately the same as that in Eq.(4.1), if eddies with almost the same size of bubbles is assumed to break up the bubbles. Consequently, the functional form of the frequency of the bubble-eddy random collision, Eq.(4.21), looks similar to that of the frequency of the bubble-bubble random collision, Eq.(4.1).

In order to obtain the bubble breakup rate, it is necessary to determine a breakup efficiency, $\lambda_{\mathrm{TL}, 1}$. The breakup efficiency can be derived from the relationship between the energy of a single eddy and the energy required for breakup as (Tsouris and Tavlarides, 1994; Hibiki and Ishii, 1999b; 2000a):

$\lambda_{T T, 1}=\exp \left(-\frac{K_{T R, 1} \sigma}{\rho_{f} D_{b, 1}^{5 / 3} \varepsilon^{2 / 3}}\right)$,

where $K_{\mathrm{T}, 1}$ is an experimental coefficient.

Finally, the bubble breakup rate, $S_{\mathrm{Th}, 1}$, and the decrease rate of the interfacial area concentration, $\Phi_{\mathrm{TL}, 1}$, are expressed as:

$$
\begin{aligned}
& S_{T I, 1}=f_{I I, 1} \cdot n_{e, 1} \cdot \lambda_{T I, 1}=\frac{\gamma_{I, 1}^{\prime} \alpha_{1}(1-\alpha) \varepsilon^{1 / 3}}{D_{b, 1}^{11 / 3}\left(\alpha_{T I, \max }-\alpha\right)} \exp \left(-\frac{K_{T I, 1} \sigma}{\rho_{f} D_{b, 1}^{S / 3} \varepsilon^{2 / 3}}\right), \\
& \Phi_{\pi, 1}=\frac{1}{3 \psi_{1}}\left(\frac{\alpha_{1}}{a_{i, 1}}\right)^{2} \cdot f_{T I, 1} \cdot n_{e, 1} \cdot \lambda_{T I, 1}=\left(\frac{\alpha_{1}}{a_{i, 1}}\right)^{2} \frac{\Gamma_{T, 1} \alpha_{1}(1-\alpha) \varepsilon^{1 / 3}}{D_{b, 1}^{11 / 3}\left(\alpha_{I I, \max }-\alpha\right)} \exp \left(-\frac{K_{T I, 1} \sigma}{\rho_{f} D_{b, 1}^{5 / 3} \varepsilon^{2 / 3}}\right)
\end{aligned}
$$

where $n_{\mathrm{c}, \mathrm{k}}$ denotes the number of eddies with size from $c \cdot D_{\mathrm{b}, \mathrm{k}}$ to $D_{\mathrm{b}, \mathrm{k}}$ per volume of two-phase mixture given by Eq.(4.25) (Azbel and Athanasios, 1983; Hibiki and Ishii, $1999 \mathrm{~b} ; 2000 \mathrm{a})$. $\gamma_{\pi, 1}$ and $\Gamma_{\mathrm{T}, 1}$ are adjustable parameters. 
$n_{e, k}=\frac{0.267}{D_{b, k}^{3}}\left(\frac{1}{c^{3}}-1\right)(1-\alpha)$.

In a similar way, the frequency of the collision between the turbulent eddy and the bubble belonging to the group II, $f_{\mathrm{TL}, 2}$, can be derived as:

$f_{\pi 1,2}=\frac{\gamma_{\pi, 2} \alpha_{2} \varepsilon^{1 / 3}}{D_{b, 2}^{2 / 3}\left(\alpha_{\pi I, \max }-\alpha\right)}$

where $\gamma_{\pi, 2}$ is an adjustable parameter Here, the distribution function, $\zeta$, is defined to specify the rate of the collision between turbulent eddies and cap bubbles (Group II), producing small daughter bubbles (Group I) to the total collision. Thus, $\zeta f_{T, 2}$ and (1-ऽ) $f_{\mathrm{TI}, 2}$ contribute to the breakup of a cap bubble (Group II) into cap and small bubbles (Group I and II) and the breakup of a cap bubble (Group II) into cap bubbles (Group II), respectively. The breakup efficiencies for each breakup can be derived from the relationship between the energy of a single eddy and the energy required for breakup (Tsouris and Tavlarides, 1994; Hibiki and Ishii, 1999b; 2000a). The efficiencies of the breakup of a cap bubble into a smaller cap bubble (Group II) and a small bubble (Group I) or a cap bubble into two identical cap bubbles (Group II) are expressed by Eqs.(4.27) and (4.28), respectively.

$\lambda_{\pi, 12}=\exp \left(-\frac{\left.K_{T, 12} \sigma\left(D_{b, 2}^{3}-D_{b, 1}^{3}\right)^{2 / 3}+\left(D_{b, 1}^{2}-D_{b, 2}^{2}\right)\right\}}{\rho_{f} D_{b, 2}^{1 / 3} \varepsilon^{2 / 3}}\right)$,

where $K_{\mathrm{TL}, 12}$ is an experimental coefficient, which is related to $K_{\mathrm{TL}, 1}$ as $\left\{1 /\left(2^{1 / 3}-1\right)\right\} \times K_{\mathrm{TH}, 1}$ $\left(=3.85 K_{\mathrm{T}, 1}\right)$.

$\lambda_{T I, 2}=\exp \left(-\frac{K_{T I, 2} \sigma}{\rho_{f} D_{b, 2}^{5 / 3} \varepsilon^{2 / 3}}\right)$,

where $K_{\mathrm{T}, 2}$ is an experimental constant, which is equal to $K_{\mathrm{T}, 1}$.

Finally, the bubble breakup rates, $S_{\mathrm{TT}, 12}$ and $S_{\mathrm{TT}, 2}$, and the decrease rates of the interfacial area concentration, $\Phi_{\mathrm{TH}, 12}$ and $\Phi_{\mathrm{TL}, 2}$, are expressed as:

$$
\begin{aligned}
& S_{T I, 12}=\varsigma \cdot f_{T l, 2} \cdot n_{e, 2} \cdot \lambda_{T I, 12} \\
& =\frac{\gamma_{\pi, 11} \alpha_{2}(1-\alpha) \varepsilon^{1 / 3}}{D_{b, 2}^{11 / 3}\left(\alpha_{T 1, \max }-\alpha\right)} \exp \left(-\frac{\left.K_{T 7,12} \sigma\left(D_{b, 2}^{3}-D_{b, 1}^{3}\right)^{2 / 3}+\left(D_{b, 1}^{2}-D_{b, 2}^{2}\right)\right)}{\rho_{f} D_{b, 2}^{1 / 3} \varepsilon^{2 / 3}}\right),
\end{aligned}
$$




$$
\begin{aligned}
& \Phi_{T I, 12}=\frac{1}{3 \psi_{1}}\left(\frac{\alpha_{1}}{a_{i, 1}}\right)^{2} \cdot \varsigma \cdot f_{T I, 2} \cdot n_{e, 2} \cdot \lambda_{T I, 12} \\
& =\left(\frac{\alpha_{1}}{a_{i, 1}}\right)^{2} \frac{\Gamma_{T I, 12} \alpha_{2}(1-\alpha) \varepsilon^{1 / 3}}{D_{b, 2}^{11 / 3}\left(\alpha_{T I, \max }-\alpha\right)} \exp \left(-\frac{\left.K_{T I, 22} \sigma\left(D_{b, 2}^{3}-D_{b, 1}^{3}\right)^{2 / 3}+\left(D_{b, 1}^{2}-D_{b, 2}^{2}\right)\right\}}{\rho_{f} D_{b, 2}^{11 / 3} \varepsilon^{2 / 3}}\right) \\
& S_{T I, 2}=(1-\varsigma) \cdot f_{T I, 2} \cdot n_{e, 2} \cdot \lambda_{T I, 2}=\frac{\gamma_{\pi, 2}^{\prime} \alpha_{2}(1-\alpha) \varepsilon^{1 / 3}}{D_{b, 2}^{11 / 3}\left(\alpha_{T 1, \max }-\alpha\right)} \exp \left(-\frac{K_{T I, 2} \sigma}{\rho_{f} D_{b, 2}^{5 / 3} \varepsilon^{2 / 3}}\right) \\
& \Phi_{T I, 2}=\frac{1}{3 \psi_{2}}\left(\frac{\alpha_{2}}{a_{i, 2}}\right)^{2} \cdot(1-\varsigma) \cdot f_{T I, 2} \cdot n_{e, 2} \cdot \lambda_{T I, 2} \\
& =\left(\frac{\alpha_{2}}{a_{i, 2}}\right)^{2} \frac{\Gamma_{T I, 2} \alpha_{2}(1-\alpha) \varepsilon^{1 / 3}}{D_{b, 2}^{11 / 3}\left(\alpha_{T, \max }-\alpha\right)} \exp \left(-\frac{K_{T I, 2} \sigma}{\rho_{f} D_{b, 2}^{5 / 3} \varepsilon^{2 / 3}}\right)
\end{aligned}
$$

where $\Gamma_{\mathrm{T}, 12}$ and $\Gamma_{\mathrm{Tl}, 2}$ are adjustable parameters. Although these parameters are basically a function of $c\left(=D_{d} / D_{\mathrm{b}}\right)$, the distribution function and the degree of the overlap of the excluded volume, they are treated as constants for simplicity.

\subsection{Final Equations of Source and Sink Terms in Two-group}

\section{Interfacial Area Transport Equations}

As will be discussed in the next section, the derived sink and source terms will be evaluated by area-averaged flow parameters, which were measured in a vertical pipe under adiabatic and steady conditions. Under adiabatic and steady conditions, the one-dimensional form of two-group interfacial area transport equations and the void fraction transport equation without considering the bubble size distribution are given by

$$
\begin{aligned}
& \frac{d}{d z}\left(\left\langle a_{i, 1}\right\rangle\left\langle\left\langle v_{i z, 1}\right\rangle\right\rangle_{a}\right)=\left\langle\Phi_{R C, 1}\right\rangle+\left\langle\Phi_{T I, 1}\right\rangle+\left\langle\Phi_{W E, 12}\right\rangle+\left\langle\Phi_{T I, 12}\right\rangle+\left\langle\Phi_{V T, 1}\right\rangle, \\
& \frac{d}{d z}\left(\left\langle a_{i, 2}\right\rangle\left\langle\left\langle v_{i z, 2}\right\rangle\right\rangle_{a}\right)=\left\langle\Phi_{W E, 2}\right\rangle+\left\langle\Phi_{T I, 2}\right\rangle+\left\langle\Phi_{V T, 2}\right\rangle \\
& \frac{d}{d z}\left(\left\langle\alpha_{1}\right\rangle\left\langle\left\langle v_{z, 1}\right\rangle\right\rangle\right)=\left\langle\Psi_{W E, 12}\right\rangle+\left\langle\Psi_{T I, 12}\right\rangle+\left\langle\Psi_{V T, 1}\right\rangle
\end{aligned}
$$

The sink and source terms in the above equations are listed in Tables 4.2 and 4.3. Table 4.4 also shows the list of unknown quantities to be calculated and measured quantities. In these equations, the value of the energy dissipation, $\langle\varepsilon\rangle$, is assumed to be proportional to the energy dissipation, $\left\langle\varepsilon_{0}\right\rangle$, which is simply obtained from the mechanical energy 
58

equation as (Kocamustafaogullari et al., 1994):

$$
\langle\varepsilon\rangle=\kappa\left\langle\varepsilon_{0}\right\rangle, \quad\left\langle\varepsilon_{0}\right\rangle=\frac{\langle j\rangle}{\rho_{m}}\left(-\frac{d P}{d z}\right)_{F},
$$

where $j, \rho_{\mathrm{m}}, \kappa$, and $(-d P / d z)_{\mathrm{F}}$ refer to the mixture volumetric flux, the mixture density, the coefficient, and the pressure loss per unit length due to friction, respectively.

Table 4.2 Summary of the equations to predict the interfacial area concentration.

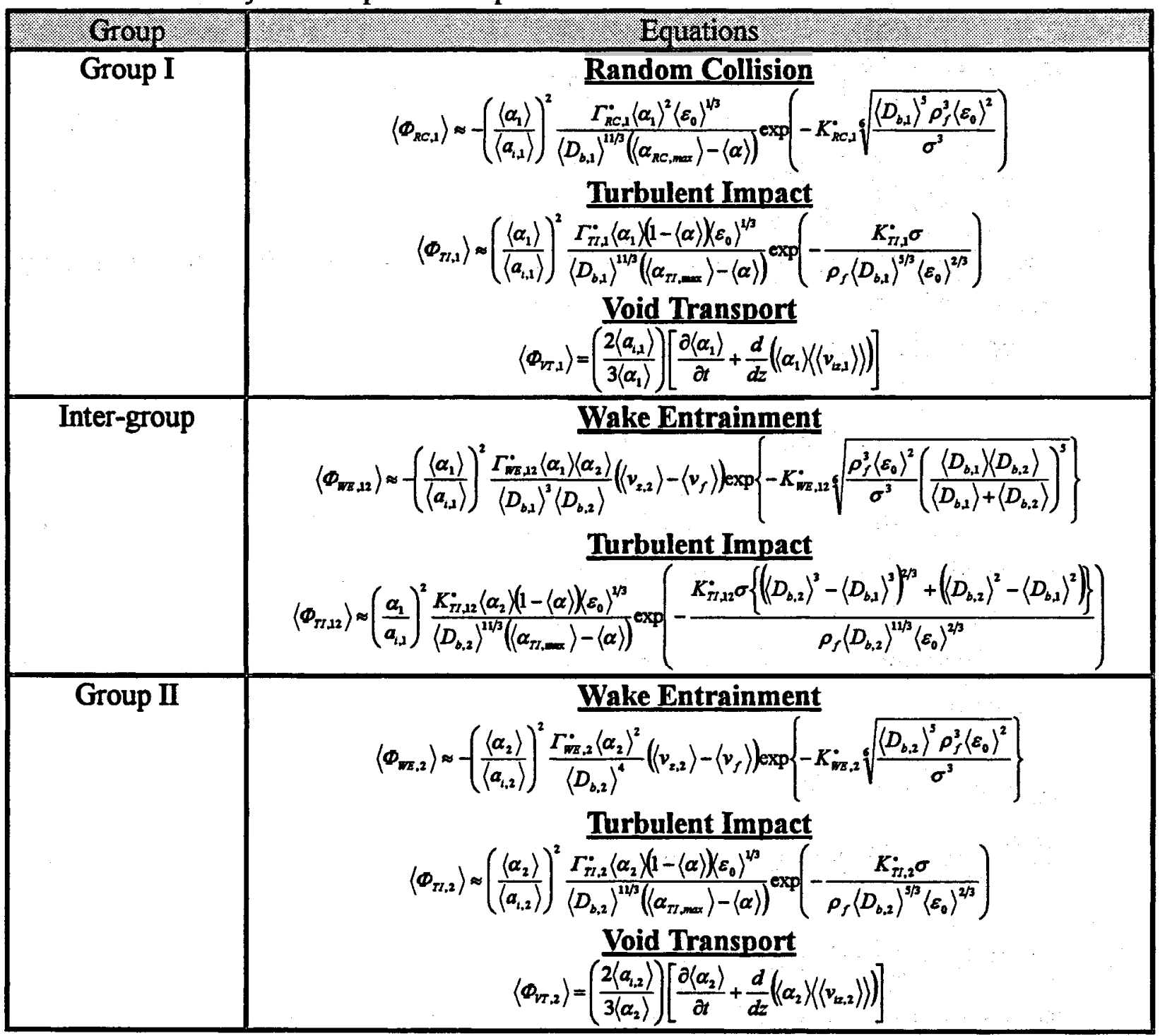


Table 4.3 Summary of the equations to predict the void fraction.

\begin{tabular}{|c|c|}
\hline Mechanisms & Equations \\
\hline Wake Entrainment & 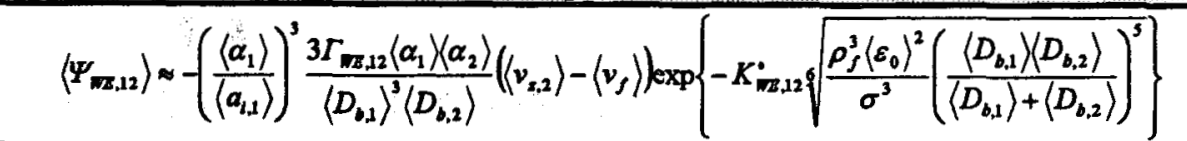 \\
\hline Turbulent Impact & 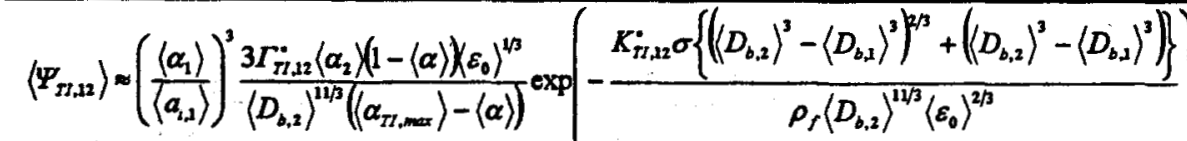 \\
\hline Pressure Variation & $\left\langle\Psi_{r_{r 1}}\right\rangle=-\frac{\left\langle\alpha_{1}\right\rangle\left\langle\left\langle v_{r_{1}}\right\rangle\right\rangle}{\rho_{g}} \frac{d \rho_{g}}{d z}$ \\
\hline $\begin{array}{l}\text { Balance of Void } \\
\text { Fraction }\end{array}$ & $\langle\alpha\rangle=\left\langle\alpha_{1}\right\rangle+\left\langle\alpha_{2}\right\rangle$ \\
\hline
\end{tabular}

Table 4.4 List of measured quantities and unknown quantities to be calculated

\begin{tabular}{|c|c|c|}
\hline $\begin{array}{c}\text { Measured or Calculated } \\
\text { Quantities }\end{array}$ & Quantities & Remarks \\
\hline $\begin{array}{l}\text { Unknown Quantities } \\
\text { to be calculated }\end{array}$ & $\begin{array}{c}\text { Void Fraction, }\left\langle\alpha_{1}\right\rangle \\
\text { Void Fraction, }\left\langle\alpha_{2}\right\rangle \\
\text { IAC, }\left\langle a_{\mathrm{i}, 1}\right\rangle \\
\text { IAC, }\left\langle a_{\mathrm{i}, 2}\right\rangle \\
\text { Bubble Diameter, }\left\langle D_{\mathrm{b}, 1}\right\rangle \\
\text { Bubble Diameter, }\left\langle D_{\mathrm{b}, 2}\right\rangle \\
\text { Gas Velocity, }\left\langle v_{\mathrm{z}, 1}\right\rangle \\
\text { Gas Velocity, }\left\langle v_{\mathrm{z}, 2}\right\rangle\end{array}$ & $\begin{array}{c}\text { Calculated from Void Transport Eq. } \\
\left.\text { Calculated from }<\alpha>-<\alpha_{1}\right\rangle \\
\text { Calculated from IAC Transport Eq. } \\
\text { Calculated from IAC Transport Eq. } \\
\text { Calculated from } 6<\alpha_{1}>\left|<a_{\mathrm{i}, 1}\right\rangle \\
\text { Calculated from } 6<\alpha_{2}>\left|<a_{\mathrm{i}, 2}\right\rangle \\
\left.\text { Assumption: }\left\langle v_{\mathrm{z}}\right\rangle=<<v_{\mathrm{z}, 1}\right\rangle \\
\text { Assumption: }\left\langle v_{z}\right\rangle=\left\langle v_{\mathrm{z}, 2}\right\rangle\end{array}$ \\
\hline Measured Quantities & $\begin{array}{c}\text { Void Fraction, }\langle\alpha\rangle \\
\text { Gas Velocity, }\left\langle v_{z}\right\rangle \\
\text { Liquid Velocity }\left\langle v_{\mathbf{f}}\right\rangle\end{array}$ & $\begin{array}{c}\text { Measured } \\
\text { Calculated from }\left\langle j_{\mathrm{B}}><<>\right. \\
\text { Calculated from }<j_{\mathrm{P}}>/(1-<>)\end{array}$ \\
\hline Initial Values & $\left\langle\alpha_{1}\right\rangle,\left\langle\alpha_{2}\right\rangle,\left\langle a_{i, 1}\right\rangle,\left\langle a_{i, 2}\right\rangle$ & Measured values at $z / D=6.00$ \\
\hline
\end{tabular}




\section{RESULTS AND DISCUSSION}

\subsection{Data Base Used for Evaluation of the Derived Model}

In order to evaluate the derived models of the sink and source terms in the interfacial area transport equation, the authors applied the double sensor probe method to measure local flow parameters of adiabatic air-water bubbly flows in vertical round pipes at the Thermal-hydraulics and Reactor Safety Laboratory in Purdue University (Hibiki and Ishii, 1997; 1999a; Hibiki et al., 1998a; 1998b). The dimensions of the flow loop used in this study are tabulated in Table 5.1. The flow conditions in the experiment are shown in Table 5.2 and Fig.5.1. In Fig.5.1, the solid and broken lines indicate the flow regime transition boundaries predicted by the model of Taitel et al. (1980) and the phase distribution pattern transition boundaries given by Serizawa and Kataoka (1988), which were determined based on experiments performed by different researchers with different types of bubble injections in round pipes ( $20 \mathrm{~mm} \leq D \leq 86.4 \mathrm{~mm}$ ). In the flow conditions surrounded by the dotted line, cap bubbles were observed. The superficial gas velocities were roughly determined so as to provide the same void fractions among different conditions of superficial liquid velocity, namely $\left\langle\alpha_{z / D}=53.5\right\rangle=5,10,20$, and $25 \%$. For $\left\langle j_{\mathrm{p}}\right\rangle=5.00 \mathrm{~m} / \mathrm{s}$, two additional data sets at $\left\langle\alpha_{z / D=53.5}\right\rangle=35$ and $45 \%$ were also acquired to verify the applicability of the derived two-group model to the finely dispersed bubbly flow region. Thus, the flow conditions covered most of a bubbly flow region, including the bubbly-to-slug flow transition region and the finely dispersed bubbly flow region. As

Table 5.1 Dimensions of flow loop used in the experiment (Hibiki et al., 1998b).

\begin{tabular}{|c|c|}
\hline Pipe Inner Diameter, $D$ & $50.8 \mathrm{~mm}$ \\
\hline Pipe Length, $L$ & $3061 \mathrm{~mm}$ \\
\hline Axial Measuring Station, $2 / D$ & $6.00,30.3,53.5$ \\
\hline Air Injection Method & Porous Pipe $(40 \mu \mathrm{m})$ \\
\hline Pump Power (Maximum Superficial & $\sim 10 \mathrm{~m} / \mathrm{s}$ \\
Liquid Velocity) & \\
\hline Air Supply System & $9.91 \mathrm{~m}^{3}$ air tank pressurized to $0.929 \mathrm{MPa}$ \\
\hline
\end{tabular}


Table 5.2. Flow conditions in the experiment using a $50.8 \mathrm{~mm}$-diameter pipe (Hibiki et al., 1998b).

\begin{tabular}{|c|c|c|c|c|c|c|}
\hline $\begin{array}{c}\langle j \mathrm{j}\rangle \\
{[\mathrm{m} / \mathrm{s}]}\end{array}$ & $\begin{array}{c}\left\langle j_{\mathrm{g}, 0}\right\rangle \\
{[\mathrm{m} / \mathrm{s}]}\end{array}$ & $\begin{array}{c}\left\langle j_{\mathrm{g}, 0}\right\rangle \\
{[\mathrm{m} / \mathrm{s}]}\end{array}$ & $\begin{array}{c}\left\langle j_{\mathrm{g}, 0}\right\rangle \\
{[\mathrm{m} / \mathrm{s}]}\end{array}$ & $\begin{array}{c}\left\langle j_{\mathrm{g}, 0}\right\rangle \\
{[\mathrm{m} / \mathrm{s}]}\end{array}$ & $\begin{array}{c}\left\langle j_{\mathrm{g}, 0}\right\rangle \\
{[\mathrm{m} / \mathrm{s}]}\end{array}$ & $\begin{array}{c}\left\langle j_{\mathrm{g}, 0}\right\rangle \\
{[\mathrm{m} / \mathrm{s}]}\end{array}$ \\
\hline 0.491 & 0.0275 & 0.0556 & 0.129 & 0.190 & N/A & N/A \\
$\left(\left\langle\alpha_{z / D=53.5}\right\rangle[\%]\right)$ & $(4.90)$ & $(9.20)$ & $(19.2)$ & $(25.9)$ & & \\
\hline 0.986 & 0.0473 & 0.113 & 0.242 & 0.321 & N/A & N/A \\
$\left(<\alpha_{z / D=53.5}>[\%]\right)$ & $(5.12)$ & $(10.8)$ & $(20.3)$ & $(23.1)$ & & \\
\hline 2.01 & 0.103 & 0.226 & 0.471 & 0.624 & N/A & N/A \\
$\left(\left\langle\alpha_{z / D=53.5}>[\%]\right)\right.$ & $(5.68)$ & $(10.8)$ & $(18.3)$ & $(22.8)$ & & \\
\hline 5.00 & 0.245 & 0.518 & 1.11 & 1.79 & 2.87 & 3.90 \\
$\left(\left\langle\alpha_{z / D=53.5}>[\%]\right)\right.$ & $(5.41)$ & $(10.6)$ & $(20.0)$ & $(28.1)$ & $(36.6)$ & $(44.2)$ \\
\hline
\end{tabular}

Note) Cap bubbles were observed in flow conditions shown in gray-colored columns. $\mathrm{N} / \mathrm{A}=$ Not available.

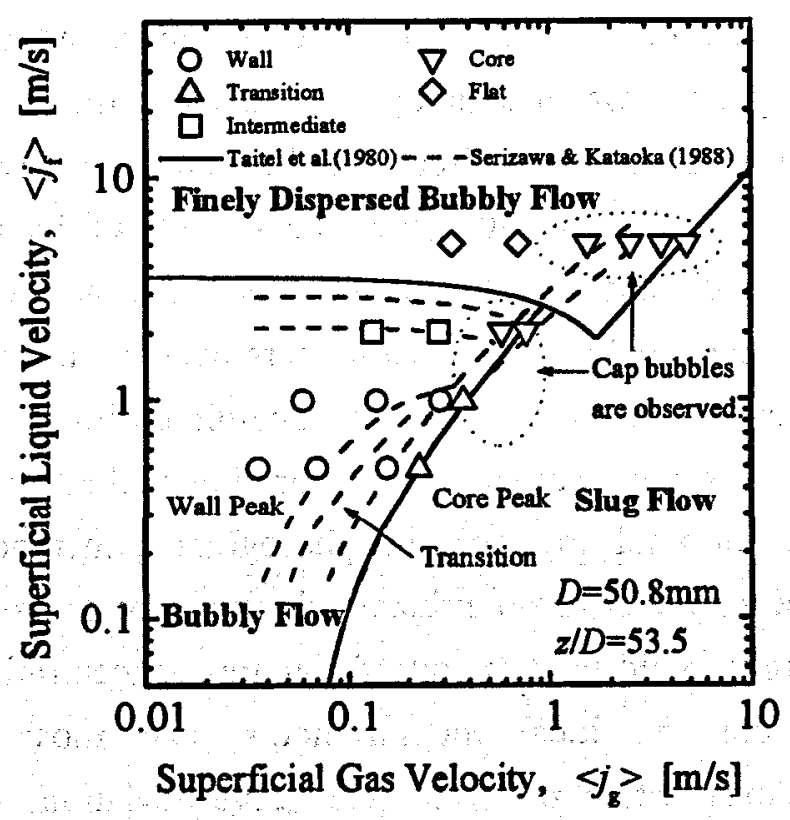

Figure 5.1. Flow conditions in the experiment using a $50.8 \mathrm{~mm}$ diameter pipe. 
shown in Fig.5.1, the observed void distribution agreed with the phase distribution pattern transition boundaries by Serizawa and Kataoka (1988). It should be noted here that the void fraction increased along the axial direction on the order of $20 \sim 40 \%$ between $z / D=6.00$ and 53.5 in the present experimental conditions due to the pressure reduction. This led to a continuously developing flow along the flow direction.

In the bubbly-to-slug flow transition measurements, bubbles could be separated into either a cap bubble or a small bubble based on the double-sensor probe signals (Leung et al., 1995). The determination whether detected bubbles were cap bubbles or not was performed based upon the chord length of bubbles. According to Clift's shape regime map for bubbles (Clift et al., 1978), the boundary between ellipsoidal and spherical-cap bubbles is given by $E o=40$, which corresponds to the bubble diameter of $17.2 \mathrm{~mm}$ at $20^{\circ} \mathrm{C}$. In the present experiment, when local bubble chord lengths exceeded $20 \mathrm{~mm}$, bubbles were considered as cap bubbles.

For the flow region where no cap bubbles appeared, local measurements of void fraction, interfacial area concentration, and interfacial velocity were successfully conducted by using the double sensor probe method (Revankar and Ishii, 1992; Hibiki et al., 1998a). A conductivity probe was held and positioned along the radius of the pipe using a traversing mechanism which mounted directly to the pipe flange. Data was taken at three different axial locations of $z / D=6.00,30.3$, and 53.5 as well as fifteen radial positions from $r / R=0$ to 0.95 . Here, one-dimensional flow parameters, that is, area averaged quantities can be obtained by integrating the local flow parameters over the flow channel. In order to verify the measurement accuracy, the obtained one-dimensional flow parameters were compared with those measured by other cross-calibration methods such as the single-fixed beam $\gamma$-densitometer for void fraction, the photographic method for interfacial area concentration, and the rotameter for interfacial velocity. Good agreements were obtained between the area averaged void fraction, interfacial area concentration, and the interfacial velocity obtained from the local measurements, and those measured by each cross-calibration methods within the average relative deviations of $\pm 5.74, \pm 6.95$, and $\pm 12.4 \%$, respectively (Hibiki et al., 1998a; 1998b).

Even in the flow region where cap bubbles appear, the double sensor probe could be used to measure local measurements of void fraction, interfacial area concentration, and interfacial velocity of small bubbles as well as local measurements of void fraction of cap bubbles. On the other hand, the multi-sensor probe should be used to measure local interfacial area concentration of cap bubbles instead of the double sensor probe. Although 
the multi-sensor probe method (Revankar and Ishii, 1993; Kim et al., 1998; 1999) has been developed recently, a rigorous data base has not been available so far. Since the multi-sensor probe was not available in this experiment, the one-dimensional interfacial area concentration of cap bubbles is approximately estimated here by the signals from the double sensor probe as follows.

According to Clift et al. (1979), the shape of cap bubbles can be closely approximated as a segment of a sphere. For $R e>150$, the rear or base is quite flat, through sometimes irregular, and the wake angle of the rising cap bubble in a stagnant fluid is nearly $50^{\circ}$. Recently, it was reported that the wake angle of the rising cap bubble in a forced convective fluid in a round pipe is about $90^{\circ}$ (Morita et al., 1999). Here, the shape of the cap bubble is approximated as a semi-sphere. The cap bubble generally rises faster than a spherical/distorted bubble. However, as the liquid velocity increases, the rise velocity difference between them would come to be negligible. Thus, the rise velocity of the cap bubble is taken to be the same as the total gas velocity as

$$
\left\langle\left\langle v_{z}\right\rangle\right\rangle=\left\langle\left\langle v_{z, 1}\right\rangle\right\rangle=\left\langle\left\langle v_{z, 2}\right\rangle\right\rangle
$$

The error due to this assumption would be less than $10 \%$ for a superficial liquid velocity of $2 \mathrm{~m} / \mathrm{s}$, a void fraction of $25 \%$, and a rise velocity difference of $30 \mathrm{~cm} / \mathrm{s}$ by conservative estimates. The one-dimensional interfacial area concentration of cap bubbles, $\left\langle a_{i, 2}\right\rangle$, can be estimated by the following equation with measured superficial gas velocity, $\left\langle j_{\mathrm{B}}\right\rangle$, void fraction of cap bubble, $\left\langle\alpha_{2}\right\rangle$, and number of cap bubbles passing the probe per unit time, $N_{\mathrm{b}, 2}$.

$$
\left\langle a_{i, 2}\right\rangle=6.24\left\langle\alpha_{2}\right\rangle\left(\frac{N_{b, 2}}{\left\langle j_{g}\right\rangle D^{2}}\right)^{1 / 3}
$$

where $D$ is the pipe diameter. Sauter mean diameter, $D_{\mathrm{Sm}, \mathrm{k}}$, is calculated by the following relation:

$$
\left\langle D_{s m, k}\right\rangle=\frac{6\left\langle\alpha_{k}\right\rangle}{\left\langle a_{i, k}\right\rangle}
$$

Figure 5.2 shows the axial change of one-dimensional flow parameters such as void fraction, interfacial area concentration, and Sauter mean diameter of small and cap bubbles, which were measured at the flow conditions surrounded by the dotted line in Fig.5.1. Flow parameters except $\left\langle a_{\mathrm{i}, 2}\right\rangle$ and $\left\langle D_{\mathrm{Sm}, 2}\right\rangle$ were measured by the double sensor 
$-0-\langle j>=0.986 \mathrm{~m} / \mathrm{s},\langle j \mathrm{~kg}>0.321 \mathrm{~m} / \mathrm{s}$

$\cdots . \Delta \cdots<j>2.01 \mathrm{~m} / \mathrm{s},<j,>-0.471 \mathrm{~m} / \mathrm{s}$

- o. $\langle j\rangle-2.01 \mathrm{~m} / \mathrm{s},\left\langle\mathrm{j}_{\mathrm{z}}>-0.624 \mathrm{~m} / \mathrm{s}\right.$

$-<<>-5.00 \mathrm{~m} / \mathrm{s},<j,>-1.11 \mathrm{~m} / \mathrm{s}$

$-<-<>-5.00 \mathrm{~m} / \mathrm{m}, j_{20}>-1.79 \mathrm{~m} / \mathrm{s}$

$\rightarrow-\langle j>-5.00 \mathrm{~m} / \mathrm{s},\langle j \mathrm{j},>-2.87 \mathrm{~m} / \mathrm{s}$

$\rightarrow-\left\langle j>-5.00 \mathrm{~m} / \mathrm{s},<j_{c t}>-3.90 \mathrm{~m} / \mathrm{s}\right.$
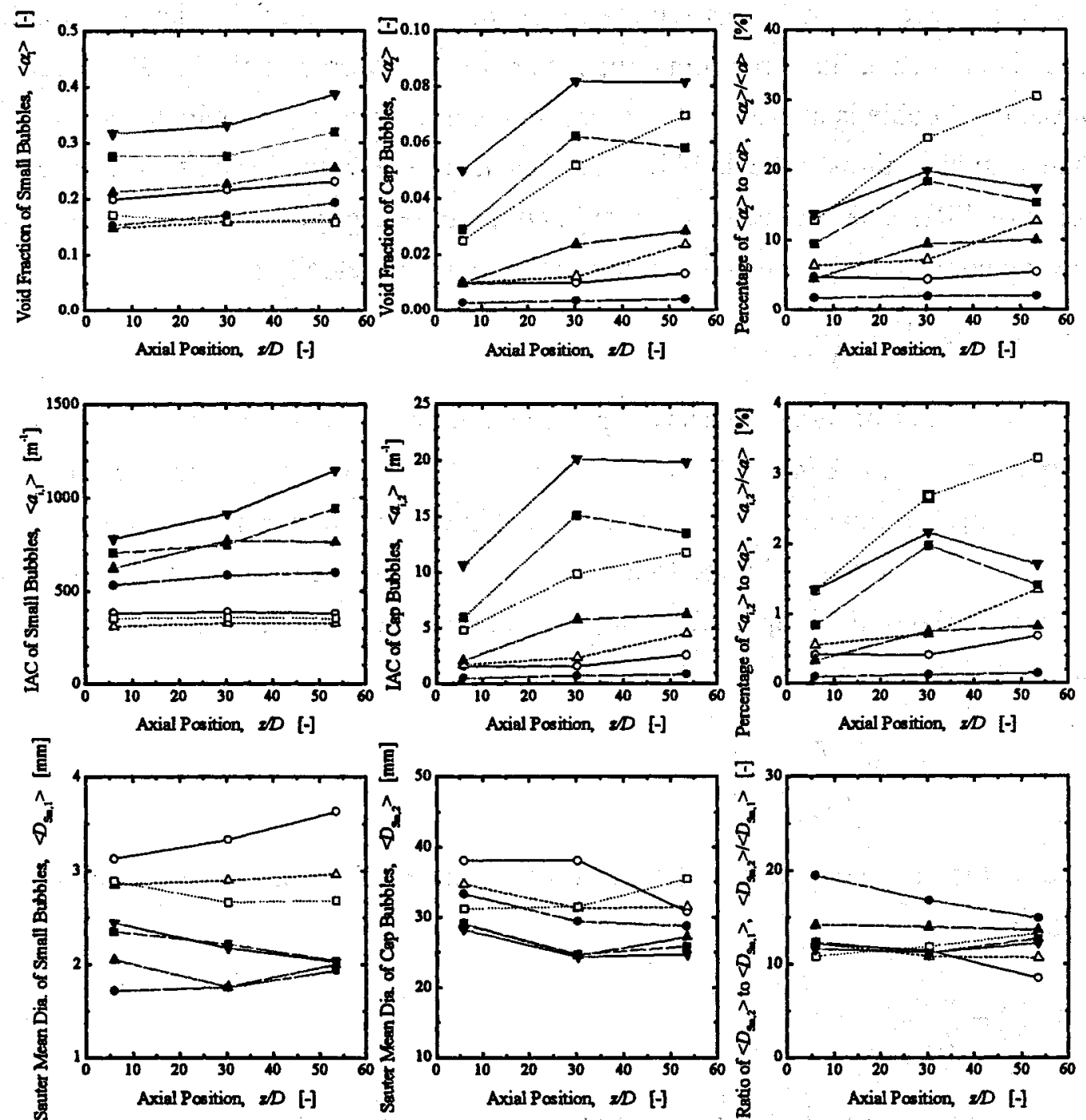

Figure 5.2. Axial development of one-dimensional void fraction, interfacial area concentration, and Sauter mean diameter of small and cap bubbles. 
probe method. Although total void fraction increases along the flow direction due to the pressure reduction, the increase rate in the void fraction of small bubbles, $\left\langle\alpha_{1}\right\rangle$, is diminished by the void fraction interchange between groups I and II. Void fractions of cap bubbles, $\left\langle\alpha_{2}\right\rangle$, are increased along the axial direction except for $\langle j p\rangle=5.00 \mathrm{~m} / \mathrm{s}$ and high void fraction. In the flow conditions, the formation and disintegration of cap bubbles appears to be in an equilibrium state. Since the interfacial area concentration is directly proportional to the area-averaged void fraction, the interfacial area changes along the flow direction display the same behavior as their respective void fraction changes for constant Sauter mean diameters. It should be noted here that the percentage of $\left\langle a_{i, 2}\right\rangle$ to $\left\langle a_{i}\right\rangle$ is less than about $3 \%$ even for $\left\langle\alpha_{2}\right\rangle \mid\langle\alpha\rangle \approx 30 \%$. This suggests that total interfacial area concentration is mainly attributed to small bubbles in the flow conditions. Therefore, the estimation error of $\left\langle a_{i, 2}\right\rangle$ by Eq.(5.2) does not affect the accuracy of $\left\langle a_{\mathrm{i}}\right\rangle$ in the flow conditions significantly. However, this does not mean that the introduction of the two-group interfacial area transport equations is not needed to explain the interfacial area transport. The interchange of void fraction between groups I and II markedly varies the interfacial area concentration of group $I,\left\langle a_{i, 1}\right\rangle$. The interfacial area concentration change would be approximately in proportion to the void fraction interchange. The Sauter mean diameters are found to be $1.5-3.5 \mathrm{~mm}$ and $25-40 \mathrm{~mm}$ for small and cap bubbles, respectively.

It should be noted here that there may be an inconsistency between the model derivation and the experimental condition in the strict sense. Since the models proposed in this study were derived under an implicit assumption of bubbles in an infinite medium, the present models did not take the effect of a channel wall on the flow dynamics into account. Therefore, the models might be appropriate basically for a bubbly flow in a container much larger than bubbles, where the effects of the dynamics in the cross stream direction would be confined to regions near a wall. In the experimental condition to be used for the evaluation of the models, diameters of the pipe, small bubbles, and cap bubbles were 50.8 $\mathrm{mm}, \mathbf{1} .5-3.5 \mathrm{~mm}$, and $25-40 \mathrm{~mm}$, respectively. In such an experimental condition, not spherical cap bubbles but Taylor bubbles might be formed and the dynamics of the processes in the cross stream direction might not be negligible. However, models taking these effects into account would be very complicated and require adjustable parameters much more than the present models. In addition to this, it would be thought that the present preliminary models could represent the basic mechanisms of bubble coalescence and breakup and work even for a pipe flow approximately. Since the main objective of this 
study is to show the capability of the two-group interfacial area transport equations and available data sets are quite limited at present, data sets taken in the $50.8 \mathrm{~mm}$-diameter pipe will be used for the validation of preliminary models proposed in this study. Distortions due to the inconsistency between the model derivation and the experimental situation are adjusted by adjustable parameters. In a future study, the development of much sophisticated models and designed experiments should be performed by taking the effect of the wall on the flow dynamics into account. It should be noted here that a bubble belonging to the group II is called "cap bubble" in this study without distinguishing between a cap bubble and a Taylor bubble.

\subsection{Predictions of Flow Parameters Used in Interfacial Area Transport Calculation}

In order to perform the interfacial area transport calculation, local pressure, superficial gas velocity, gas velocity (or $z$-component of the interfacial velocity), void fraction, gas velocity weighted by the interfacial area concentration, and bubble diameter should be given by either closure relations or measured values. For example, the system pressure can be calculated by using Lockhart-Martinelli's method (Lockhart and Martinelli, 1949) to estimate the two-phase frictional pressure loss. Good agreement was obtained between calculated and measured system pressures within the averaged relative deviation of $\pm 0.99 \%$ (maximum relative error of $\pm 5.04 \%$ ) in the experiment. The drift flux model (Ishii, 1977) can be used for the predictions of the void fraction and the gas velocity. The predicted gas velocities agreed with ones measured in the experiments within the averaged relative deviation of $\pm 11.3 \%$.

To examine the validity of the modeled sink and source terms, flow parameters used in the calculation should be as accurate as possible. Therefore, empirical equations shown in Table 5.3 are used in this calculation. The functional form of the system pressure is obtained from the linear pressure reduction along the flow direction in the measured range. The functional form of the void fraction is determined from insignificant changes in gas velocity along the flow direction (less than $\pm 3 \%$ ). In the equations, $P_{0},\left\langle j_{g_{0}}\right\rangle,\left\langle\alpha_{0}\right\rangle$, and $A_{0}$ are the inlet pressure, the inlet superficial gas velocity, the inlet void fraction, and the pressure gradient along the flow direction, respectively. These values of $P_{0},\left\langle j_{B_{0}}\right\rangle$, $\left\langle\alpha_{0}\right\rangle$, and $A_{0}$ were determined by values measured at three axial locations. Table 5.4 shows typical values used in the interfacial area transport calculations to be presented in the next section. The expected estimation errors are also shown in Table 5.3. The empirical equations give better estimations of the pressure and the gas velocity rather than the models 
Table 5.3 Empiriral correlations used in the predictions of system pressure and flow parameters.

\begin{tabular}{|c|c|c|}
\hline Quantities & Empirical Correlations & $\begin{array}{c}\text { Prediction } \\
\text { Accuracy }\end{array}$ \\
\hline System Pressure & $P=\left\{P_{0}+A_{0} \cdot(z / D)\right\}$ & $\begin{array}{c}<.5 \% \\
(\text { Max.: }<1 \%)\end{array}$ \\
\hline Superficial Gas Velocity & $\left\langle j_{s}\right\rangle=\frac{\left\langle j_{g, 0}\right\rangle \cdot P_{0}}{P}$ & $10.2 \%$ \\
\hline Void Fraction & $\langle\alpha\rangle=\frac{\left\langle\alpha_{0}\right\rangle \cdot P_{0}}{P}$ & $9.64 \%$ \\
\hline $\begin{array}{c}\text { Gas Velocity } \\
\text { (Interfacial Velocity) }\end{array}$ & $\left\langle\left\langle v_{z}\right\rangle\right\rangle=\frac{\left\langle j_{g}\right\rangle}{\langle\alpha\rangle}=\frac{\left\langle j_{g, 0}\right\rangle}{\left\langle\alpha_{0}\right\rangle}=\left\langle\left\langle v_{z, 0}\right\rangle\right)$ & $2.93 \%$ \\
\hline $\begin{array}{c}\text { Gas Velocity Weighted by } \\
\text { Interfacial Area Concentration }\end{array}$ & $\left\langle\left\langle v_{z z}\right\rangle\right\rangle_{a} \equiv \frac{\left\langle v_{i z} a_{i}\right\rangle}{\left\langle a_{i}\right\rangle} \approx \frac{\left\langle v_{z} \alpha\right\rangle}{\langle\alpha\rangle}=\left\langle\left\langle v_{z}\right\rangle\right\rangle$ & $1.40 \%$ \\
& $\left\langle\left\langle v_{i z}\right\rangle\right\rangle \equiv \frac{\left\langle v_{i z} \alpha\right\rangle}{\langle\alpha\rangle} \approx \frac{\left\langle v_{z} \alpha\right\rangle}{\langle\alpha\rangle}=\left\langle\left\langle v_{z}\right\rangle\right\rangle$ & \\
\hline
\end{tabular}

Table 5.4 Typical initial values used in the interfacial area transport calculation.

\begin{tabular}{|c|c|c|c|c|c|}
\hline \multicolumn{2}{|c|}{ Quantities } & Case II & Case II & Case III & Case IV \\
\hline \multicolumn{2}{|c|}{ Inlet Superficial Gas Velocity, $\left\langle j_{\mathrm{g}, 0}>[\mathrm{m} / \mathrm{s}]\right.$} & 0.321 & 0.624 & 1.11 & 3.90 \\
\hline \multicolumn{2}{|c|}{$\begin{array}{c}\text { Superficial Liquid Velocity, } \\
<j p[\mathrm{~m} / \mathrm{s}]\end{array}$} & 0.986 & 2.01 & 5.00 & 5.00 \\
\hline \multicolumn{2}{|c|}{$\begin{array}{c}\text { Inlet Pressure } \\
\boldsymbol{P}_{\mathbf{0}}[\mathrm{MPa}]\end{array}$} & 0.128 & 0.129 & 0.142 & 0.170 \\
\hline \multicolumn{2}{|l|}{$\begin{array}{l}\text { Pressure Gradient } \\
\qquad A_{0}[\mathrm{kPa}]\end{array}$} & -0.377 & -0.377 & -0.624 & -0.754 \\
\hline \multicolumn{2}{|l|}{$\begin{array}{l}\text { Inlet Void Fraction } \\
\qquad<\alpha_{0}>[-]\end{array}$} & 0.205 & 0.192 & 0.151 & 0.357 \\
\hline \multirow{2}{*}{$\begin{array}{c}\text { Void Fraction } \\
<\alpha_{k, z / D=6.00}>\left[\mathrm{m}^{-1}\right]\end{array}$} & Group I & 0.199 & 0.170 & 0.152 & 0.317 \\
\hline & Group II & 0.0100 & 0.0250 & 0.00274 & 0.0500 \\
\hline \multirow{2}{*}{ 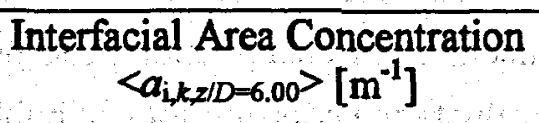 } & Group I & 382 & 354 & 532 & 778 \\
\hline & Group II & 1.61 & 4.84 & 0.481 & 10.5 \\
\hline
\end{tabular}


such as the Lockhart-Martinelli's equation and the drift flux model.

The interfacial area concentrations measured at the first measuring station such as $z / D=6.00$ for $D=50.8 \mathrm{~mm}$ are used as initial values. It should be noted here that the accuracy of the initial value may affect the final result of the calculation on the order of the accuracy of the initial value. Here, the Sauter mean diameters are calculated by Eq.(5.3). Due to the spherical bubble assumption for group I, we set $\left\langle D_{b, 1}\right\rangle \approx\left\langle D_{\mathrm{Sm}}\right\rangle$ in this calculation. Since a uniform bubble size is assumed, the area-averaged bubble interfacial velocity weighted by interfacial area concentration and the area-averaged bubble interfacial velocity weighted by void fraction can be given by:

$$
\left\langle\left\langle v_{i z}\right\rangle\right\rangle_{a} \equiv \frac{\left\langle v_{i z} a_{i}\right\rangle}{\left\langle a_{i}\right\rangle} \approx \frac{\left\langle v_{z} \alpha\right\rangle}{\langle\alpha\rangle}=\left\langle\left\langle v_{z}\right\rangle\right\rangle \text { and }\left\langle\left\langle v_{i z}\right\rangle\right) \equiv \frac{\left\langle v_{i z} \alpha\right\rangle}{\langle\alpha\rangle} \approx \frac{\left\langle v_{z} \alpha\right\rangle}{\langle\alpha\rangle}=\left\langle\left\langle v_{z}\right\rangle\right\rangle \text {. }
$$

As shown in Table 5.3, the area-averaged bubble interfacial velocities weighted by the interfacial area concentration and the void fraction agreed with the area-averaged velocity of the center of the volume of the bubble weighted by void fraction within the average relative deviation of $\pm 1.40 \%$.

Since measured values of the frictional pressure loss are not available, the Lockhart-Martinelli's method (1949) with Chisholm's equation (1967) is used to estimate the two-phase frictional pressure loss in the calculation of the energy dissipation given by Eq.(4.36). The prediction error of this model will be corrected by the adjustable parameters, $\Gamma_{\mathrm{RC}, 1^{\circ}}, \Gamma_{\mathrm{T}, 1}{ }^{\circ}$, and so on. The calculation of the interfacial area concentration change along the flow direction is performed by a finite difference method. The adjustable parameters and experimental coefficients used in the calculation are listed in Table 5.5. They are determined experimentally to fit the data. The propagated error due to the uncertainty induced by the component models listed in Table 5.3 is estimated to be $14.3 \%$.

\subsection{Contributions of Bubble Coalescence, Breakup and Expansion to the One-group Interfacial Area Transport}

When no cap bubbles are observed, the one-group interfacial area transport equation can be used to predict the interfacial area concentration change along the flow direction (Hibiki and Ishii, 1999b; 2000a). In order to evaluate the contributions of bubble coalescence, breakup, and expansion to the interfacial area transport, typical changes of interfacial area concentration due to each mechanism along axial position are shown in Figs.5.3 and 5.4. The changes of the system pressure, the void fraction and the Sauter 
Table 5.5 Summary of the adjustable valuables to predict the interfacial area concentration.

\begin{tabular}{|c|c|c|}
\hline Group & $\begin{array}{l}\text { Adjustable } \\
\text { Valuables }\end{array}$ & $\begin{array}{l}\text { Equations and database used for the determination of the } \\
\text { adjustable valuables. }\end{array}$ \\
\hline \multirow[t]{2}{*}{ Group I } & $\begin{array}{c}\Gamma_{\mathrm{RC}, 1}{ }^{\circ}=0.351 \\
\Gamma_{\mathrm{Tl}, 1}{ }^{\circ}=1.12\end{array}$ & $\begin{array}{l}\text { One-Group IAC Transport Equation, } 43 \text { Data sets (Hibiki } \\
\text { et al., 1998b; Hibiki and Ishii, 1999) }\end{array}$ \\
\hline & $\begin{aligned} K_{\mathrm{RC}, 1}{ }^{\circ} & =0.258 \\
K_{\mathrm{TH}, 1} & =6.85\end{aligned}$ & $\begin{array}{l}\text { One-Group IAC Transport Equation, } 43 \text { Data sets (Hibiki } \\
\text { et al., 1998b; Hibiki and Ishii, 1999) }\end{array}$ \\
\hline \multirow[t]{2}{*}{ Inter-group } & $\begin{aligned} \Gamma_{\mathrm{WE}, 12}{ }^{\circ} & =24.9 \\
\Gamma_{\mathrm{TL}, 12}{ }^{\circ} & =317\end{aligned}$ & Void Transport Equation, 7 Data sets (Hibiki et al., 1998b) \\
\hline & $\begin{aligned} K_{\mathrm{WE}, 12}{ }^{\circ} & =0.460 \\
K_{\mathrm{TL}, 12}{ }^{\circ} & =11.7\end{aligned}$ & $\begin{array}{l}K_{\mathrm{WE}, 12}{ }^{\circ}=1.78 \times K_{\mathrm{RC}, 1}{ }^{\circ} \\
K_{\mathrm{TL}, 12}{ }^{\circ}=3.85 \times K_{\mathrm{TL}, 1}{ }^{\circ}\end{array}$ \\
\hline \multirow[t]{2}{*}{ Group II } & $\begin{aligned} \Gamma_{\mathrm{WE}, 2}{ }^{\circ} & =63.7 \\
\Gamma_{\mathrm{TL}, 2}{ }^{\circ} & =4.26\end{aligned}$ & $\begin{array}{l}\text { Two-Group IAC Transport Equations, } 7 \text { Data sets (Hibiki } \\
\text { et al., 1998b) }\end{array}$ \\
\hline & $\begin{aligned} K_{\mathrm{WE}, 2}{ }^{\circ} & =0.258 \\
K_{\mathrm{TL}, 2}{ }^{\mathrm{O}} & =6.85\end{aligned}$ & $\begin{array}{l}K_{\mathrm{WE}, 2}{ }^{\circ}=K_{\mathrm{RC}, 1}{ }^{\circ} \\
K_{\mathrm{TI}, 2}{ }^{\circ}=K_{\mathrm{TI}, 1}{ }^{\circ}\end{array}$ \\
\hline
\end{tabular}

mean diameter along the flow direction are also shown in Fig.5.5.

For the case of low liquid velocity and low void fraction such as $\left\langle j_{\mathrm{f}}\right\rangle=0.491 \mathrm{~m} / \mathrm{s}$ and $\left\langle j_{\mathrm{g}, 0}\right\rangle=0.0275 \mathrm{~m} / \mathrm{s}\left(\left\langle\alpha_{z / D=53.5}\right\rangle=4.90 \%\right)$, the change of the interfacial area concentration predicted by the derived one-group models (Eqs.(4.4) and (4.24)) suggests that bubble coalescence due to random collision between bubbles and bubble breakup due to random collision between bubbles and turbulent eddies are not marked (See Fig.5.3). For this flow condition, the bubble mean free path may not be large enough to cause bubble random collision because of the long distance between bubbles. The bubble coalescence and breakup efficiency are estimated to be 0.93 and $\sim 0$ by Eqs.(4.2) and (4.22), respectively. For the small turbulence fluctuation, the bubble contact time is relatively long, resulting in high coalescence efficiency, whereas the turbulent eddy may not have enough energy to disintegrate the bubbles. This leads to a major role of the bubble expansion due to the pressure reduction along the axial direction in the interfacial area transport. As a result, the interfacial area concentration increases along the axial direction mainly due to the pressure reduction. Reasonably good agreement is obtained between predicted and measured interfacial area concentrations.

For the case of low liquid velocity and high void fraction such as $\left\langle j_{\mathrm{P}}\right\rangle=0.491 \mathrm{~m} / \mathrm{s}$ and $\left\langle j_{\mathrm{g} 0}\right\rangle=0.190 \mathrm{~m} / \mathrm{s}\left(\left\langle\alpha_{z / D}=53.5\right\rangle=25.9 \%\right)$, the increase of void fraction would enhance the rate of collision between bubbles as well as that between bubbles and turbulent eddies (see 


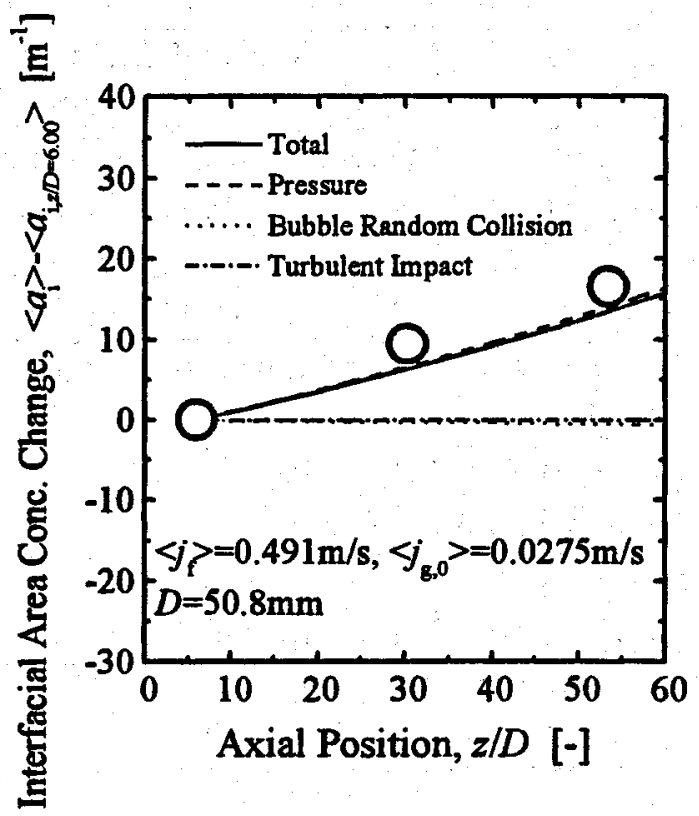

Figure 5.3. Comparison of measured interfacial area concentration change along the flow direction with one predicted by the one-group interfacial area transport equation $\left(\langle j \mathrm{p}\rangle=0.491 \mathrm{~m} / \mathrm{s},\left\langle j_{\mathrm{g}, 0}\right\rangle=0.0275 \mathrm{~m} / \mathrm{s}\right)$.

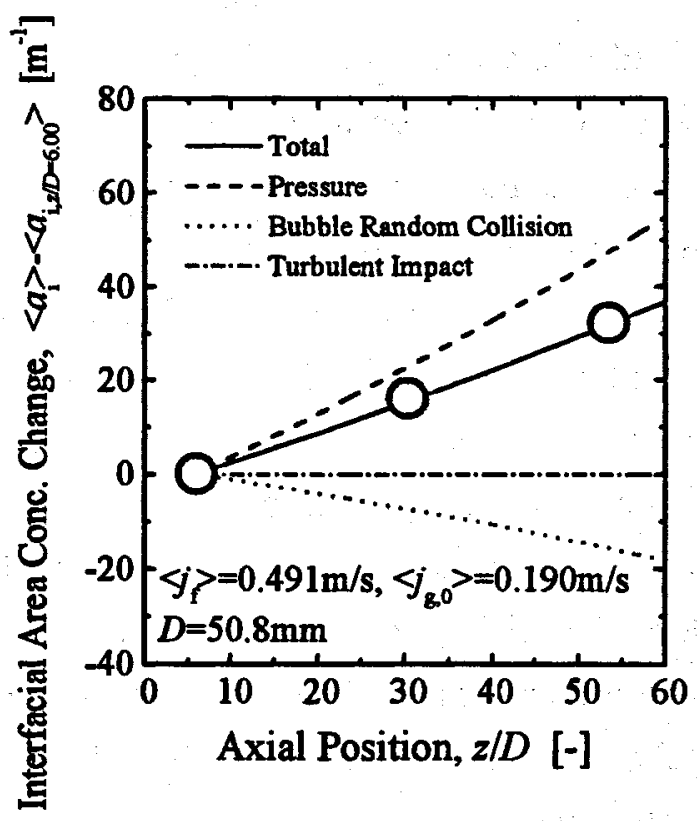

Figure 5.4. Comparison of measured interfacial area concentration change along the flow direction with one predicted by the one-group interfacial area transport equation $\left(\left\langle j_{\mathrm{p}}\right\rangle=0.491 \mathrm{~m} / \mathrm{s},\left\langle j_{\mathrm{g}, 0}\right\rangle=0.190 \mathrm{~m} / \mathrm{s}\right)$. 


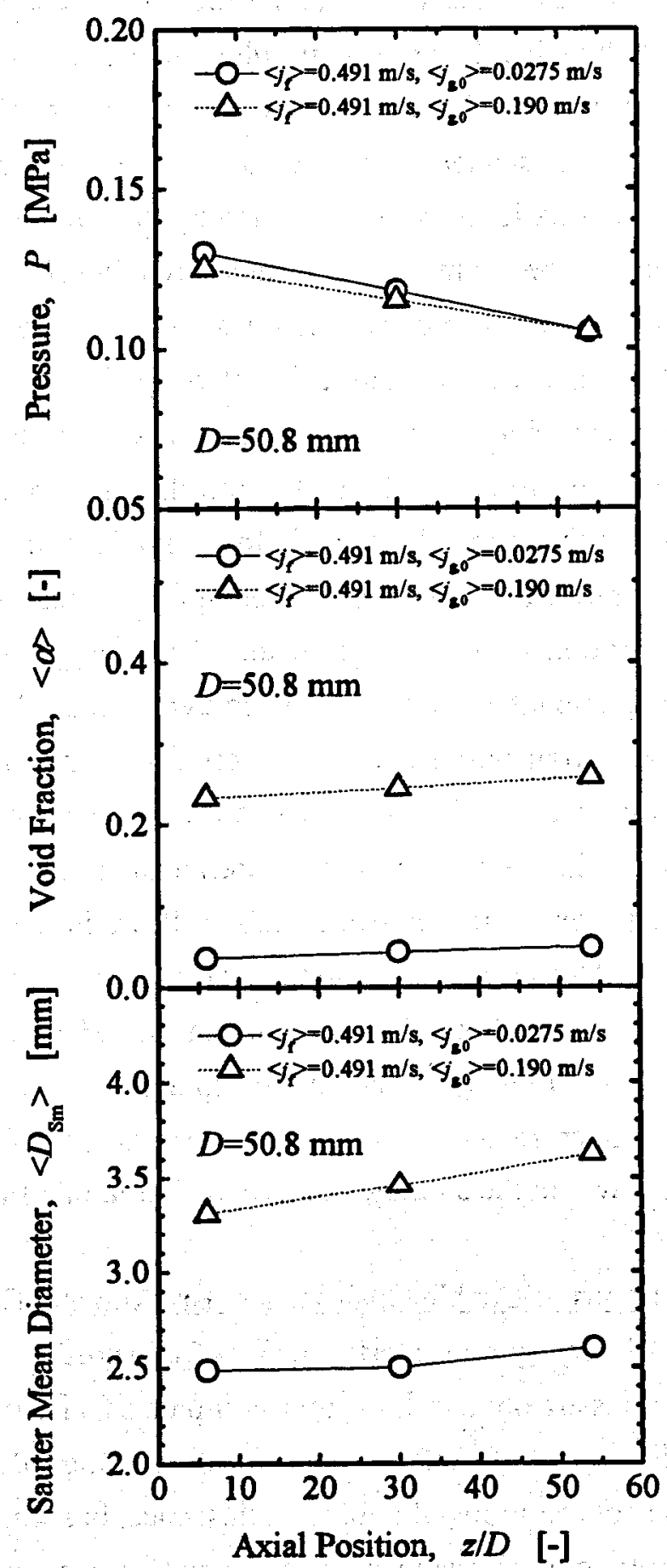

Figure 5.5. Examples of axial change of system pressure, void fraction, and Sauter mean diameter. 
Appendix A). The enhancement of the bubble coalescence along the flow direction tends to decrease the interfacial area concentration along the flow direction. On the other hand, the increase rate of the bubble breakup due to turbulent impact is not marked because of the gradual decrease of eddy number with increasing void fraction, and the low bubble breakup efficiency $\left(\lambda_{\pi, 1} \sim 0\right)$. As a consequence, the increase of the interfacial area concentration along the flow direction is smaller than that expected by the bubble expansion.

Figure 5.6 shows the comparison of measured interfacial area concentration changes along the flow direction with calculated ones. In the figure, solid and broken lines indicate the interfacial area concentrations calculated by the one-group and two-group interfacial area transport equations, respectively. The one-group interfacial area transport equation can reproduce the proper trends of the interfacial area concentration changes along the flow direction in the range of relatively low void fraction. However, as the void fraction increases, the deviation between the one-group equation and the measured interfacial area concentration becomes significant. This suggests the necessity of the introduction of the two-group interfacial area transport equations. As shown in Fig.5.7, the one-group interfacial area transport equation can predict interfacial area concentrations (O) measured in a relatively low void fraction region within the average relative deviation of $\pm 9.51 \%$. As shown in Fig.5.8, the one-group interfacial area transport equation is also compared with three data for vertical air-water bubbly flows in a $38.1 \mathrm{~mm}$-inner diameter pipe (Grossetete, 1995). Although Grossetete took 11 data sets over the range of the void fraction from $4.4 \%$ to $33 \%$, three data sets taken in relatively low void fraction $\left(<\alpha_{z / D=155}><10 \%\right)$ were used to validate the one-group equation. The one-group equation can also reproduce the proper trend of the interfacial area transport along the flow direction and agrees with the data within the average relative deviation of $\pm 14.6 \%$.

\subsection{Contributions of Bubble Coalescence, Breakup and Expansion to \\ Two-group Interfacial Area Transport}

When cap bubbles are observed, the two-group interfacial area transport equations should be used to predict the interfacial area concentration change along the flow direction. In order to evaluate the contributions of bubble coalescence, breakup and expansion to the interfacial area transport, typical changes of interfacial area concentration due to each mechanism along axial position are shown in Figs.5.9-5.12.

For $\left\langle j_{\mathrm{p}}\right\rangle=0.986 \mathrm{~m} / \mathrm{s}$ and $\left\langle j_{\mathrm{g}, 0}\right\rangle=0.321 \mathrm{~m} / \mathrm{s}\left(\left\langle\alpha_{z / D=53.5}\right\rangle=23.1 \%\right)$, the change in the void fraction of small bubbles (Group I) predicted by the void fraction transport equation 

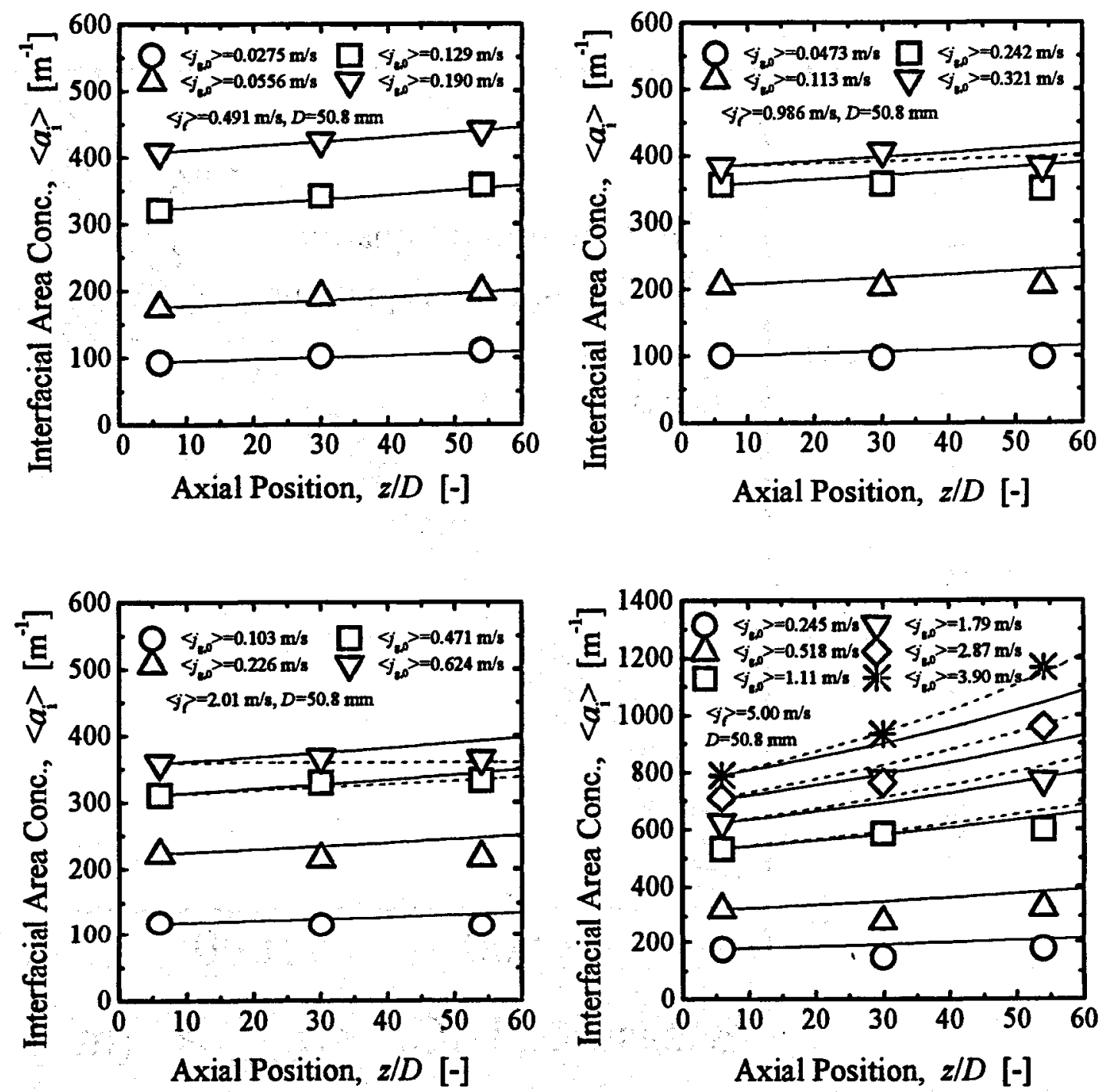

Figure 5.6. Prediction of axial change of one-dimensional interfacial area concentrations measured in a $50.8 \mathrm{~mm}$ diameter pipe by one-group (solid lines) and two-group (broken lines) interfacial area transport equations with modeled sink and source terms. 


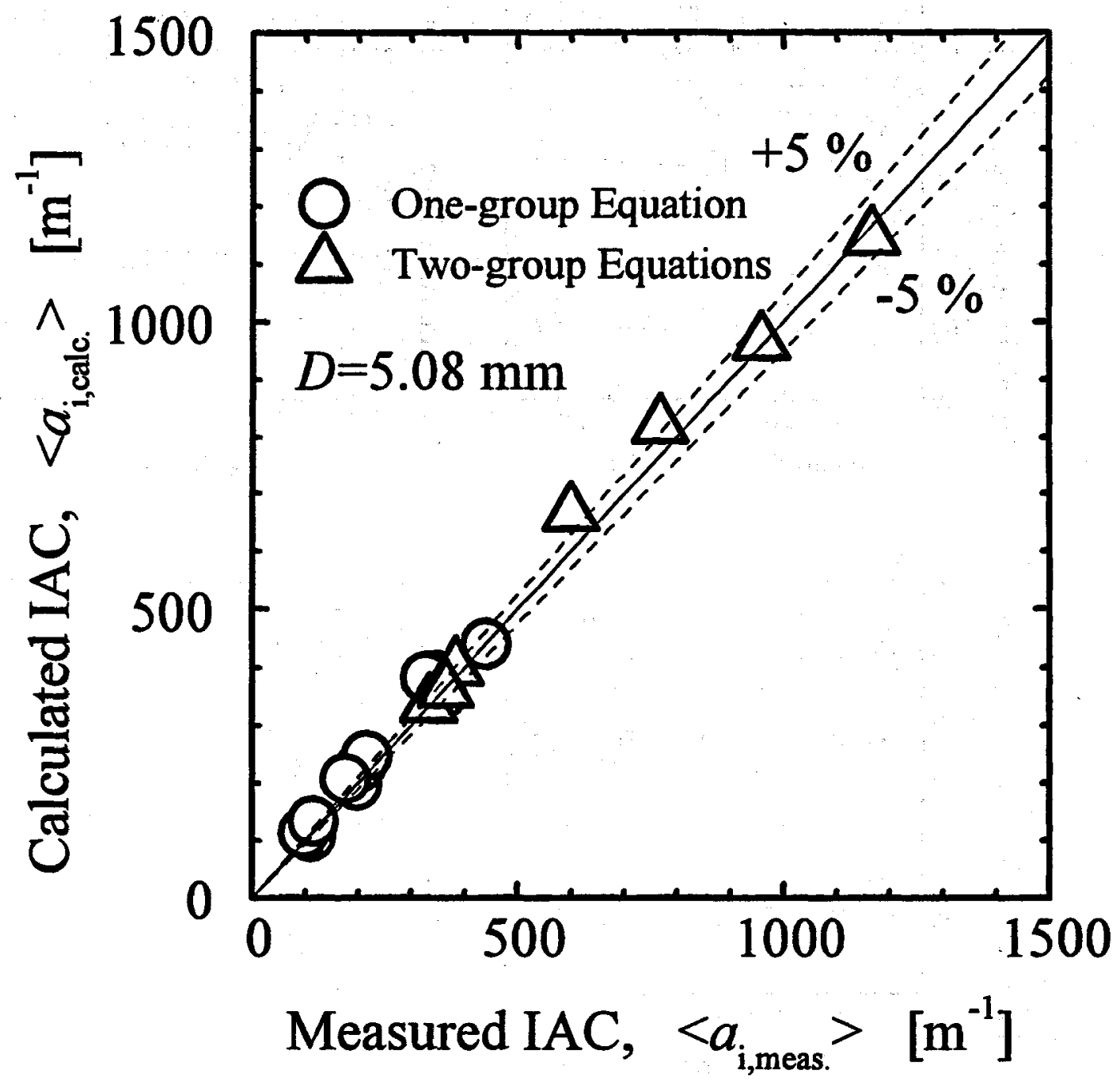

Figure 5.7. Comparison between measured and predicted interfacial area concentrations. 

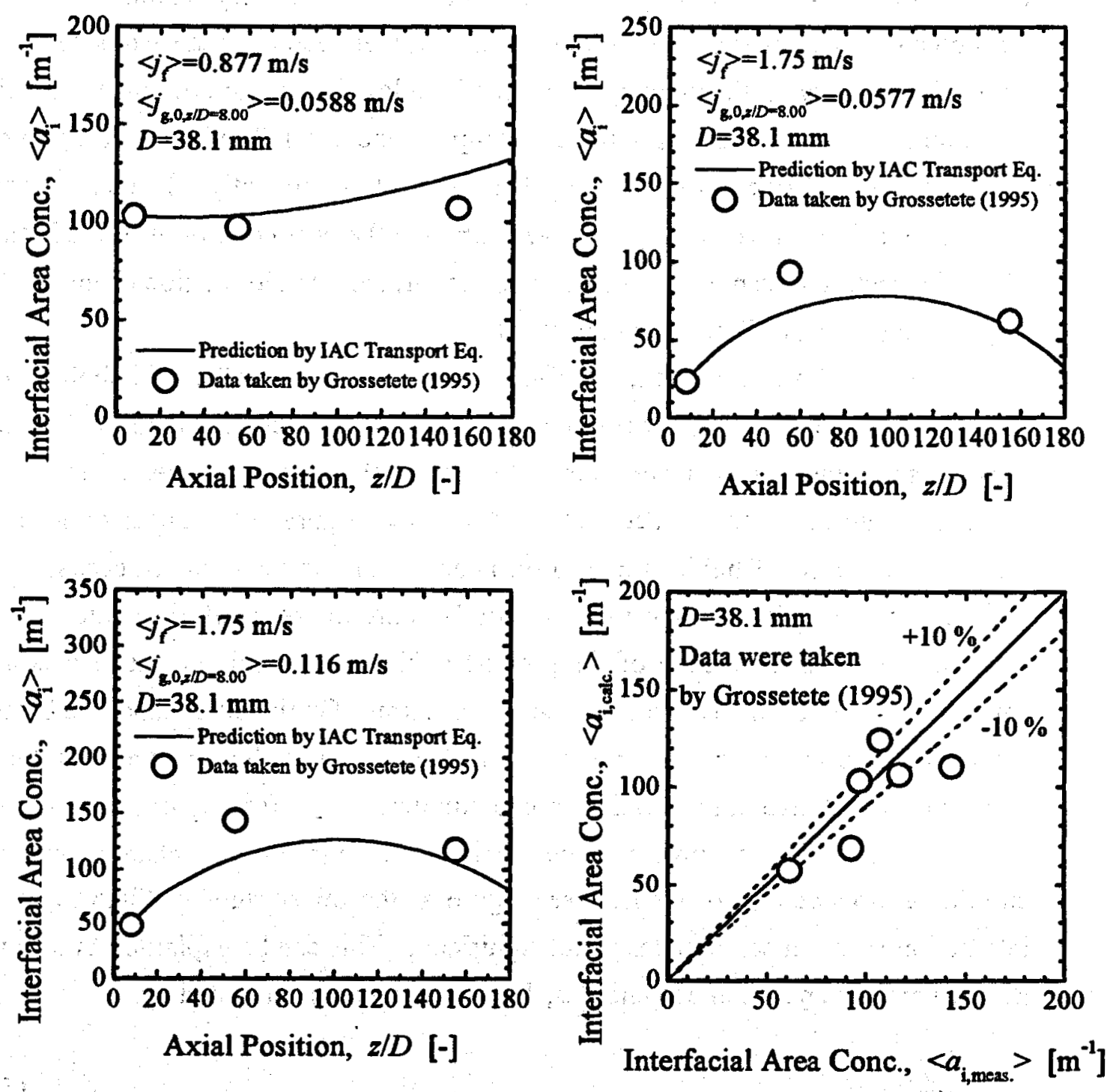

Figure 5.8. Comparison between one-group interfacial area transport equation and data taken by Grossetete (1995). 
with the derived models for the sink and source terms shows that the increase in void fraction, $\left\langle\alpha_{1}\right\rangle$, along the flow direction is mainly attributed to the pressure reduction. The interchange of the void fraction from group I to group II due to wake entrainment tends to decrease the void fraction slightly (see figure at the upper left in Fig.5.9). The transport of cap bubbles to small bubbles due to turbulent impact is very small. This would be attributed to low frequency of cap bubbles and turbulent eddies because of small number of large eddies acting on the breakup of the cap bubbles. In addition to this, the breakup of a cap bubble into a smaller cap bubble (Group II) and a small bubble (Group I) does not transport the void fraction from group II to group I significantly. Thus, the transport of void fraction due to this mechanism is very small in the flow conditions tested in this study. The void fraction transport equation can predict measured void fraction changes, $\left\langle\alpha_{1}\right\rangle$ and $\left\langle\alpha_{2}\right\rangle$, along the flow direction satisfactorily.

As for the change in the interfacial area concentration of small bubbles, $\left\langle a_{i, 1}\right\rangle$, the two-group model suggests that the coalescence of small bubbles due to random collision, as well as the transport of small bubbles to cap bubbles due to wake entrainment decrease the rate of interfacial area concentration, $\left\langle a_{i, 1}\right\rangle$, (see figure at the upper right in Fig.5.9). The breakup of small bubbles due to turbulent impact is very small because of $\lambda_{\mathrm{Th}, 1} \approx 0$. Since the void fraction transport from group II to group I is small, the source term for the interfacial area concentration of the group-I bubble due to this mechanism is also very small in comparison with other sink and source terms. On the other hand, the breakup of a cap bubble into smaller cap bubbles due to turbulent impact slightly enhances the increase in the interfacial area concentration of cap bubbles, $\left\langle a_{i, 2}\right\rangle$, (see figure at the lower left in Fig.5.9). According to predicted interfacial area concentration changes due to wake entrainment between cap bubbles (see figure at the lower right in Fig.5.9), the bubble interaction between cap bubbles is not significant. This can be explained as follows. The average distance between cap bubbles, $L_{b}$, can be roughly estimated by:

$L_{b} \approx \frac{\left\langle v_{z, 2}\right\rangle}{N_{b, 2}}$,

In this flow condition, the ratio of the average distance between cap bubbles, $L_{\mathrm{b}}$, to the average chord length of cap bubbles, $D_{\mathrm{C}}$, is roughly 40 . This value of $L_{\mathrm{b}} / D_{\mathrm{C}}$ is much larger than $L_{\mathrm{WE}} / D_{\mathrm{C}}$ (3-4 by Otake et al. (1977); 5-7 by Tsuchiya et al. (1986)) at which the leading bubble begins to exert noticeable influence on the following one. Thus, almost no bubble coalescence due to wake entrainment between cap bubbles occurs. Eventually, the 

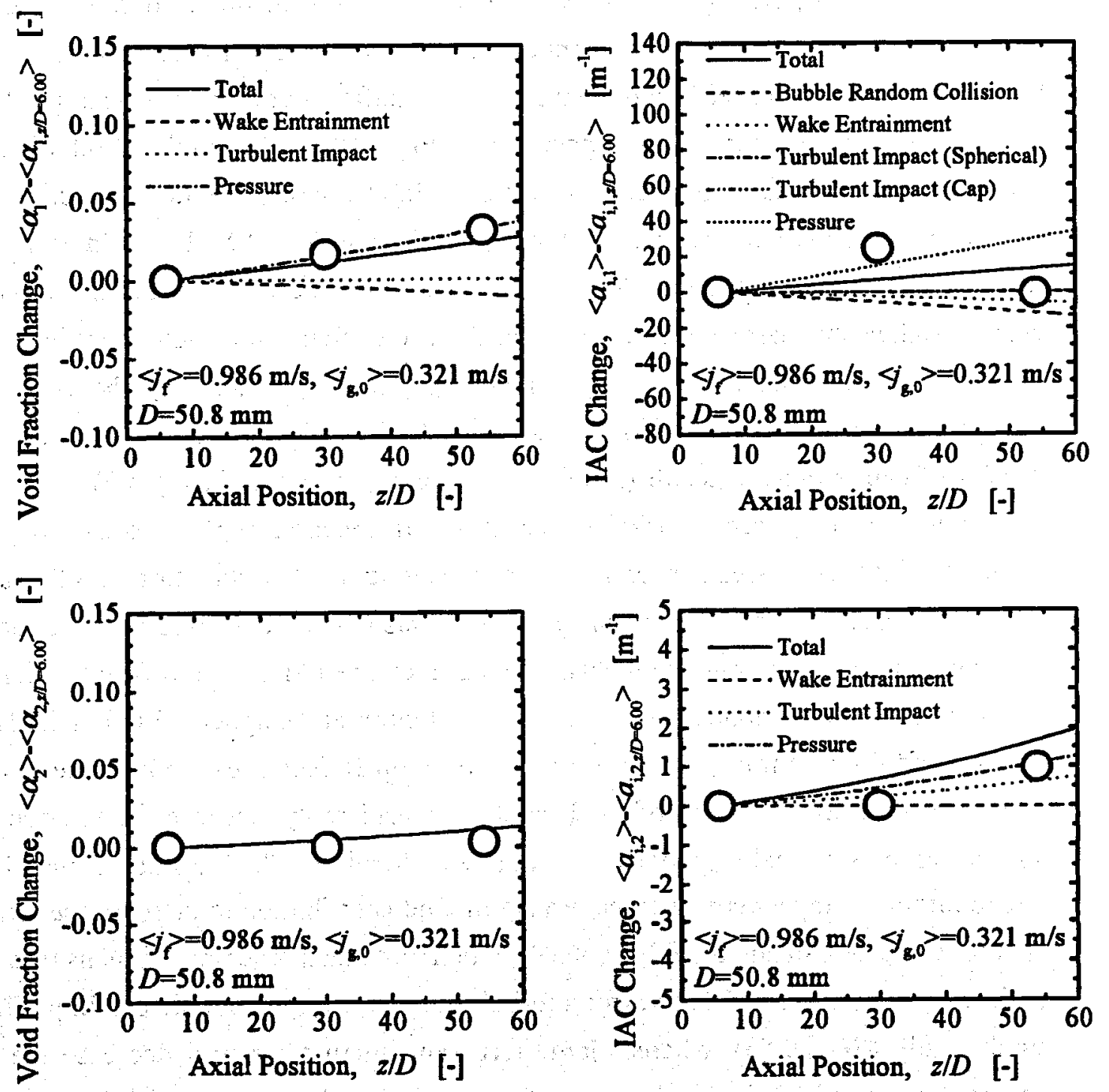

Figure 5.9. Predictions of axial development in void fraction and interfacial area concentration by the void fraction transport equation and two-group interfacial area transport equations $\left(\left\langle j_{p}>=0.986 \mathrm{~m} / \mathrm{s},\left\langle j_{g .0}>=0.321 \mathrm{~m} / \mathrm{s}\right)\right.\right.$. 
source and sink terms of the interfacial area concentration due to void transport from group II to group I, Eq.(4.30), and wake entrainment between cap bubbles, Eq.(4.20), play a minor role in the interfacial area transport.

For $\left\langle j_{\mathrm{f}}\right\rangle=2.01 \mathrm{~m} / \mathrm{s}$ and $\left\langle j_{g, 0}\right\rangle=0.624 \mathrm{~m} / \mathrm{s}\left(\left\langle\alpha_{z / D=53.5}\right\rangle=22.8 \%\right)$, the prediction by void fraction transport equation shows that the decrease in the void fraction of small bubbles, $\left\langle\alpha_{1}\right\rangle$, due to wake entrainment comes to be comparable to the increase in the void fraction due to pressure reduction, resulting in insignificant void fraction change along the flow direction (see figure at the upper left in Fig.5.10). The resulting interfacial area concentration exhibits a tendency to decrease slightly along the flow direction, which agrees with measurement (see figure at the upper right in Fig.5.10). It should be noted here that this tendency can not be produced by the one-group interfacial area transport equation with two adjustable parameters of $\Gamma_{\mathrm{RC}, 1}{ }^{\circ}$ and $\Gamma_{\mathrm{T}, 1}{ }^{\circ}$ (see figure at the lower left in Fig.5.10).

For $\left\langle j_{\mathrm{p}}\right\rangle=5.00 \mathrm{~m} / \mathrm{s}$ and $\left\langle j_{\mathrm{g}, 0}\right\rangle=1.11 \mathrm{~m} / \mathrm{s}\left(\left\langle\alpha_{2 / D}=53.5\right\rangle=20.0 \%\right)$, the void fraction transport along the flow direction is attributed to pressure reduction, since the number density of cap bubbles is small (see figure at the upper middle in Fig.5.2). In this flow condition, the one-group interfacial area transport equation appears to be sufficient to predict the interfacial area concentration change along the flow direction. As the gas flow rate is increased $\left(\left\langle j_{\mathrm{P}}\right\rangle=5.00 \mathrm{~m} / \mathrm{s}\right.$ and $\left\langle j_{\mathrm{g} 0}\right\rangle=3.90 \mathrm{~m} / \mathrm{s}\left(\left\langle\alpha_{2 / D=53.5}\right\rangle=44.2 \%\right)$ ), the formation of cap bubbles tends to promote the wake entrainment, resulting in significant void fraction transport from small bubbles to cap bubbles (see figures at the upper left in Fig.5.12). Such a large void fraction transport from group I to group II decreases the increase rate in the interfacial area concentration of small bubbles considerably, because the interfacial area concentration is basically proportional to the void fraction. The breakup of small bubbles due to turbulent impact comes to have a significant contribution to increase the interfacial area concentration of small bubbles, $\left\langle a_{i, 1}\right\rangle$, much more than other mechanisms (see figures at the upper right in Fig.5.12). Strong turbulence increases the bubble breakup efficiency significantly $\left(\lambda_{T, 1} \sim 0.55\right)$, whereas large turbulent fluctuations may decrease the bubble contact time, resulting in the decrease of the bubble coalescence efficiency $\left(\lambda_{\mathrm{RC}, 1} \sim 0.42\right)$. Thus, the total source rate of the interfacial area concentration of small bubbles becomes much higher than total sink rate.

In this flow condition, the average distance between cap bubbles $\left(L_{\mathrm{b}} / D_{\mathrm{c}} \sim 6\right)$ becomes much smaller than that for $\left\langle j_{\mathrm{p}}\right\rangle=0.986 \mathrm{~m} / \mathrm{s}$ and $\left\langle j_{\mathrm{g}, 0}\right\rangle=0.321 \mathrm{~m} / \mathrm{s}\left(L_{\mathrm{b}} / D_{\mathrm{c}} \sim 40\right)$. This value is critical to initiate the wake entrainment between cap bubbles. However, even though the number density of cap bubbles reduces to half due to wake entrainment, the 

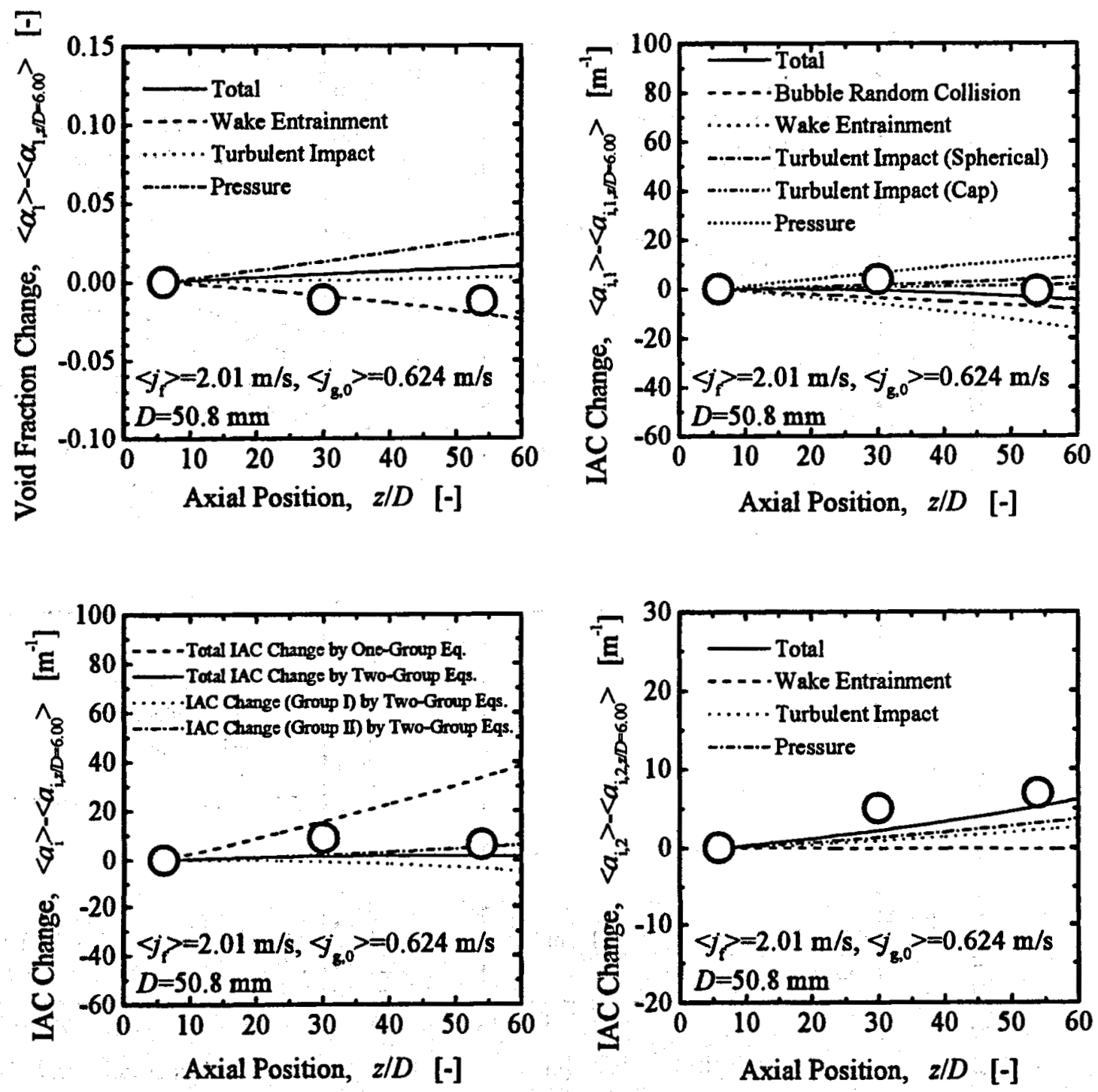

Figure 5.10. Predictions of axial development in void fraction and interfacial area concentration by the void fraction transport equation and two-group interfacial area transport equations $\left(\left\langle j p=2.01 \mathrm{~m} / \mathrm{s},\left\langle j_{g, 0}\right\rangle=0.624 \mathrm{~m} / \mathrm{s}\right)\right.$. 

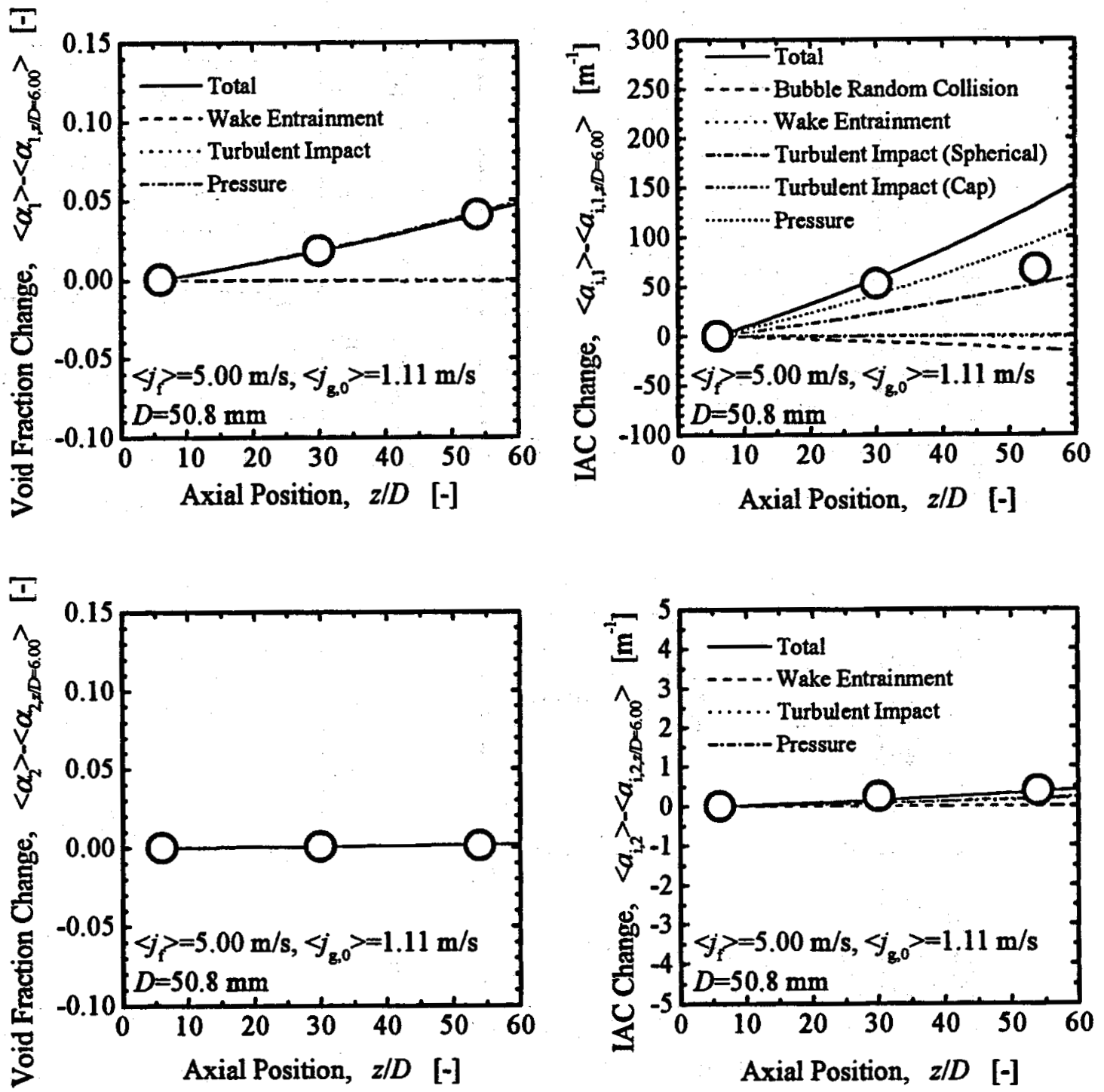

Figure 5.11. Predictions of axial development in void fraction and interfacial area concentration by the void fraction transport equation and two-group interfacial area transport equations $\left(\langle j \mathrm{p}\rangle=5.00 \mathrm{~m} / \mathrm{s},\left\langle j_{\mathrm{g}, 0}\right\rangle=1.11 \mathrm{~m} / \mathrm{s}\right)$. 

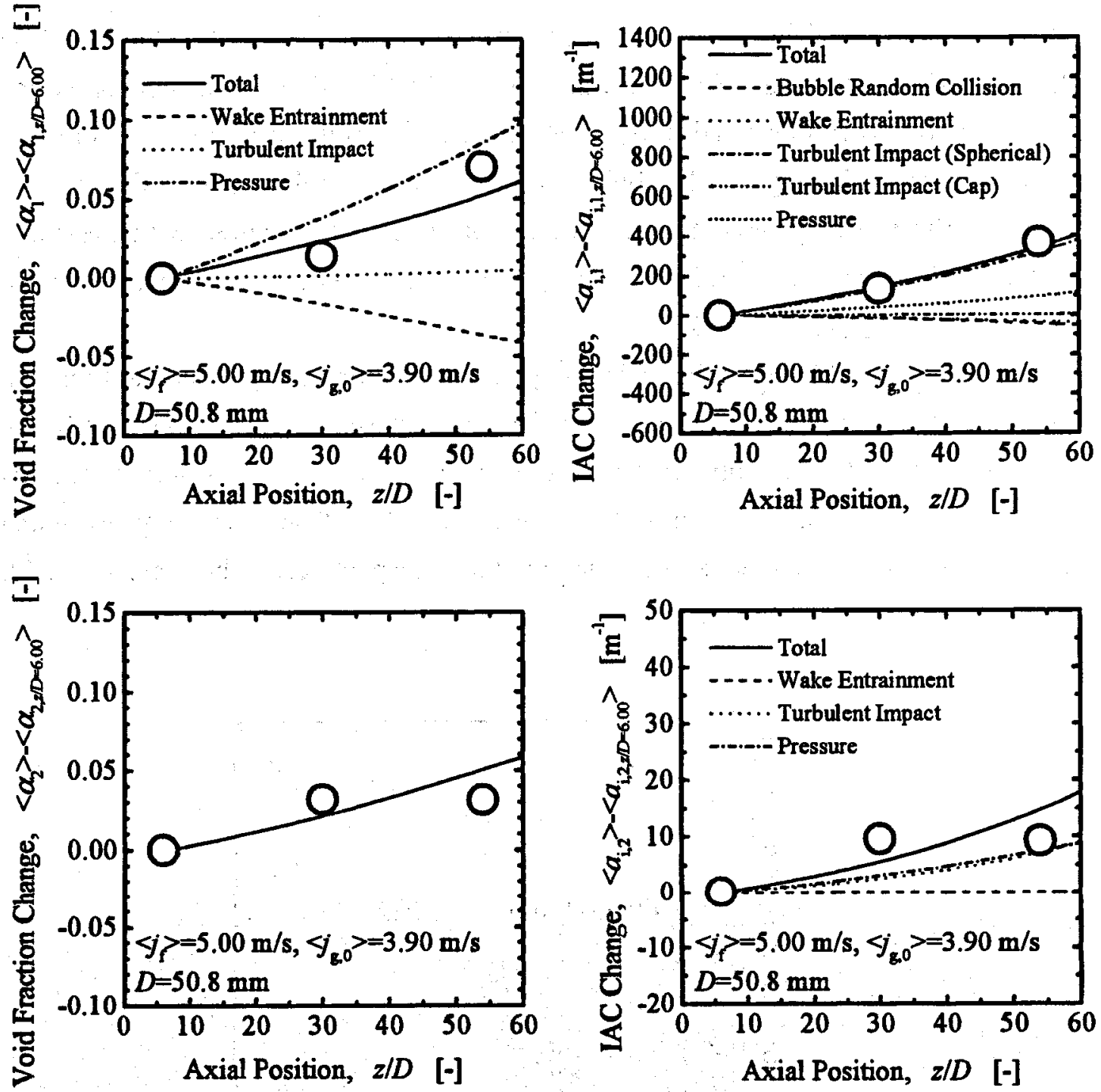

Figure 5.12. Predictions of axial development in void fraction and interfacial area concentration by the void fraction transport equation and two-group interfacial area transport equations $\left(\left\langle j \mathrm{p}>=5.00 \mathrm{~m} / \mathrm{s},\left\langle j_{\mathrm{g}, 0}\right\rangle=3.90 \mathrm{~m} / \mathrm{s}\right)\right.$. 
resulting decrease rate of the interfacial area concentration is about $20 \%$, which corresponds to $2.2 \mathrm{~m}^{-1}$ in $\left\langle a_{\mathrm{i}, 2}\right\rangle$ in this flow condition. Therefore, the coalescence of cap bubbles due to wake entertainment would play an important role in the interfacial area transport for flows with much higher void fraction such as slug flow.

As shown in Figs.5.6 and 5.7, the two-group interfacial area transport equations can predict the total interfacial area concentrations $(\triangle)$ measured at the bubbly-to-slug flow transition within a relative deviation of $\pm 3.61 \%$. Figure 5.13 compares predicted void fractions with measured ones. The void fraction transport equation can predict the void fractions of the group-I bubble within an average relative deviation of $\pm 4.35 \%$, whereas the predicted void fractions of the group-II bubble agree with measured ones within the average relative deviation of $\pm 31.2 \%$ corresponding to $\left|\left\langle\alpha_{2, \text { calc. }}\right\rangle-<\alpha_{2, \text { meas. }}\right\rangle \mid=0.919 \%$.

Figure 5.14 shows the scale analyses to determine the predominant terms among the sink and source terms for three flow conditions such as $\left\langle j_{\mathrm{f}}\right\rangle=0.986 \mathrm{~m} / \mathrm{s}$ and $\left\langle j_{\mathrm{g}, 0}\right\rangle=0.321 \mathrm{~m} / \mathrm{s}$ (upper figures), $\left\langle j_{\mathrm{p}}\right\rangle=2.01 \mathrm{~m} / \mathrm{s}$ and $\left\langle j_{\mathrm{g}, 0}>=0.624 \mathrm{~m} / \mathrm{s}\right.$ (middle figures), and $\left\langle j p=5.00 \mathrm{~m} / \mathrm{s}\right.$ and $\left\langle j_{g_{0}}\right\rangle=3.90 \mathrm{~m} / \mathrm{s}$ (lower figures). The left and right figures indicate the contributions of each sink term and source term, respectively, which are normalized by

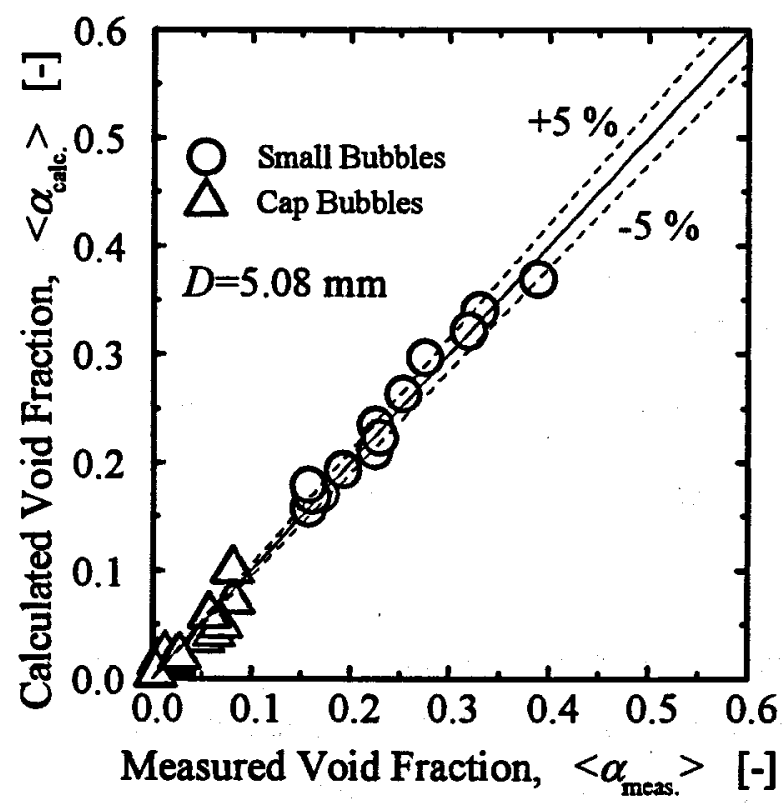

Figure 5.13. Comparison between measured and predicted void fractions. 

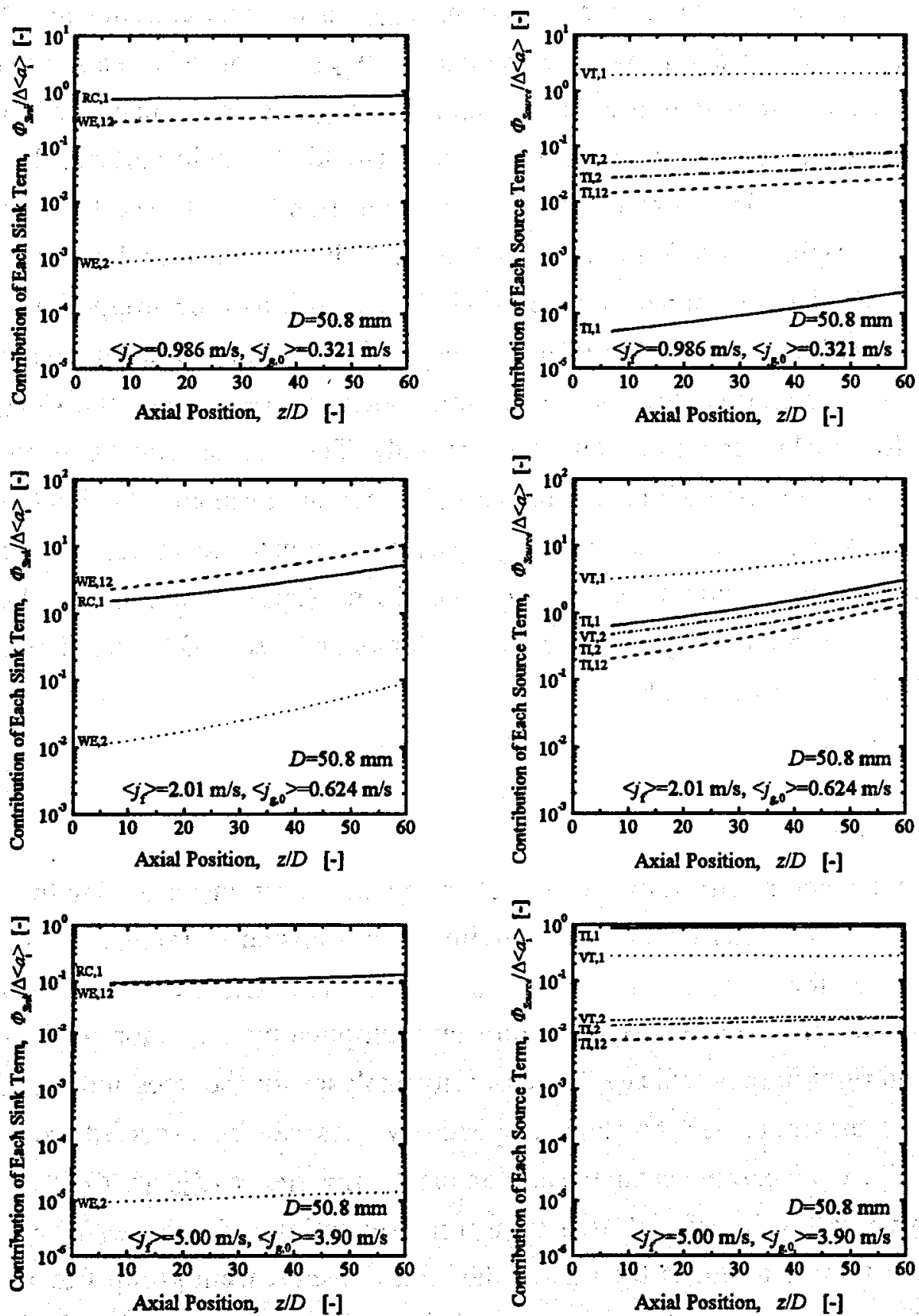

Figure 5.14. Scale analysis to determine the predominant terms among the source and sink terms. 
dividing the interfacial area change due to each sink or source term by $\left.\Delta<a_{\mathrm{i}}\right\rangle\left(=\left\langle a_{\mathrm{i}}\right\rangle-\left\langle a_{\mathrm{i} z} / D=6.00\right\rangle\right)$. The scale analysis for the sink term (left figures) shows that the coalescence due to bubble random collision, $\Phi_{\mathrm{RC}, 1}$, and wake entrainment, $\Phi_{\mathrm{WE}, 12}$, are predominant over that due to wake entrainment, $\Phi_{\mathrm{WE}, 2}$, for all of three flow conditions. Therefore, the sink term due to wake entrainment, $\Phi_{\mathrm{WE}, 2}$, could be neglected for the flow conditions tested in this study. However, this term would play an important role in the flow condition at the slug flow or at the slug-to-chum transition flow. The scale analysis for the source term (right figures) suggests that for relatively low liquid velocity such as $\left\langle j_{\mathrm{p}}\right\rangle=0.986 \mathrm{~m} / \mathrm{s}$, the predominant source term is the expansion of the group-I bubbles, $\Phi_{\mathrm{VT}, 1}$, because of small turbulence. As the liquid velocity increases, the breakup due to turbulent impact, $\Phi_{\mathrm{T}, 1}$, becomes significant. Other terms such as $\Phi_{\mathrm{VT}, 2}, \Phi_{\mathrm{T}, 12}$, and $\Phi_{\mathrm{T}, 2}$, could be neglected for the flow conditions tested in this study. These terms would play an important role in the flow condition with much higher void fraction of cap and slug bubbles.

Figure 5.15 shows the sensitivity analyses of adjustable valuables for three flow conditions such as $\left\langle j_{\mathrm{p}}\right\rangle=0.986 \mathrm{~m} / \mathrm{s}$ and $\left\langle j_{\mathrm{g}, 0}\right\rangle=0.321 \mathrm{~m} / \mathrm{s}$ (upper figures), $\langle j \mathrm{p}\rangle=2.01 \mathrm{~m} / \mathrm{s}$ and $\left\langle j_{\mathrm{g}, 0}\right\rangle=0.624 \mathrm{~m} / \mathrm{s}$ (middle figures), and $\langle j \mathrm{p}\rangle=5.00 \mathrm{~m} / \mathrm{s}$ and $\left\langle j_{\mathrm{g}, 0}\right\rangle=3.90 \mathrm{~m} / \mathrm{s}$ (lower figures). The sensitivity, $\Omega$, is defined by

$$
\Omega \equiv \frac{\left\langle a_{i}(\Lambda)\right\rangle}{\left\langle a_{i}(\Lambda=1)\right\rangle},
$$

where $\Lambda$ is the factor defined by the ratio of an adjustable parameter used in the sensitivity analysis to the parameter determined experimentally as listed in Table 5.5. For example, $\Lambda=\Gamma_{\mathrm{RC}, 1} \% .351$ for the sensitivity analysis of $\Gamma_{\mathrm{RC}, 1^{\circ}}$. In the sensitivity analysis, adjustable parameters except for the adjustable parameter multiplied by the factor are kept constant. The left and right figures indicate the sensitivity analyses for the rates and the efficiencies of bubble coalescence and breakup, respectively. As can be expected from the scale

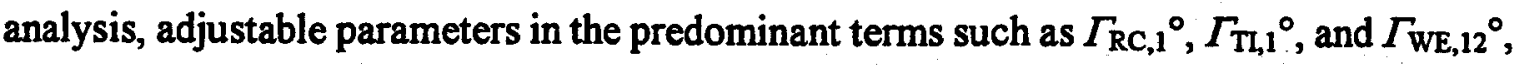
are sensitive to the interfacial area transport calculation. Since $\Gamma_{\mathrm{WE}, 12}{ }^{\circ}$ in the wake entrainment term determines the rate of the void transport from group I to II, it is very important parameter in the two-group interfacial area transport equations. On the other hand, experimental coefficients in the coalescence and breakup efficiencies would not be so sensitive for the flow condition where the void transport term is dominant over the interfacial area transport. As the contribution of bubble coalescence and breakup to the interfacial area transport comes to be large, the sensitivity would increase. For 

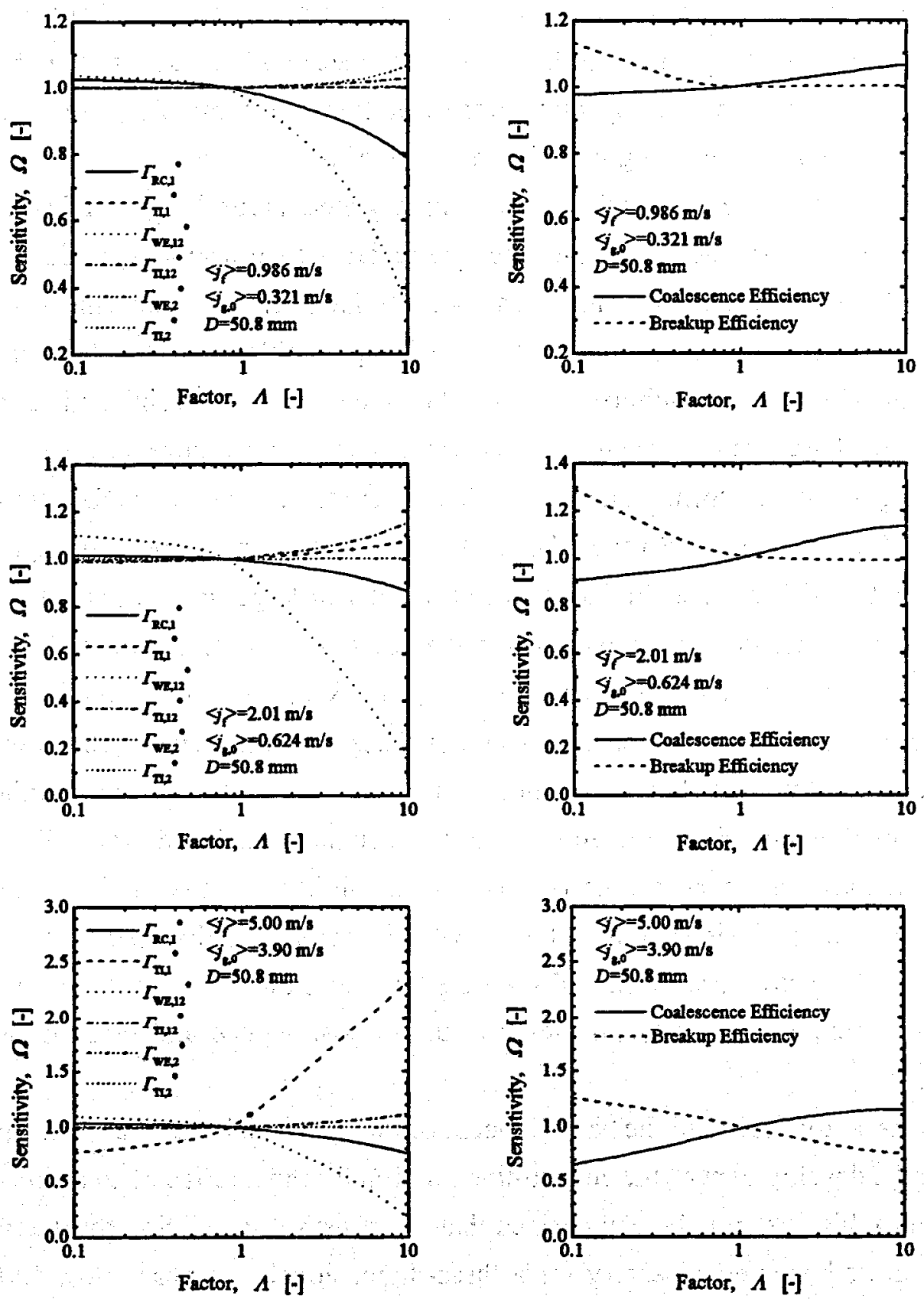

Figure 5.15. Sensitivity analysis of adjustable valuables in the two-group interfacial area transport equations. 
$\left\langle j_{\mathrm{f}}\right\rangle=0.986 \mathrm{~m} / \mathrm{s}$ and $\left\langle j_{\mathrm{g}, 0}\right\rangle=0.321 \mathrm{~m} / \mathrm{s}$, the void transport term, $\Phi_{\mathrm{VT}, 1}$ (see figure at the upper right in Fig.5.13) is dominant, resulting in low sensitivity of the experimental coefficients in the coalescence and breakup efficiencies to the interfacial area transport calculation. For $\left\langle j_{\mathrm{P}}\right\rangle=5.00 \mathrm{~m} / \mathrm{s}$ and $\left\langle j_{\mathrm{g}, 0}\right\rangle=3.90 \mathrm{~m} / \mathrm{s}$, the bubble coalescence and breakup terms such as $\Phi_{\mathrm{RC}, 1}$, $\Phi_{\mathrm{WE}, 12}$, and $\Phi_{\mathrm{TI}, 1}$, come to be comparable to the void transport term, $\Phi_{\mathrm{VT}, 1}$ (see figure at the upper right in Fig.5.13), resulting in high sensitivity of the coefficients.

This study demonstrates that the preliminary models for the sink and source terms are likely to be sound and the two-group interfacial area transport equations are very promising. However, in a large channel, another interfacial area transport mechanism such as the coalescence due to random collision of cap bubbles and the breakup due to interfacial instability of cap bubbles should be taken into account as sink and source terms in the interfacial area transport equation (see Table 4.1). Furthermore, in the future study, the modeling of the transformation of a bubble cluster into a cap bubble (Kalkach-Navarro et al., 1994; Millies et al., 1996) should be addressed. In addition to these, the applicability of the present model to other fluid systems, or smaller or larger diameter pipes should be examined experimentally, which means that the coefficients in this model, $\Gamma_{\mathrm{RC}, 1^{\circ}}, \Gamma_{\mathrm{T}, 1}{ }^{\circ}$,

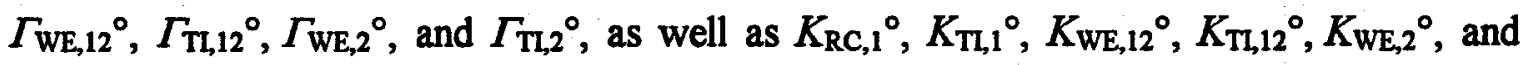
$K_{\mathrm{Th}, 2^{\circ}}$ should be modified based on a rigorous data base. In a future work, the flow fields of the gas and liquid-phases should be calculated by using a code and the interfacial area transport equation should be implemented in the computational code for calculating the local interfacial area concentration. The applicability of the derived models to three-dimensional analysis should be tested with rigorous local flow parameters taken at the bubbly-to-slug flow transition and the slug flow, and the effect of three-dimensional phase distribution and velocity profiles on bubble coalescence and breakup should be discussed.

This study described the basic concept of the interfacial area transport equation in relation to the development of the constitutive relation for the interfacial area concentration. In addition to this, it should be emphasized that the constitutive relation for the two-phase Reynolds stress becomes necessary for a three-dimensional two-phase flow formulation (Ishii, 1997b). As in the case of single phase flow, the Reynolds stress determines the transverse momentum transfer and velocity profile in the fluid. The simplest form of the constitutive relation is a two-phase mixing length model. A higher order turbulence model such as the $k-\varepsilon$ model (Lopez de Bertodano et al., 1994) is also possible. In the state-of-the-art of the two-phase flow modeling, there are almost no well-established or 
reliable turbulence models. Thus, as a long term goal, some efforts in this direction should be made for a three-dimensional model (Ishii, 1997b). The two-phase turbulence may be divided into two main categories: the flow shear-induced turbulence (macroscopic) and the interface-induced turbulence (microscopic). Turbulence is a non-linear phenomenon; however, as a first step, a linear combination of these two turbulence effects can be considered and modeled separately. 


\section{SUMMARY AND CONCLUSIONS}

In relation to the development of the interfacial area transport equation in two-phase flow systems, the development of local flow measurement techniques, the formulation of two-group interfacial area transport equations, the construction of database, the modeling of sink and source terms of interfacial area concentration were performed. As for the development of local flow measurement technique for gas phase, a miniaturized four-sensor conductivity probe and its signal processing scheme were developed. The sharp and highly conductive sensor tips, and miniaturized structure of the probe configuration could effectively minimize bubble deformation and missing bubble phenomena. Moreover, the new design accommodated the capability of a double-sensor probe for small bubbles, such that the two-phase parameters for both small and large bubbles could be obtained simultaneously. This was a significant improvement in view of building a database for the development of the two-group interfacial transport equation, because the new probe could be applied in flow conditions where different types of bubbles exist simultaneously. The four-sensor conductivity probe method was benchmarked by a well-designed experiment and a numerical simulation, its validity was demonstrated analytically and experimentally. The four-sensor conductivity probe system developed in this study at Purdue University was compared with the four-sensor optical probe system developed by French Atomic Energy Commission, France. It gave us some very useful information on the capabilities of both systems as well as on the main system components influencing the measurement accuracy.

As for the development of local flow measurement technique for liquid phase, hotfilm anemometer developed basically for single-phase flow was applied to two-phase flow. In order to apply the hotfilm anemometer to two-phase flow, a signal processing scheme was developed to remove signals due to passing bubbles from raw signals. The hotfilm probe method was benchmarked by a well-designed experiment and its validity was demonstrated experimentally.

As for the construction of database of local flow parameters, extensive experiments were performed basically by using $25.4-\mathrm{mm}$ and $50.8-\mathrm{mm}$ round tubes. The data from the multi-sensor conductivity probe gave near complete information on the 
time-averaged local hydrodynamic parameters of two-phase flow to model and evaluate sink and source terms of interfacial area concentration. Thus, the data set obtained in this study will eventually be used for the development of reliable constitutive relations, which reflect the true transfer mechanisms in bubbly flow systems.

As for the formulation of two-group interfacial area transport equations, a general approach to treat bubbles in two groups was proposed. The two-group interfacial area transport equations were formulated by considering two-groups of bubbles such as spherical/distorted bubbles (Group I) and cap/slug bubbles (Group II). Possible bubble coalescence and breakup patterns were classified into eight categories including the interchange terms between groups I and II.

As for the modeling of source and sink terms in the interfacial area transport equation, four mechanisms of bubble coalescence and breakup were considered: (1) the coalescence due to random collisions driven by turbulence, (2) the coalescence due to wake entrainment, (3) the breakup upon the impact of turbulent eddies, and (4) the breakup of large cap bubbles due to flow instability on the bubble surface. Based on the four basic mechanisms, the source and sink terms in the interfacial area transport equations were modeled. The one-group interfacial area transport equation could be applied to flow conditions for which the void fractions are lower than about $20 \%$. The mechanisms of bubble coalescence and breakup were successfully modeled by considering bubble random collision and impact of turbulent eddies. The interfacial area transport calculation by derived models suggested that the bubble interactions such as coalescence and breakup might not contribute to the interfacial area transport for relatively low liquid velocity and void fraction because of small bubble mean free path and breakup efficiency. As a consequence, the bubble expansion due to pressure reduction along the flow direction was dominant for the interfacial area transport. On the other hand, as the void fraction was increased, the contribution of the bubble random collision came to be significant for the interfacial area transport.

The two-group interfacial area transport equations were introduced to explain the interfacial area transport phenomena at the bubbly-to-slug flow transition. The preliminary model development for the bubble coalescence and breakup was conducted by considering bubble random collision, wake entrainment and turbulent impact. It turned out that the interchange term due to the turbulent impact on cap bubbles (turbulent impact) and the sink term in the group II due to the wake entrainment of cap bubbles played a minor role in the interfacial area transport in the tested flow conditions. This was explained by considering 
the small number of turbulent eddies available for the collision with cap bubbles, and the average distance between cap bubbles being much larger than the wake length effective for acceleration of cap bubbles.

The one-group interfacial area transport equation could predict interfacial area concentrations measured in a relatively low void fraction region within an average relative deviation of $\pm 9.51 \%$. The two-group interfacial area transport equations could give good predictions for the interfacial area concentration measured at the bubbly-to-slug flow transition within the average relative deviation of $\pm 3.61 \%$. It was demonstrated that the two-group interfacial area transport equations were promising for the prediction of the interfacial area transport in two-phase flow systems. 


\section{APPENDIX A. ORDER OF MAGNITUDE OF THE FREQUENCY OF BUBBLE RANDOM COLLISION}

Figure $\mathrm{Al}$ shows the frequency and rate of bubble random collision. Open and solid symbols indicate the calculated collision frequency between a single bubble and surrounding bubbles, $f_{\mathrm{RC}, 1}$, and the calculated collision rate (collision frequency per unit volume) between bubbles, $f_{\mathrm{RC}, 1}{ }^{\cdot} n_{\mathrm{b}, 1}$, respectively. The collision frequency and rate are calculated by Eq.(A1) and Eq.(A2), respectively.

$$
\begin{aligned}
& \left\langle f_{R C, 1}\right\rangle=\frac{\Gamma_{R C, 1}^{0}(\pi / 6)\left\langle\alpha_{1}\right\rangle\left\langle\varepsilon_{0}\right\rangle^{1 / 3}}{\left\langle D_{b, 1}\right\rangle^{2 / 3}\left(\left\langle\alpha_{R C, \text { max }}\right\rangle-\langle\alpha\rangle\right)}, \\
& \left\langle f_{R C, 1}\right\rangle \cdot\left\langle n_{b, 1}\right\rangle=\frac{\Gamma_{R C, 1}^{0}(\pi / 6)\left\langle\alpha_{1}\right\rangle^{2}\left\langle\varepsilon_{0}\right\rangle^{1 / 3}}{\left\langle D_{b, 1}\right\rangle^{11 / 3}\left(\left\langle\alpha_{R C, \text { max }}\right\rangle-\langle\alpha\rangle\right)} .
\end{aligned}
$$

As can be seen from the forms of the above equations, the calculated collision frequency and rate gradually increase with the increase of the void fraction and the liquid velocity. Magnitudes of the collision frequency for $\langle j p=0.491,0.986,2.01$, and $5.00 \mathrm{~m} / \mathrm{s}$ range from $6 \times 10^{-3}$ to $5 \times 10^{-2} \mathrm{~s}^{-1}$, from $1 \times 10^{-2}$ to $8 \times 10^{-2} \mathrm{~s}^{-1}$, from $2 \times 10^{-2}$ to $1 \times 10^{-1} \mathrm{~s}^{-1}$, and from $7 \times 10^{-2}$ to $1 \mathrm{~s}^{-1}$, respectively. On the other hand, magnitudes of the collision rate for $\left\langle j_{\mathrm{p}}\right\rangle=0.491$, $0.986,2.01$, and $5.00 \mathrm{~m} / \mathrm{s}$ range from $2 \times 10^{4}$ to $5 \times 10^{5} \mathrm{~m}^{-3} \mathrm{~s}^{-1}$, from $5 \times 10^{4}$ to $9 \times 10^{5} \mathrm{~m}^{-3} \mathrm{~s}^{-1}$, from $1 \times 10^{5}$ to $1 \times 10^{6} \mathrm{~m}^{-3} \mathrm{~s}^{-1}$, and from $2 \times 10^{6}$ to $6 \times 10^{7} \mathrm{~m}^{-3} \mathrm{~s}^{-1}$, respectively. It should be noted here that the collision frequency between a single eddy and surrounding bubbles, $\left\langle f_{T, 1}\right\rangle$, is about the same order of the collision frequency between a single bubble and surrounding bubbles, $\left\langle f_{\mathrm{RC}, 1}\right\rangle$. 


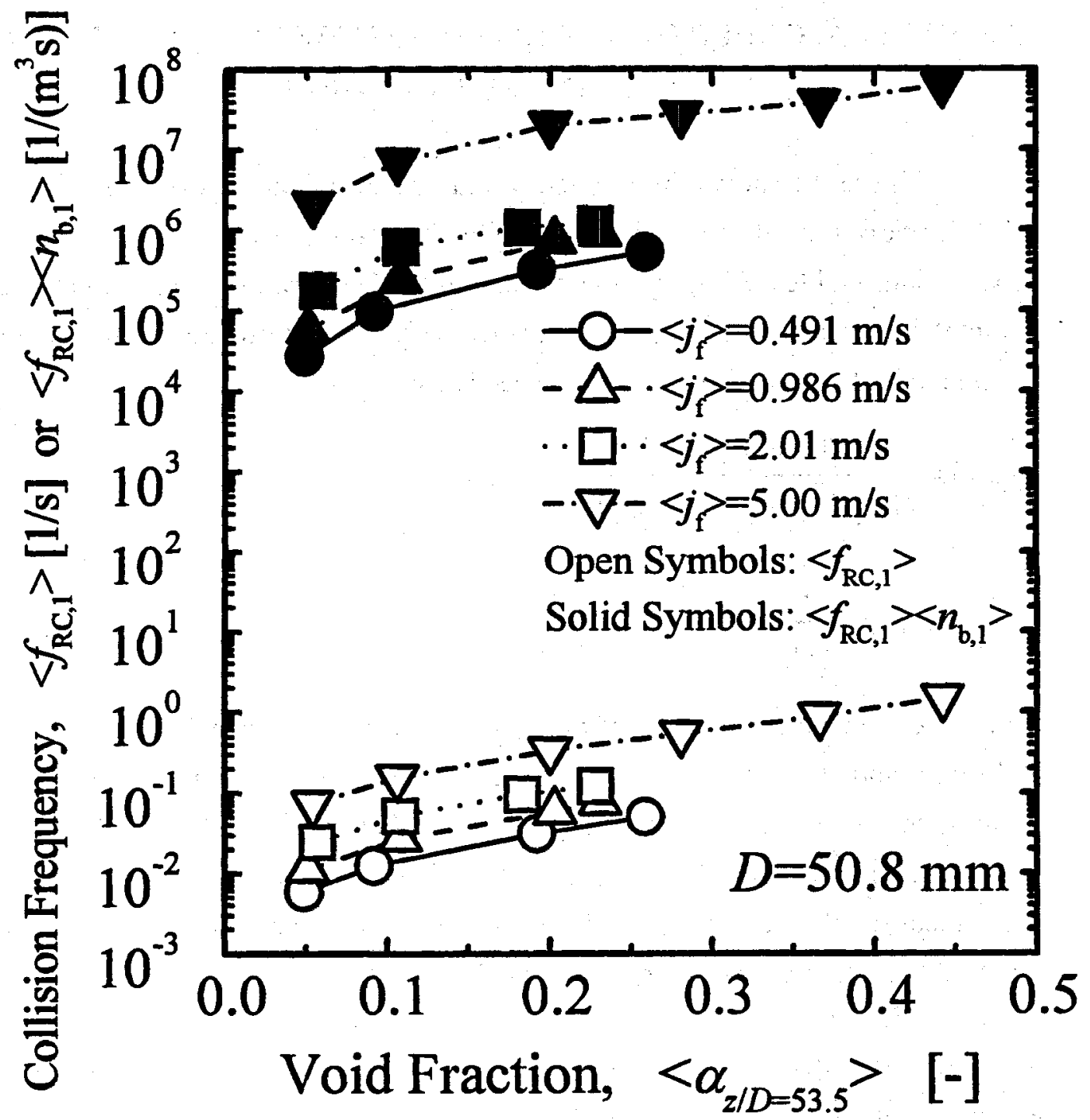

Figure A.1.1 Order of magnitude of the frequency of bubble random collision. 


\section{LIST OF REFERENCES}

Azbel, D., Athanasios, I. L., 1983. A mechanism of liquid entrainment, Handbook of Fluids in Motion, Ann Arbor Science Publishers, Ann Arbor, USA.

Bilicki, A., Kestin, J., 1987. Transition criteria for two-phase flow patterns in vertical upward flow, International Journal of Multiphase Flow 13, 283-294.

Chesters, A. K., Hoffman, G., 1982. Bubble coalescence in pure liquids, Applied Scientific Research 38, 353-361.

Chisholm, D. 1967. A theoretical basis for the Lockhart-Martinelli correlation for two-phase flow, International Journal of Heat and Mass Transfer 10, 1767-1778.

Clift, R., Grace, J. R., Weber, M. E., 1978. Bubbles, Drops, and Particles, Academic Press, New York, USA.

Coulaloglou, C. A., and Tavlarides, L. L., 1977. Description of interaction processes in agitated liquid-liquid dispersions, Chemical Engineering Science 32, 1289-1297.

Drew, D. A., Passman, S. L., 1998. Theory of Multicomponent Fluids, Applied Mathematical Sciences 135, Springer, New York, USA.

Grossetete, C., 1995. Experimental investigation and preliminary numerical simulations of void profile development, Proceedings of 2nd International Conference on Multiphase Flow 2, pp.IF1-1-IF1-10.

Hibiki, T., Ishii, M., 1997. Interfacial are and interfacial transfer in two-phase flow systems, Technical Report, PU-NE-97/7, School of Nuclear Engineering, Purdue University, West Lafayette, IN, USA.

Hibiki, T., Hogsett, S., Ishii, M., 1998a. Local measurement of interfacial area, interfacial velocity and liquid turbulence in two-phase flow, Nuclear Engineering and Design 184, 287-304.

Hibiki, T., Ishii, M., Xiao, Z., 1998b. Local flow measurements of vertical upward air-water flow in a round tube, Proceedings of 3rd International Conference on Multiphase Flow, Paper Number-210.

Hibiki, T., Ishii, M., 1999a. Experimental study on interfacial area transport in bubbly two-phase flows, International Journal of Heat and Mass Transfer 42; 3019-3035.

Hibiki, T., and Ishii, M., 1999b. Interfacial area transport of adiabatic air-water bubbly 
flow in verticnl round tubes, Proceedings of 33rd National Heat Transfer Conference HTD99-51.

Hibiki, T., Ishii, M., 2000a. One-group interfacial area transport of bubbly flows in vertical round tubes, International Journal of Heat and Mass Transfer 43 (2000) 2711-2726.

Hibiki, Ishii, M., 2000b. Two-group interfacial area transport equations at bubbly-to-slug flow transition, Nuclear Engineering and Design 202, 39-76.

Hibiki, Ishii, M., Xiao, Z., 2001. "Axial Interfacial Area Transport of Vertical Bubbly Flows," International Journal of Heat and Mass Transfer 44, 1869-1888.

Ishii, M., 1975. Thermo-Fluid Dynamic Theory of Two-Phase Flow, Eyrolles, Paris.

Ishii, M., 1977. One-dimensional drift-flux model and constitutive equations for relative motion between phases in various two-phase flow regimes, Technical report ANL-77-47, Argonne National Laboratory, Chicago, II, USA.

Ishii, M., 1997a. Interfacial area measurement and interfacial area transport equation, Proceedings of 1997. ASME Fluids Engineering Division Summer Meeting, FEDSM97-3527.

Ishii, M., 1997b. Views on the future of thermal hydraulic modeling, Proceedings of the OECD/CSNI Workshop on Transient Thermal-Hydraulic and Neutronic Codes Requirements, NUREG/CP-0159, pp.751-759.

Ishii, M., Chawla, T. C., 1979. Local drag laws in dispersed two-phase flow, Technical report ANL-79-105, Argonne National Laboratory, Chicago, II, USA.

Ishii, M., Kojasoy, G., 1993. Interfacial area transport equation and preliminary considerations for closure relations, Technical Report, PU-NE-93/6, School of Nuclear Engineering, Purdue University, West Lafayette, IN, USA.

Ishii, M., Wu, Q., Revankar, S. T., Hibiki, T., Leung, W. H., Hogsett, S., Kashyap, A., 1997. Interfacial area transport in two-phase bubbly flow, Proceedings of 15th Symposium of Energy Engineering Science.

Ishii, M., Wu, Q., Assad, A., Uhle, J. 1998. Interfacial area transport equation for two-fluid model formulation, Proceedings of IMuST Meeting.

Ishii, M., Zuber, N., 1979. Drag coefficient and relative velocity in bubbly, droplet or particulate flows, AIChE Journal 25, 843-855.

Kalkach-Navarro, S., Lahey, Jr., R. T., Drew, D. A., 1994. Analysis of the bubbly/slug flow regime transition, Nuclear Engineering and Design 151, 15-39.

Kashyap, A., Ishii, M., Revankar, S. T., 1994. An experimental and numerical analysis of structural development of two-phase flow in a pipe, Technical Report, PU-NE-94/2, 
School of Nuclear Engineering, Purdue University, West Lafayette, IN, USA.

Kataoka, I., Ishii, M., Serizawa, A., 1986. Local formulation and measurements of interfacial area concentration in two-phase flow. Internationall Journal of Multiphase flow 12, 505-529.

Kataoka, I., Ishii, M., Serizawa, A., 1994. Sensitivity analysis of bubble size and probe geometry on the measurements of interfacial area concentration in gas-liquid two-phase flow. Nuclear Engineering and Design 146, 53-70.

Kataoka, I., Serizawa, A., 1990. Interfacial area concentration in bubbly flow, Nuclear Engineering and Design 120, 163-180.

Kim, S., Fu, X. Y., Wang, X., Ishii, M., 1998. Local interfacial area concentration measurement in a two-phase flow using a four-sensor conductivity probe, Transactions of ANS 79, 356-357.

Kim, S., Wang, X., Fu, X. Y., Ishii, M., 1999. Development of noble four-sensor conductivity probe and its signal processing scheme, Proceedings of 7 th International Conference on Nuclear Engineering, ICONE-7196.

Kim, S., Fu, X. Y., Wang, X., Ishii, M., 2000. Development of the miniaturized four-sensor conductivity probe and the signal processing scheme, International Journal of Heat and Mass Transfer 43, 4101-4118.

Kocamustafaogullari, G., Ishii, M., 1985. Maximum fluid particle size for bubbles and drops, Proceedings of ASME Winter Annual Meeting FED-Vol.29, pp.99-107.

Kocamustafaogullari, G., Huang, W. D., Razi, J., 1994. Measurement of modeling of average void fraction, bubble size and interfacial area, Nuclear Engineering and Design 148, 437-453.

Kocamustafaogullari, G., Ishii, M., 1983. Interfacial area and nucleation site density in boiling systems, International Journal of Heat and Mass Transfer 26, 1377-1387.

Kocamustafaogullari, G., and Ishii, M., 1995. Foundation of the interfacial area transport equation and its closure relations, International Journal of Heat and Mass Transfer 38, $481-493$.

Lafi, A. Y., and Reyes, Jr, J. N., 1991. Phenomenological models for fluid particle coalescence and breakage, Technical Report, OSU-NE-9120, Department of Nuclear Engineering, Oregon State University, Corvallis, OR, USA.

Le Corre, J, M., Fu, X. Y., Ishii, M., 2000. Conductivity and optical four-sensor probe benchmark, PU-NE-00/21, School of Nuclear Engineering, Purdue University, West Lafayette, IN, USA. 
Le Corre, J. M., Ishii, M., 2002. Numerical evaluation and correction method for multi-sensor probe measurement techniques in two-phase bubbly flow, Nuclear Engineering and Design 216, 221-238.

Leung, W. H., Eberle, C. S., Wu, Q., Ueno, T., Ishii, M., 1995. Quantitative characterizations of phasic structure developments by local measurement methods in two-phase flow, Proceedings of 2nd International Conference on Multiphase Flow 1, pp.IN-2-IN-17.

Lockhart, R. W., Martinelli, R. C., 1949. Proposed correlation $f$ data for isothermal two-phase, two-component flow in pipes, Chemical Engineering Progress 5, 39-48.

Loeb, L. B., 1927. The Kinetic Theory of Gases, Dover, New York, USA.

Lopez de Bertodano, M., Lahey, Jr., R.T., Jones, O. C., 1994. Development of $k-\varepsilon$ model for bubbly two-phase flow, Transactions of the ASME 116, 128-134.

Millies, M., Drew, D. A., Lahey, Jr., R. T., 1996. A first order relaxation model for the prediction of the local interfacial area density in two-phase flows, International Journal of Multiphase Flow 22, 1073-1104.

Millies, M. Mewes D., 1995. A transport equation for the local interfacial area density in two-phase flows, Proceedings of 2nd International Conference on Multiphase Flow 3, pp.MO3-7-MO3-13.

Millies, M., Mewes, D., 1999. Interfacial area density in bubbly flow, Chemical Engineering and Processing 38, 307-319.

Moore, W. J., 1972. Physical Chemistry, Prentice-Hall, Inc., New Jersey, USA.

Morel, C., Goreaud, N., Delhaye, J. M., 1999. The local volumetric interfacial area transport equation: derivation and physical significance, International Journal of Multiphase Flow 25, 1099-1128.

Morita, G., Nakahara, Y., Makino, Y., Tomiyama, A., 1999. Study on terminal rising velocity and shape of single bubbles moving in a vertical pipe, Proceedings of 18th Multiphase Flow Symposium '99, pp.7-8.

Oolman, T., Blanch, H. W., 1986a. Bubble coalescence in air-sparged bioreactors, Biotechnique Bioengineering 28, 578-584.

Oolman, T., Blanch, H. W., 1986b. Bubble coalescence in stagnant liquids, Chemical Engineering Communication 43, 237-261.

Otake, T., Tone, S., Nakao, K., Mitsuhashi, Y., 1977. Coalescence and breakup of bubbles in liquids, Chemical Engineering Science 32, 377-383.

Prince, M. J., and Blanch, H. W., 1990. Bubble coalescence and break-up in air-sparged 
bubble columns, AIChE Journal 36, 1485-1497.

Revankar, S. T., Ishii, M., 1992. Local interfacial area measurement in bubbly flow, International Journal of Heat and Mass Transfer 35, 913-925.

Revankar, S. T., Ishii, M., 1993. Theory and measurement of local interfacial area using a four sensor probe in two-phase flow, International Journal of Heat and Mass Transfer 36, 2997-3007.

Reyes, J. N., 1989 Statistically derived conservation equations for fluid particle flows, Proceedings of ANS Winter Meeting, pp.669-670.

Riznic, J. R., Ishii, M., 1989. Bubble number density and vapor generation in flashing flow 32, 1821-1833.

Schlichting, H., 1979. Boundary-layer Theory, McGraw-Hill, New York, USA.

Serizawa, A., Kataoka, I., 1988. Phase distribution in two-phase flow, Transient Phenomena in Multiphase Flow, Hemisphere, Washington, USA, pp.179-224.

Städtke, H., Blahak, A., Worth, B., 1997. Modelling of transport of interfacial area concentration in two-phase flow systems, Proceedings of 8th International Topical Meeting on Nuclear Reactor Thermal-Hydraulics 1, 69-78.

Stewart, C. W., 1995. Buble interaction in low-viscosity liquids, International Journal of Multiphase Flow 21, 1037-1046.

Taitel, Y., Bornea, D., Dukler, A. E., 1980. Modelling flow pattern transitions for steady upward gas-liquid flow in vertical tubes, AIChE Journal 26, 345-354.

Tomiyama, A., Kataoka, I., Sakaguchi, T., 1995. Drag coefficients of bubbles ( $1^{\text {st }}$ report, drag coefficients of a single bubble in a stagnant liquid), Transactions of JSME 61, 2357-2364.

Tsouris, C., and Tavlarides, L. L., 1994. Breakage and coalescence models for drops in turbulent dispersions, AIChE Journal 40, 395-406.

Tsuchiya, K., Miyahara, T., Fan, L. S., 1989. Visualization of bubble-wake interactions for a stream of bubbles in a two-dimensional liquid-solid fluidized bed, International Journal of Multiphase Flow 15, 35-49.

Uhle, J., Wu, Q., Ishii, M., 1998. Dynamic flow regime modeling, Proceedings of 6th International Conference on Nuclear Engineering, ICONE-6509.

Wu, Q., Kim, S., Ishii, M., Beus, S. G., 1997a. One-group interfacial area transport in vertical bubbly flow, Proceedings of 32nd National Heat Transfer Conference HTC-10, 67-79.

Wu, Q., Kim, S., McCreary, D., Ishii, M., Beus, S. G., 1997b. Measurement of interfacial 
area concentration in two-phase bubbly flow, ANS Transaction 78, 437.

Wu, Q., Ishii, M., 1999. Sensitivity study on double-sensor conductivity probe for the measurement of interfacial area concentration in bubbly flow, International Journal of Multiphase Flow 25, 155-173.

Wu, Q., Ishii, M., Uhle, J., 1998a. Framework of two-group model for interfacial area transport in vertical two-phase flows, Transactions of ANS 79, pp.351-352.

Wu, Q., Kim, S., Ishii, M., Beus, S. G., 1998b. One-group interfacial area transport in vertical bubbly flow", International Journal of Heat and Mass Transfer 41, 1103-1112. 


\section{LIST OF JOURNAL PUBLICATIONS RELATED TO DEVELOPMENT OF INTERFACIAL AREA TRANSPORT EQUATION PERFORMED AT THERMAL-HYDRAULICS AND REACTOR SAFETY LABORATORY IN PURDUE UNIVERISTY}

1. Revankar, S. T., Ishii, M. 1992. "Local Interfacial Area Measurement in Bubbly Flow," International Journal of Heat and Mass Transfer 35, 913-925.

2. Revankar, S. T., Ishii, M. 1993. "Local Interfacial Area Using Four Sensor Probe in Two-phase Flow," International Journal of Heat and Mass Transfer 36, 2997-3007.

3. Kataoka, I., Ishii, M., Serizawa, A. 1994. "Sensitivity Analysis of Bubble Size and Probe Geometry on the Measurements of Interfacial Area Concentration in Gas-Liquid Two-phase Flow," Nuclear Engineering and Design 146, 53-70.

4. Leung, W. H., Revankar, S. T., Ishii, Y., Ishii, M., 1995. “Axial Development of Interfacial Area and Void Concentration Profiles Measured by Double-Sensor Probe Method," International Journal of Heat and Mass Transfer 38, 445-453.

5. Kocamustafaogullari, G., Ishii, M., 1995. "Foundation of Interfacial Area Transport Equation and Its Closure Relations," International Journal of Heat and Mass Transfer 38, $481-493$.

6. Hogsett, S., Ishii, M., 1997. "Local Two-phase Flow Measurements Using Sensor Techniques," Nuclear Engineering and Design 175, 15-24.

7. Wilmarth, T., Ishii, M., 1997. "Interfacial Area Concentration and Void Fraction in Narrow Rectangular Vertical Channel," Journal of Fluid Engineering 119, 916-922. 
8. Wu, Q., Kim, S., Ishii M., Beus, S. G., 1998. “One-group Interfacial Area Transport in Vertical Bubbly Flow," International Journal of Heat and Mass Transfer 41, 1103-1112.

9. Hibiki, T., Hogsett, S., Ishii, M., 1998. "Local Measurement of Interfacial Area, Interfacial Velocity and Liquid Turbulence in Two-phase flow," Nuclear Engineering and Design 184, 287-304.

10. Wu, Q., Ishii, M., 1999. "Sensitivity Study on Double-sensor Conductivity Probe for the Measurement of Interfacial Area Concentration," International Journal of Multiphase Flow 25, 155-173.

11. Hibiki, T., Ishii, M., 1999. "Experimental Study on Interfacial Area Transport in Bubbly Two-Phase Flow," International Journal of Heat and Mass Transfer 42, 3019-3035.

12. Kim, S., Fu, X. Y., Wang, X., Ishii, M., 2000. "Development of the Miniaturized Four-Sensor Conductivity Probe and the Signal Processing Scheme," International Journal of Heat and Mass Transfer 43, 4101-4118.

13. Hibiki, T., Ishii, M., 2000. "One-Group Interfacial Area Transport of Bubbly Flows in Vertical Round Tubes," International Journal Heat and Mass Transfer 43, 2711-2726.

14. Hibiki, T., Ishii, M., 2000. "Two-Group Interfacial Area Transport Equations at Bubbly-to-Slug Flow Transition," Nuclear Engineering and Design 202, 39-76.

15. Hibiki, T., Ishii, M., Xiao, Z., 2001. "Axial Interfacial Transport of Vertical Bubbly Flows," International Journal of Heat and Mass Transfer 44, 1869-1888.

16. Hibiki, T., Ishii, M., 2001. "Interfacial Area Concentration in Steady Fully Developed Bubbly Flows," International Journal of Heat and Mass Transfer 44, 3443-3461. 
17. Ishii, M., Kim, S., 2001. "Micro Four-Sensor Probe Measurement of Interfacial Area Transport for Bubbly Flow in Round Pipes," Nuclear Engineering and Design 205, 123-131.

18. Kim, S., Fu, X. Y., Wang, X., Ishii, M., 2001. "Study on Interfacial Structures in Slug Flows Using a Miniaturized Four-Sensor Conductivity Probe," Nuclear Engineering and Design 204, 45-55.

19. Bartel, M. D., Ishii, M., Masukawa, T., Mi, Y., Situ, R., 2001. "Interfacial Area Measurements in Subcooled Flow Boiling," Nuclear Engineering and Design 210, 135-155.

20. Hibiki, T. Takamasa, T., Ishii, M., 2001. "Interfacial Area Transport of Bubbly Flow in a Small Diameter Pipe," Journal of Nuclear Science and Technology 38, 614-620.

21. Hibiki, T., Ishii, M., 2002. "Development of One-group Interfacial Area Transport Equation in Bubbly Flow Systems, "International Journal of Heat and Mass Transfer 45, 2351-2372.

22. Ishii, M., Kim, S., Uhle, J., 2002. "Interfacial Area Transport Equation: Model Development and Benchmark Experiments," International Journal of Heat and Mass Transfer 45, 3113-3123.

23. LaCorre, J. M., Ishii, M., 2002. "Numerical Evaluation and Correction Method for Multi-sensor Probe Measurement Techniques in Two-phase Bubbly Flow," Nuclear Engineering and Design 216, 221-238. 